TRANSACTIONS OF THE

AMERICAN MATHEMATICAL SOCIETY

Volume 356, Number 9 , Pages 3687-3720

S 0002-9947(04)03420-8

Article electronically published on January 13, 2004

\title{
IDEALS IN A PERFECT CLOSURE, LINEAR GROWTH OF PRIMARY DECOMPOSITIONS, AND TIGHT CLOSURE
}

\author{
RODNEY Y. SHARP AND NICOLE NOSSEM
}

\begin{abstract}
This paper is concerned with tight closure in a commutative Noetherian ring $R$ of prime characteristic $p$, and is motivated by an argument of K. E. Smith and I. Swanson that shows that, if the sequence of Frobenius powers of a proper ideal $\mathfrak{a}$ of $R$ has linear growth of primary decompositions, then tight closure (of $\mathfrak{a}$ ) 'commutes with localization at the powers of a single element'. It is shown in this paper that, provided $R$ has a weak test element, linear growth of primary decompositions for other sequences of ideals of $R$ that approximate, in a certain sense, the sequence of Frobenius powers of $\mathfrak{a}$ would not only be just as good in this context, but, in the presence of a certain additional finiteness property, would actually imply that tight closure (of a) commutes with localization at an arbitrary multiplicatively closed subset of $R$.

Work of M. Katzman on the localization problem for tight closure raised the question as to whether the union of the associated primes of the tight closures of the Frobenius powers of $\mathfrak{a}$ has only finitely many maximal members. This paper develops, through a careful analysis of the ideal theory of the perfect closure of $R$, strategies for showing that tight closure (of a specified ideal $\mathfrak{a}$ of $R$ ) commutes with localization at an arbitrary multiplicatively closed subset of $R$ and for showing that the union of the associated primes of the tight closures of the Frobenius powers of $\mathfrak{a}$ is actually a finite set. Several applications of the strategies are presented; in most of them it was already known that tight closure commutes with localization, but the resulting affirmative answers to Katzman's question in the various situations considered are believed to be new.
\end{abstract}

\section{INTRODUCTION}

This paper was motivated by a desire to explore the property, which might be possessed by certain sequences of proper ideals in a commutative Noetherian ring of prime characteristic, of having linear growth of primary decompositions. The property has been studied by K. E. Smith and I. Swanson [15] in the context of the localization problem for tight closure.

Received by the editors January 9, 2003 and, in revised form, May 15, 2003.

2000 Mathematics Subject Classification. Primary 13A35, 13E05, 13A15; Secondary 13B02, 13H05, 13F40, 13J10, 16S34, 16S36.

Key words and phrases. Commutative Noetherian ring, prime characteristic, Frobenius homomorphism, perfect closure, tight closure, plus closure, (weak) test element, primary decomposition, linear growth of primary decompositions, associated prime ideals, skew polynomial ring, skew Laurent polynomial ring, regular ring, pure subring, excellent rings.

The first author was partially supported by the Engineering and Physical Sciences Research Council of the United Kingdom (Overseas Travel Grant Number GR/S11459/01) and the Mathematical Sciences Research Institute, Berkeley.

The second author was supported by a fees-only studentship provided by the Engineering and Physical Sciences Research Council of the United Kingdom. 
To set the scene, let $R$ be a commutative Noetherian ring of prime characteristic $p$, and let $\mathfrak{a}$ be a proper ideal of $R$. For $n \in \mathbb{N}_{0}$ (we use $\mathbb{N}_{0}$ (respectively $\mathbb{N}$ ) to denote the set of non-negative (respectively positive) integers), the $n$-th Frobenius power $\mathfrak{a}^{\left[p^{n}\right]}$ of $\mathfrak{a}$ is the ideal of $R$ generated by all $p^{n}$-th powers of elements of $\mathfrak{a}$. Also, $R^{\circ}$ denotes the complement in $R$ of the union of the minimal prime ideals of $R$.

An element $r \in R$ belongs to the tight closure $\mathfrak{a}^{*}$ of $\mathfrak{a}$ if and only if there exists $c \in R^{\circ}$ such that $c r^{p^{n}} \in \mathfrak{a}^{\left[p^{n}\right]}$ for all $n \gg 0$. The theory of tight closure was invented by M. Hochster and C. Huneke [3], and many applications have been found for the theory; see [7. A major open problem about this theory concerns the question of whether tight closure 'commutes with localization'; see [7] Chapter 12]. It is known that it does for many particular choices of $R$ and $\mathfrak{a}$; see Aberbach-Hochster-Huneke [1], and also [7, Chapter 12].

The starting point for the work in this paper is the following: if the Frobenius powers of the proper ideal $\mathfrak{a}$ of $R$ have linear growth of primary decompositions in the sense that there exists a positive integer $h$ such that, for every non-negative integer $n$, there exists a minimal primary decomposition $\mathfrak{a}^{\left[p^{n}\right]}=\mathfrak{q}_{1, n} \cap \cdots \cap \mathfrak{q}_{k_{n}, n}$ with $\sqrt{\mathfrak{q}_{i, n}}\left[p^{n}\right] h \subseteq \mathfrak{q}_{i, n}$ for all $i=1, \ldots, k_{n}$, then tight closure of $\mathfrak{a}$ commutes with localization at a multiplicatively closed subset consisting of the powers of a single element of $R$, that is, $\mathfrak{a}^{*} R_{u}=\left(\mathfrak{a} R_{u}\right)^{*}$ for all $u \in R$. A proof of this fact can be extracted from the proof of [15, Corollary (1.3)].

It should be noted that Swanson established an analogous linear growth property for the ordinary powers of a proper ideal in an arbitrary commutative Noetherian ring in [16, Theorem 3.4]. The present first author subsequently provided a shorter proof of a more general result in [13.

In this paper, we shall approach questions about linear growth of primary decompositions by studying the ideals in the perfect closure $R^{\infty}$ of $R$. When $R$ is not reduced, by the perfect closure of $R$ we mean the perfect closure of $R_{\text {red }}:=R / \sqrt{(0)}$ : we recall M. J. Greenberg's work [2] in this context in \$1.

A study of the ideals of $R^{\infty}$ leads us to the concept of an $f$-sequence of ideals of $R$, that is, a sequence $\left(\mathfrak{a}_{n}\right)_{n \in \mathbb{N}_{0}}$ of ideals of $R$ such that $f^{-1}\left(\mathfrak{a}_{n+1}\right)=\mathfrak{a}_{n}$ for all $n \in \mathbb{N}_{0}$, where $f: R \longrightarrow R$ denotes the Frobenius homomorphism. The sequence $\left(\left(\mathfrak{a}^{\left[p^{n}\right]}\right)^{*}\right)_{n \in \mathbb{N}_{0}}$ of tight closures of the Frobenius powers of the ideal $\mathfrak{a}$ of $R$ is one example of an $f$-sequence. Another comes from taking the $F$-closures of the Frobenius powers of $\mathfrak{a}$ : the $F$-closure $\mathfrak{a}^{F}$ of $\mathfrak{a}$ is defined as

$$
\mathfrak{a}^{F}:=\left\{r \in R: \text { there exists } n \in \mathbb{N}_{0} \text { such that } r^{p^{n}} \in \mathfrak{a}^{\left[p^{n}\right]}\right\},
$$

and $\left(\left(\mathfrak{a}^{\left[p^{n}\right]}\right)^{F}\right)_{n \in \mathbb{N}_{0}}$ is another example of an $f$-sequence. A third example involves the so-called plus closures in the case when $R$ is a domain: the plus closure $\mathfrak{a}^{+}$of $\mathfrak{a}$ is defined to be the contraction back to $R$ of the extension of $\mathfrak{a}$ to the integral closure of $R$ in an algebraic closure of its field of fractions, and we shall see in $\$ 5$ that $\left(\left(\mathfrak{a}^{\left[p^{n}\right]}\right)^{+}\right)_{n \in \mathbb{N}_{0}}$ is an $f$-sequence.

It turns out that the set of $f$-sequences of ideals of $R$ is in a natural bijective correspondence with the set of ideals of the perfect closure $R^{\infty}$ of $R$. We explore this bijective correspondence in some detail in the early part (\$3 \$4 and 96 ) of this paper, building on work of D. A. Jordan [9] that provides, among other things, a rather concrete description of $R^{\infty}$. 
The sequence of Frobenius powers of an ideal $\mathfrak{a}$ of $R$ is not always an $f$-sequence. In $\S 7$ of the paper, we extend the concept of linear growth of primary decompositions to $f$-sequences in the obvious way. One of the main results of that section is Theorem 7.9, which shows that if a proper ideal of $R^{\infty}$ has a primary decomposition, then the $f$-sequence $\left(\mathfrak{a}_{n}\right)_{n \in \mathbb{N}_{0}}$ of ideals of $R$ to which it corresponds has linear growth of primary decompositions, and $\bigcup_{n \in \mathbb{N}_{0}}$ ass $\mathfrak{a}_{n}$ is finite. It should be noted that $R^{\infty}$ is only Noetherian in rather uninteresting situations, so that the existence of a primary decomposition for a proper ideal of $R^{\infty}$ would be a bonus that we should not expect in all cases.

Several of the main results of this paper involve the hypothesis that $R$ has a $p^{m_{0}}$-weak test element for some $m_{0} \in \mathbb{N}_{0}$. A $p^{m_{0}}$-weak test element is an element $c^{\prime} \in R^{\circ}$ such that, for every ideal $\mathfrak{b}$ of $R$ and for $r \in R$, it is the case that $r \in \mathfrak{b}^{*}$ if and only if $c^{\prime} r^{p^{n}} \in \mathfrak{b}^{\left[p^{n}\right]}$ for all $n \geq m_{0}$. A $p^{0}$-weak test element is called a test element. It is a result of Hochster and Huneke [5. Theorem (6.1)(b)] that an algebra of finite type over an excellent local ring of characteristic $p$ has a $p^{m_{0}}$-weak test element for some $m_{0} \in \mathbb{N}_{0}$.

In Theorem [7.6 we relate linear growth of primary decompositions for certain $f$-sequences to the general localization problem for tight closure, on the assumption that $R$ has a weak test element. This result, when used in conjunction with the above-mentioned Theorem 7.9 has the following consequence: if $R$ has a $p^{m_{0}}$-weak test element, $\mathfrak{a}$ is a proper ideal of $R$ and $\left(\mathfrak{a}_{n}\right)_{n \in \mathbb{N}_{0}}$ is an $f$-sequence of ideals of $R$ that approximates the Frobenius powers of $\mathfrak{a}$ in the sense that $\mathfrak{a}^{\left[p^{n}\right]} \subseteq \mathfrak{a}_{n} \subseteq$ $\left(\mathfrak{a}^{\left[p^{n}\right]}\right)^{*}$ for each $n \in \mathbb{N}_{0}$, and if the ideal of $R^{\infty}$ to which the $f$-sequence $\left(\mathfrak{a}_{n}\right)_{n \in \mathbb{N}_{0}}$ corresponds has a primary decomposition, then $\mathfrak{a}^{*} S^{-1} R=\left(\mathfrak{a} S^{-1} R\right)^{*}$ for every multiplicatively closed subset $S$ of $R$. Furthermore, we show in Theorem 7.13 that, in these circumstances, the ideal of $R^{\infty}$ to which the $f$-sequence $\left(\left(\mathfrak{a}^{\left[p^{n}\right]}\right)^{*}\right)_{n \in \mathbb{N}_{0}}$ corresponds also has a primary decomposition, so that $\left(\left(\mathfrak{a}^{\left[p^{n}\right]}\right)^{*}\right)_{n \in \mathbb{N}_{0}}$ has linear growth of primary decompositions and the set $\bigcup_{n \in \mathbb{N}_{0}} \operatorname{ass}\left(\mathfrak{a}^{\left[p^{n}\right]}\right)^{*}$ is finite. This is of interest because M. Katzman's approach in [10] to the localization problem for tight closure led to the following question: is it always the case that $\bigcup_{n \in \mathbb{N}_{0}} \operatorname{ass}\left(\mathfrak{a}^{\left[p^{n}\right]}\right)^{*}$ has only finitely many maximal elements?

These results in $\$ 7$ together provide a strategy (presented in detail in Theorem 7.15) for attempting to show that tight closure of a given proper ideal $\mathfrak{a}$ of $R$ commutes with localization and attempting to answer Katzman's question for a. A variant of the strategy that applies when the Frobenius powers of $\mathfrak{a}$ have linear growth of primary decompositions and $\bigcup_{n \in \mathbb{N}_{0}}$ ass $\mathfrak{a}^{\left[p^{n}\right]}$ is finite is presented in Theorem 7.14, although this should be accompanied by the warning that Katzman [10] has provided an example of a proper ideal $\mathfrak{d}$ in a ring of the type under consideration in this paper for which $\bigcup_{n \in \mathbb{N}_{0}}$ ass $\mathfrak{d}^{\left[p^{n}\right]}$ is infinite!

Several applications of these strategies are presented in the final 8 . We point out now that in most of those applications, it was already known that tight closure commutes with localization, often from the paper of Aberbach-Hochster-Huneke [1] however, the numerous results from use of the strategies that show that, in various circumstances, the union of the associated primes of the tight closures of the Frobenius powers of a fixed ideal is a finite set are, we believe, new, and, in view of the above-mentioned question of Katzman, of some interest.

For some of the applications, primary decompositions of appropriate ideals of perfect closures are constructed by 'approximating', in some sense, the original 
ring $R$ by a regular commutative Noetherian ring of characteristic $p$. Here, we just mention two of the applications, to give the general flavour. One of the main results of 88 is that if $R$ is equidimensional and integral over a regular excellent subring $A$, and $\mathfrak{a}$ is the extension to $R$ of a proper ideal of $A$, then, provided $R$ has a weak test element, the set $\bigcup_{n \in \mathbb{N}_{0}}$ ass $\left(\mathfrak{a}^{\left[p^{n}\right]}\right)^{*}$ is finite. A corollary is that if $x_{1}, \ldots, x_{d}$ form a system of parameters for an equidimensional excellent local ring $R$, and $\mathfrak{a}$ is a proper ideal of $R$ generated by 'polynomials' in $x_{1}, \ldots, x_{d}$ with coefficients in the prime subfield of $R$, then the set $\bigcup_{n \in \mathbb{N}_{0}}$ ass $\left(\mathfrak{a}^{\left[p^{n}\right]}\right)^{*}$ is finite.

\section{The PERFECT Closure}

For a commutative ring $R$ of prime characteristic $p$ we shall always denote by $f: R \longrightarrow R$ the Frobenius homomorphism, for which $f(r)=r^{p}$ for all $r \in R$. Recall that $R$ is said to be perfect if $f$ is an isomorphism.

1.1. Definition. M. J. Greenberg [2, $\S 2]$ proved that, for a general, not necessarily perfect, $R$ of the above type, there exists a pair $\left(R^{\infty}, \phi\right)$, where $R^{\infty}$ is a perfect ring and $\phi: R \longrightarrow R^{\infty}$ is a ring homomorphism such that, for any other such pair $\left(R^{\prime}, \phi^{\prime}\right)$, there exists a unique ring homomorphism $\psi: R^{\infty} \longrightarrow R^{\prime}$ with $\psi \circ \phi=\phi^{\prime}$; furthermore, $\operatorname{Ker} \phi=\sqrt{(0)}$, the nilradical of $R$, and if $\alpha \in R^{\infty}$, then $\alpha^{p^{m}} \in \phi(R)$ for some $m \geq 0$. The ring $R^{\infty}$ is referred to as the perfect closure of $R$.

1.2. Remark. It follows from Greenberg's work cited in 1.1 that, with the notation of that definition, $R^{\infty}$ can be constructed by forming the perfect closure of the reduced ring $R_{\text {red }}:=R / \sqrt{(0)}$. In $\$ 3$ below, we shall provide a concrete method of construction of the perfect closure of a reduced commutative ring of prime characteristic.

1.3. Remark. It also follows from Greenberg's work cited in 1.1 that if $R \cong \prod_{i=1}^{n} R_{i}$ is a finite product of commutative rings of the same prime characteristic, then $R^{\infty} \cong \prod_{i=1}^{n} R_{i}^{\infty}$.

1.4. Remark. Let $A$ be a subring of a commutative ring $A^{\prime}$ of prime characteristic $p$. Then $A^{\prime}$ is said to be a purely inseparable extension of $A$ if, for every element $a^{\prime} \in A^{\prime}$, there exists $n=n\left(a^{\prime}\right) \in \mathbb{N}_{0}$ such that $a^{\prime p^{n}} \in A$. It is easy to deduce from 1.1 that this is the case if and only if the ring homomorphism $\iota^{\infty}: A^{\infty} \longrightarrow A^{\prime \infty}$ induced by the inclusion homomorphism $\iota: A \stackrel{\subseteq}{\longrightarrow} A^{\prime}$ is an isomorphism.

\section{JORDAN'S CONSTRUCTION}

Throughout this section, let $A$ denote a (not necessarily commutative) ring (with identity), and let $g: A \longrightarrow A$ be an injective ring homomorphism. In [9], D. A. Jordan constructed in this situation a ring $A^{\prime}$ containing $A$ as a subring such that $g$ extends to $A^{\prime}$ and is an automorphism of $A^{\prime}$; the same paper provides a detailed description of the left ideals of $A^{\prime}$. We now recall his construction of $A^{\prime}$.

2.1. Definition. The skew polynomial ring $A[x, g]$ associated to $A$ and $g$ in an indeterminate $x$ over $A$ is, as a left $A$-module, freely generated by $\left(x^{i}\right)_{i \in \mathbb{N}_{0}}$, and so consists of all polynomials $\sum_{i=0}^{n} a_{i} x^{i}$, where $n \in \mathbb{N}_{0}$ and $a_{0}, \ldots, a_{n} \in A$; however, its multiplication is subject to the rule

$$
x a=g(a) x \quad \text { for all } a \in A .
$$


The (generalized) skew Laurent polynomial ring $A\left[x, x^{-1}, g\right]$ associated to $A$ and $g$ is the left quotient ring of $A[x, g]$ with respect to the left denominator set $\left\{x^{i}: i \in \mathbb{N}_{0}\right\}$. Hence elements in $A\left[x, x^{-1}, g\right]$ are finite sums of elements of the form $x^{-j} a x^{i}$, where $a \in A$ and $i, j \in \mathbb{N}_{0}$ and multiplication in $A\left[x, x^{-1}, g\right]$ is subject to the rules

$$
x a=g(a) x, \quad a x^{-1}=x^{-1} g(a) \quad \text { for all } a \in A .
$$

(In the case when $g$ is an automorphism, the elements of $A\left[x, x^{-1}, g\right]$ can be written in the form $\sum_{i=m}^{n} a_{i} x^{i}$, where $m, n \in \mathbb{Z}$ and $a_{m}, \ldots, a_{n} \in A$. Then $A\left[x, x^{-1}, g\right]$ is the standard skew Laurent polynomial ring.)

Let $A^{\prime}$ be the subring of the skew Laurent polynomial ring $A\left[x, x^{-1}, g\right]$ consisting of all elements of the form $x^{-i} a x^{i}$ with $i \in \mathbb{N}_{0}$ and $a \in A$. As $x^{-i} a x^{i}=$ $x^{-(i+j)} g^{j}(a) x^{i+j}$ for all $j \in \mathbb{N}_{0}$, this is a subring of $A\left[x, x^{-1}, g\right]$ with

$$
\begin{aligned}
x^{-i} a x^{i}+x^{-j} b x^{j} & =x^{-(i+j)}\left(g^{j}(a)+g^{i}(b)\right) x^{i+j}, \\
\left(x^{-i} a x^{i}\right)\left(x^{-j} b x^{j}\right) & =x^{-(i+j)}\left(g^{j}(a) g^{i}(b)\right) x^{i+j}
\end{aligned}
$$

for all $i, j \in \mathbb{N}_{0}$ and $a, b \in A$. The ring homomorphism $g$ extends to $A^{\prime}$ via $g\left(x^{-i} a x^{i}\right)=x^{-i} g(a) x^{i}$ for all $i \in \mathbb{N}_{0}$ and $a \in A$; in fact, $g: A^{\prime} \longrightarrow A^{\prime}$ is a ring isomorphism.

Jordan's philosophy in [9] is based on the fact that the skew Laurent polynomial rings $A\left[x, x^{-1}, g\right]$ and $A^{\prime}\left[x, x^{-1}, g\right]$ are isomorphic; this means that one may tackle problems concerning the structure of $A\left[x, x^{-1}, g\right]$ by reducing to the more familiar case where $g$ is an automorphism, provided that one can handle the relationship between $A$ and $A^{\prime}$. To this end, Jordan provided in $[9, \S 4]$ a comprehensive description of the left ideals of $A^{\prime}$. We review this next.

2.2. Definitions. Let the situation be as in 2.1

(i) Jordan calls a left ideal $I$ of $A$ closed if $g^{-n}\left(A g^{n}(I)\right)\left(:=\left(g^{n}\right)^{-1}\left(A g^{n}(I)\right)\right)$ $\subseteq I$ for all $n \in \mathbb{N}$.

The argument in the proof of [9. Proposition 4.4(i)] shows that if $J$ is an arbitrary left ideal of $A$ and $k \in \mathbb{N}_{0}$, then $\rho_{k}(J):=\bigcup_{n \in \mathbb{N}_{0}} g^{-n}\left(A g^{n+k}(J)\right)$ is always a closed left ideal of $A$.

(ii) Jordan says that a sequence $\left(I_{n}\right)_{n \in \mathbb{N}_{0}}$ of left ideals of $A$ is a $g$-sequence if $g^{-1}\left(I_{n+1}\right)=I_{n}$ for all $n \in \mathbb{N}_{0}$. (Actually, Jordan also required that all of the left ideals $I_{n}$ in the sequence be closed; however, this is an automatic consequence of the property that $g^{-1}\left(I_{n+1}\right)=I_{n}$ for all $n \in \mathbb{N}_{0}$, as we now show. Let $n \in \mathbb{N}_{0}$ and $i \in \mathbb{N}$. Since $g^{-i}\left(I_{n+i}\right)=I_{n}$, we have $A g^{i}\left(I_{n}\right) \subseteq I_{n+i}$. Therefore $g^{-i}\left(A g^{i}\left(I_{n}\right)\right) \subseteq g^{-i}\left(I_{n+i}\right)=I_{n}$. This shows that $I_{n}$ is closed.)

(iii) If $J$ is a left ideal of $A$, then the argument in the proof of [9. Proposition 4.4(ii)] shows that $\left(\rho_{n}(J)\right)_{n \in \mathbb{N}_{0}}$ is a $g$-sequence: we refer to this as the canonical g-sequence associated to $J$.

(iv) Jordan defined [9, p. 439] a natural partial order on the set of $g$-sequences of left ideals of $A$ : for such $g$-sequences $\left(I_{n}\right)_{n \in \mathbb{N}_{0}}$ and $\left(J_{n}\right)_{n \in \mathbb{N}_{0}}$, we write

$$
\left(I_{n}\right)_{n \in \mathbb{N}_{0}} \leq\left(J_{n}\right)_{n \in \mathbb{N}_{0}} \text { if and only if } I_{n} \subseteq J_{n} \text { for all } n \in \mathbb{N}_{0} .
$$

(v) We shall occasionally find it convenient to use the ring homomorphisms $\phi_{n}: A \longrightarrow A^{\prime}\left(n \in \mathbb{N}_{0}\right)$ defined by $\phi_{n}(a)=x^{-n} a x^{n}$ for all $a \in A$ (and $\left.n \in \mathbb{N}_{0}\right)$. 
2.3. Theorem (Jordan). Let the situation be as in 2.1. There is an order-preserving bijection, $\Gamma$, from the set of left ideals of $A^{\prime}$, partially ordered by inclusion, to the partially-ordered set of $g$-sequences of left ideals of $A$ given by

$\Gamma: I \longmapsto\left(I_{n}\right)_{n \in \mathbb{N}_{0}} \quad$ where $I_{n}:=\left\{a \in A: x^{-n} a x^{n} \in I\right\}=\phi_{n}^{-1}(I)$ for all $n \in \mathbb{N}_{0}$.

The inverse bijection, $\Gamma^{-1}$, also order-preserving, is given by

$$
\Gamma^{-1}:\left(I_{n}\right)_{n \in \mathbb{N}_{0}} \longmapsto \bigcup_{n \in \mathbb{N}_{0}} x^{-n} I_{n} x^{n}=\bigcup_{n \in \mathbb{N}_{0}} \phi_{n}\left(I_{n}\right) .
$$

If, with the notation of the above theorem, $\Gamma(I)=\left(I_{n}\right)_{n \in \mathbb{N}_{0}}$, then we shall say that $\left(I_{n}\right)_{n \in \mathbb{N}_{0}}$ is the $g$-sequence associated to, or corresponding to, $I$, and, given a $g$ sequence $\left(I_{n}\right)_{n \in \mathbb{N}_{0}}$, we shall call the left ideal $\Gamma^{-1}\left(\left(I_{n}\right)_{n \in \mathbb{N}_{0}}\right)$ the left ideal associated to, or corresponding to, the $g$-sequence $\left(I_{n}\right)_{n \in \mathbb{N}_{0}}$.

\section{Application of Jordan's CONSTRUCtion to PerfeCt ClOSURES}

In this section, we explain the relevance of Jordan's construction of $\$ 2$ to the perfect closure of a reduced commutative Noetherian ring $R$ of prime characteristic $p$ (and $R$ will have this meaning throughout this section). Since $R$ is reduced, the Frobenius homomorphism $f: R \longrightarrow R$ is injective, and we may take $f: R \longrightarrow R$ for the $g: A \longrightarrow A$ in Jordan's construction of $\S 2$

3.1. Remark. We apply Jordan's construction with $f: R \longrightarrow R$ in the rôle of $g: A \longrightarrow A$.

(i) The ring $A^{\prime}$ (which is commutative in this case) is just the perfect closure of $R$, because if $R^{\prime}$ is a perfect commutative ring and $\phi^{\prime}: R \longrightarrow R^{\prime}$ is a ring homomorphism, then there is a unique ring homomorphism $\psi: A^{\prime} \longrightarrow R^{\prime}$ whose restriction to $R$ is $\phi^{\prime}$, given by $\psi\left(x^{-n} r x^{n}\right)=\phi^{\prime}(r)^{1 / p^{n}}$, the unique $p^{n}$-th root of $\phi^{\prime}(r)$ in $R^{\prime}$, for all $n \in \mathbb{N}_{0}$ and $r \in R$.

We shall denote $A^{\prime}$ in this case by $R^{\infty}$. Observe that, for $n \in \mathbb{N}_{0}$ and $r \in R$, the element $x^{-n} r x^{n}$ of $R^{\infty}$ is the unique $p^{n}$-th root $r^{1 / p^{n}}$ of $r$ in $R^{\infty}$, and the ring homomorphism $\phi_{n}: R \longrightarrow R^{\infty}$ of 2.2(v) maps $r$ to $r^{1 / p^{n}}$. Thus Jordan's construction provides a rather concrete presentation of the perfect closure of $R$.

(ii) A sequence $\left(\mathfrak{a}_{n}\right)_{n \in \mathbb{N}_{0}}$ of ideals of $R$ is an $f$-sequence if $f^{-1}\left(\mathfrak{a}_{n+1}\right)=\mathfrak{a}_{n}$ for all $n \in \mathbb{N}_{0}$.

Of course, Jordan's Theorem 2.3 provides detailed information about the ideals of $R^{\infty}$.

3.2. Corollary. There is an order-preserving bijection, $\Gamma$, from the set of ideals of $R^{\infty}$, partially ordered by inclusion, to the partially-ordered set of $f$-sequences of ideals of $R$ given by

$$
\Gamma: \mathfrak{A} \longmapsto\left(\mathfrak{a}_{n}\right)_{n \in \mathbb{N}_{0}}
$$

where

$$
\mathfrak{a}_{n}:=\left\{r \in R: x^{-n} r x^{n}=r^{1 / p^{n}} \in \mathfrak{A}\right\}=\phi_{n}^{-1}(\mathfrak{A}) \text { for all } n \in \mathbb{N}_{0} .
$$

The inverse bijection, $\Gamma^{-1}$, also order-preserving, is given by

$$
\Gamma^{-1}:\left(\mathfrak{a}_{n}\right)_{n \in \mathbb{N}_{0}} \longmapsto \bigcup_{n \in \mathbb{N}_{0}} x^{-n} \mathfrak{a}_{n} x^{n}=\bigcup_{n \in \mathbb{N}_{0}} \phi_{n}\left(\mathfrak{a}_{n}\right) .
$$




\section{Properties of $f$-Sequences}

Throughout this section and the remainder of the paper, $R$ will denote a commutative Noetherian ring of prime characteristic $p$; note that we have dropped the hypothesis that $R$ be reduced. In this section, we extend the concept of $f$-sequence of ideals to this more general situation, and then develop the concept in some detail.

4.1. Definitions. Let $\mathfrak{a}$ and $\mathfrak{b}$ be ideals of $R$.

(i) The ideal $\mathfrak{a}$ of $R$ is said to be $F$-closed if, whenever $r \in R$ is such that $r^{p^{n}} \in \mathfrak{a}^{\left[p^{n}\right]}$ for some $n \in \mathbb{N}_{0}$ (or, equivalently, for all $n \gg 0$ ), then $r \in \mathfrak{a}$.

(ii) An $f$-sequence of ideals of $R$ is a sequence $\left(\mathfrak{a}_{n}\right)_{n \in \mathbb{N}_{0}}$ of ideals of $R$ such that $f^{-1}\left(\mathfrak{a}_{n+1}\right)=\mathfrak{a}_{n}$ for all $n \in \mathbb{N}_{0}$. There is a partial order on the set of such $f$-sequences, defined analogously to the partial order of 2.2(iv). The argument of 2.2(ii) applies here to show that every term in an $f$-sequence is $F$-closed.

(iii) The $F$-closure of $\mathfrak{b}$ is the $\left(F\right.$-closed) ideal $\mathfrak{b}^{F}:=\bigcup_{n \in \mathbb{N}_{0}} f^{-n}\left(R f^{n}(\mathfrak{b})\right)=$ $\bigcup_{n \in \mathbb{N}_{0}} f^{-n}\left(\mathfrak{b}^{\left[p^{n}\right]}\right)$, and $\left(\left(\mathfrak{b}^{\left[p^{n}\right]}\right)^{F}\right)_{n \in \mathbb{N}_{0}}$ is an $f$-sequence, called the canonical $f$-sequence associated to $\mathfrak{b}$.

4.2. Remark. Let $\left(\mathfrak{a}_{n}\right)_{n \in \mathbb{N}_{0}}$ be an $f$-sequence of ideals of $R$.

(i) The $f$-sequence $\left(\mathfrak{a}_{n}\right)_{n \in \mathbb{N}_{0}}$ is descending, because if $a \in \mathfrak{a}_{n+1}$ for some $n \in \mathbb{N}_{0}$, then $f(a)=a^{p} \in \mathfrak{a}_{n+1}$, so that $a \in f^{-1}\left(\mathfrak{a}_{n+1}\right)=\mathfrak{a}_{n}$. Further, $\mathfrak{a}_{n}^{[p]} \subseteq \mathfrak{a}_{n+1}$ since $f\left(\mathfrak{a}_{n}\right) \subseteq \mathfrak{a}_{n+1}$.

This implies, as $\mathfrak{a}_{n+1}$ is $F$-closed, that $\mathfrak{a}_{n}^{[p]} \subseteq\left(\mathfrak{a}_{n}^{[p]}\right)^{F} \subseteq \mathfrak{a}_{n+1} \subseteq \mathfrak{a}_{n}$, so that $\sqrt{\mathfrak{a}_{n}}=\sqrt{\mathfrak{a}_{n+1}}$, for all $n \in \mathbb{N}_{0}$.

Similar arguments show that $\left(\mathfrak{a}_{n}^{\left[p^{j}\right]}\right)^{F} \subseteq \mathfrak{a}_{n+j}$ for all $n, j \in \mathbb{N}_{0}$.

(ii) Each term $\mathfrak{a}_{n}$ in the $f$-sequence contains $\sqrt{(0)}$, since $\mathfrak{a}_{n}$ is $F$-closed.

(iii) We shall sometimes need to use the fact that if, for some $t \in \mathbb{N}$, one has a sequence $\left(\mathfrak{b}_{n}\right)_{n \geq t}$ of ideals of $R$ such that $f^{-1}\left(\mathfrak{b}_{n+1}\right)=\mathfrak{b}_{n}$ for all $n \geq t$, then there is exactly one way of extending the sequence 'downwards' to an $f$-sequence $\left(\mathfrak{b}_{n}\right)_{n \in \mathbb{N}_{0}}$. One achieves this extension by setting $\mathfrak{b}_{n}=$ $f^{-(t-n)}\left(\mathfrak{b}_{t}\right)$ for all $n=t-1, \ldots, 0$.

(iv) Note that ass $\mathfrak{a}_{n} \subseteq$ ass $\mathfrak{a}_{n+1}$ for each $n \in \mathbb{N}_{0}$, since $f^{-1}\left(\mathfrak{a}_{n+1}\right)=\mathfrak{a}_{n}$.

(v) Note also that all the terms of the $f$-sequence $\left(\mathfrak{a}_{n}\right)_{n \in \mathbb{N}_{0}}$ have the same set of minimal primes, since $\sqrt{\mathfrak{a}_{n}}=\sqrt{\mathfrak{a}_{n+1}}$ for all $n \in \mathbb{N}_{0}$, by (i). The members of the common set of minimal primes of the terms of the $f$-sequence are referred to as the minimal primes of the $f$-sequence; note that there are only finitely many of these minimal primes.

The fact that every term in an $f$-sequence of ideals of $R$ contains the nilradical of $R$ (by 4.2 (ii)) means that there is an obvious bijective correspondence (given by extension of the terms and contraction of the terms) between the set of $f$-sequences of ideals of $R$ and the set of $f$-sequences of ideals of $R_{\text {red }}$. This means that Jordan's Corollary 3.2 has an analogue that applies in this more general situation.

4.3. Corollary. We shall use overlines to denote natural images in $R_{\text {red }}$ of elements of $R$ under the natural homomorphism $\pi: R \longrightarrow R_{\text {red }}$, although we shall sometimes omit the overlines when $R$ is itself reduced. We shall interpret the subring $A^{\prime}$ of the skew Laurent polynomial ring $R_{\mathrm{red}}\left[x, x^{-1}, f\right]$, as defined in [2.1, as the perfect closure $\left(R_{\text {red }}\right)^{\infty}=R^{\infty}$ of $R$. For each $n \in \mathbb{N}_{0}$, we shall use $\phi_{n}: R_{\text {red }} \longrightarrow R^{\infty}$ to 
denote the ring homomorphism for which $\phi_{n}(\bar{r})=x^{-n} \bar{r} x^{n}=\bar{r}^{1 / p^{n}}$ for all $r \in R$, and $\psi_{n}: R \longrightarrow R^{\infty}$ to denote the composition $\phi_{n} \circ \pi$.

There is an order-preserving bijection, $\Gamma$, from the set of ideals of $R^{\infty}$, partially ordered by inclusion, to the partially-ordered set of $f$-sequences of ideals of $R$ given by

$$
\Gamma: \mathfrak{A} \longmapsto\left(\mathfrak{a}_{n}\right)_{n \in \mathbb{N}_{0}}
$$

where

$$
\mathfrak{a}_{n}:=\left\{r \in R: x^{-n} \bar{r} x^{n}=\bar{r}^{1 / p^{n}} \in \mathfrak{A}\right\}=\psi_{n}^{-1}(\mathfrak{A}) \text { for all } n \in \mathbb{N}_{0} .
$$

The inverse bijection, $\Gamma^{-1}$, also order-preserving, is given by

$$
\Gamma^{-1}:\left(\mathfrak{a}_{n}\right)_{n \in \mathbb{N}_{0}} \longmapsto \bigcup_{n \in \mathbb{N}_{0}} x^{-n} \pi\left(\mathfrak{a}_{n}\right) x^{n}=\bigcup_{n \in \mathbb{N}_{0}} \psi_{n}\left(\mathfrak{a}_{n}\right) .
$$

4.4. Lemma. Let $\eta: R \longrightarrow R^{\prime}$ be a homomorphism of commutative Noetherian rings of characteristic $p$, and let $\left(\mathfrak{a}_{n}^{\prime}\right)_{n \in \mathbb{N}_{0}}$ be an $f$-sequence of ideals of $R^{\prime}$ corresponding to the ideal $\mathfrak{A}^{\prime}$ of $R^{\prime \infty}$. Let $\eta^{\infty}: R^{\infty} \longrightarrow R^{\prime \infty}$ be the ring homomorphism induced by $\eta$ (see $§ 1$ ).

Then the $f$-sequence of ideals of $R$ corresponding to the ideal $\left(\eta^{\infty}\right)^{-1}\left(\mathfrak{A}^{\prime}\right)$ of $R^{\infty}$ is $\left(\eta^{-1}\left(\mathfrak{a}_{n}^{\prime}\right)\right)_{n \in \mathbb{N}_{0}}$.

Proof. Let $\psi_{n}: R \longrightarrow R^{\infty}$ be the ring homomorphism of 4.3 and let $\psi_{n}^{\prime}: R^{\prime} \longrightarrow$ $R^{\prime \infty}$ be the corresponding ring homomorphism for $R^{\prime}$. The claim follows easily from the fact that $\eta^{\infty} \circ \psi_{n}=\psi_{n}^{\prime} \circ \eta$ for all $n \in \mathbb{N}_{0}$.

\section{Examples of $f$-SEQUenCes}

Throughout this section, $R$ will denote a commutative Noetherian ring of prime characteristic $p$. The first two lemmas in this section present important examples of $f$-sequences.

5.1. Lemma. Let $\mathfrak{a}$ be an ideal of $R$. Then $\left(\left(\mathfrak{a}^{\left[p^{n}\right]}\right)^{*}\right)_{n \in \mathbb{N}_{0}}$, the sequence of tight closures of the Frobenius powers of $\mathfrak{a}$, is an $f$-sequence.

Proof. Let $n \in \mathbb{N}_{0}$.

Let $r \in f^{-1}\left(\left(\mathfrak{a}^{\left[p^{n+1}\right]}\right)^{*}\right)$, so that $r^{p} \in\left(\mathfrak{a}^{\left[p^{n+1}\right]}\right)^{*}$ and there exists $c \in R^{\circ}$ such that $c\left(r^{p}\right)^{p^{j}} \in\left(\mathfrak{a}^{\left[p^{n+1}\right]}\right)^{\left[p^{j}\right]}$ for all $j \gg 0$; hence $c r^{p^{j+1}} \in\left(\mathfrak{a}^{\left[p^{n}\right]}\right)^{\left[p^{j+1}\right]}$ for all $j \gg 0$, so that $r \in\left(\mathfrak{a}^{\left[p^{n}\right]}\right)^{*}$. Hence $f^{-1}\left(\left(\mathfrak{a}^{\left[p^{n+1}\right]}\right)^{*}\right) \subseteq\left(\mathfrak{a}^{\left[p^{n}\right]}\right)^{*}$.

Now let $r \in\left(\mathfrak{a}^{\left[p^{n}\right]}\right)^{*}$, so that there exists $c \in R^{\circ}$ such that $c r^{p^{j}} \in\left(\mathfrak{a}^{\left[p^{n}\right]}\right)^{\left[p^{j}\right]}$ for all $j \gg 0$. Then $c^{p} r^{p^{j+1}} \in\left(\left(\mathfrak{a}^{\left[p^{n}\right]}\right)^{\left[p^{j}\right]}\right)^{[p]}$ for all $j \gg 0$. Hence $c^{p}\left(r^{p}\right)^{p^{j}} \in\left(\mathfrak{a}^{\left[p^{n+1}\right]}\right)^{\left[p^{j}\right]}$ for all $j \gg 0$, and so $r^{p} \in\left(\mathfrak{a}^{\left[p^{n+1}\right]}\right)^{*}$ because $c^{p} \in R^{\circ}$.

The next lemma concerns the case where $R$ is a domain and involves the plus closure of an ideal $\mathfrak{a}$ of $R$ : the reader was reminded about this concept in the Introduction. In this situation, we denote by $R^{+}$the integral closure of $R$ in an algebraic closure of its field of fractions, and refer to $R^{+}$as the absolute integral closure of $R$. Recall from Huneke [7, p. 15] that $\mathfrak{a} \subseteq \mathfrak{a}^{+} \subseteq \mathfrak{a}^{*}$.

5.2. Lemma. Assume that $R$ is a domain and let $\mathfrak{a}$ be an ideal of $R$. Then $\left(\left(\mathfrak{a}^{\left[p^{n}\right]}\right)^{+}\right)_{n \in \mathbb{N}_{0}}$, the sequence of plus closures of the Frobenius powers of $\mathfrak{a}$, is an $f$-sequence. In fact, it is the $f$-sequence corresponding to the ideal $\mathfrak{a} R^{+} \cap R^{\infty}$ of $R^{\infty}$ in the correspondence of 3.2 . 
Proof. It is enough to prove the second statement. Let $\left(\mathfrak{a}_{n}\right)_{n \in \mathbb{N}_{0}}$ be the $f$-sequence of ideals of $R$ that corresponds to the ideal $\mathfrak{a} R^{+} \cap R^{\infty}$ of $R^{\infty}$. Let $n \in \mathbb{N}_{0}$, and suppose that $a_{1}, \ldots, a_{t}$ generate $\mathfrak{a}$. Note that $\mathfrak{a}_{n}=\left\{r \in R: r^{1 / p^{n}} \in \mathfrak{a} R^{+} \cap R^{\infty}\right\}$.

Let $r \in\left(\mathfrak{a}^{\left[p^{n}\right]}\right)^{+}$. Thus $r \in R$ and there exist $\sigma_{1}, \ldots, \sigma_{t} \in R^{+}$such that $r=$ $a_{1}^{p^{n}} \sigma_{1}+\cdots+a_{t}^{p^{n}} \sigma_{t}$. Now, for each $i=1, \ldots, t$, the unique $p^{n}$-th $\operatorname{root} \sigma_{i}^{1 / p^{n}}$ of $\sigma_{i}$ in the algebraic closure of the field of fractions of $R$ is integral over $R^{+}$, and so belongs to $R^{+}$. Hence $r^{1 / p^{n}}=a_{1} \sigma_{1}^{1 / p^{n}}+\cdots+a_{t} \sigma_{t}^{1 / p^{n}} \in \mathfrak{a} R^{+} \cap R^{\infty}$. Therefore $r \in \mathfrak{a}_{n}$. Thus $\left(\mathfrak{a}^{\left[p^{n}\right]}\right)^{+} \subseteq \mathfrak{a}_{n}$. The reverse inclusion is even easier.

We now drop the hypothesis that $R$ is a domain, and revert to our standard hypotheses about $R$. We would like to have a variant of the concept of plus closure available for use in situations where $R$ is not a domain (and not even reduced), and the following definition introduces a suitable one.

5.3. Definition and remarks. Suppose that there are $h$ minimal prime ideals $\mathfrak{p}_{1}, \ldots, \mathfrak{p}_{h}$ of $R$; let $\mathfrak{a}$ be an ideal of $R$. For each $i=1, \ldots, h$, one can use the integral domain $R / \mathfrak{p}_{i}$ to construct $\left(\left(R / \mathfrak{p}_{i}\right)^{+}\right.$and $)$the plus closure $\left(\left(\mathfrak{a}+\mathfrak{p}_{i}\right) / \mathfrak{p}_{i}\right)^{+}$of the ideal $\left(\mathfrak{a}+\mathfrak{p}_{i}\right) / \mathfrak{p}_{i}$ of $R / \mathfrak{p}_{i}$.

We define the plus closure $\mathfrak{a}^{+}$of $\mathfrak{a}$ to be the contraction back to $R$ of the ideal $\prod_{i=1}^{h}\left(\left(\mathfrak{a}+\mathfrak{p}_{i}\right) / \mathfrak{p}_{i}\right)^{+}$of the direct product $\prod_{i=1}^{h} R / \mathfrak{p}_{i}$ under the natural ring homomorphism $\nu: R \longrightarrow \prod_{i=1}^{h} R / \mathfrak{p}_{i}$.

Note that, in view of $\S 1$, one can identify $\left(\prod_{i=1}^{h} R / \mathfrak{p}_{i}\right)^{\infty}$ with $\prod_{i=1}^{h}\left(R / \mathfrak{p}_{i}\right)^{\infty}$ under an isomorphism that maps

$$
\left(\left(r_{1}+\mathfrak{p}_{1}\right), \ldots,\left(r_{h}+\mathfrak{p}_{h}\right)\right)^{1 / p^{n}}
$$

to

$$
\left(\left(r_{1}+\mathfrak{p}_{1}\right)^{1 / p^{n}}, \ldots,\left(r_{h}+\mathfrak{p}_{h}\right)^{1 / p^{n}}\right)
$$

for $r_{1}, \ldots, r_{h} \in R$ and $n \in \mathbb{N}_{0}$, and there is an induced injective ring homomorphism

$$
\nu^{\infty}: R^{\infty}=\left(R_{\text {red }}\right)^{\infty} \longrightarrow \prod_{i=1}^{h}\left(R / \mathfrak{p}_{i}\right)^{\infty}=\left(\prod_{i=1}^{h} R / \mathfrak{p}_{i}\right)^{\infty}
$$

such that

$$
\nu^{\infty}\left(\bar{r}^{1 / p^{n}}\right)=\left(\left(r+\mathfrak{p}_{1}\right)^{1 / p^{n}}, \ldots,\left(r+\mathfrak{p}_{h}\right)^{1 / p^{n}}\right) \text { for all } r \in R \text { and } n \in \mathbb{N}_{0} .
$$

We use $\nu^{\infty}$ to identify $R^{\infty}$ as a subring of $\prod_{i=1}^{h}\left(R / \mathfrak{p}_{i}\right)^{\infty}$; the latter ring is identified with a subring of $\prod_{i=1}^{h}\left(R / \mathfrak{p}_{i}\right)^{+}$in the obvious natural way. Note that the diagram

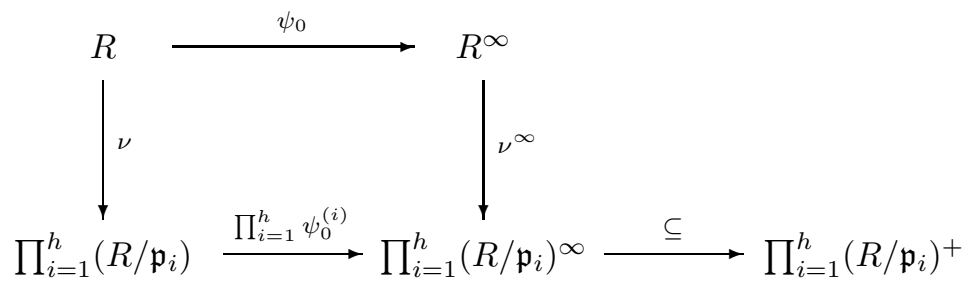


(in which $\psi_{0}: R \longrightarrow R^{\infty}$ and $\psi_{0}^{(i)}: R / \mathfrak{p}_{i} \longrightarrow\left(R / \mathfrak{p}_{i}\right)^{\infty}(i=1, \ldots, h)$ are the natural ring homomorphisms) commutes, and

$$
\begin{aligned}
\mathfrak{a}^{+} & =\nu^{-1}\left(\prod_{i=1}^{h}\left(\left(\mathfrak{a}+\mathfrak{p}_{i}\right) / \mathfrak{p}_{i}\right)^{+}\right) \\
& =\nu^{-1}\left(\left(\prod_{i=1}^{h} \psi_{0}^{(i)}\right)^{-1}\left(\prod_{i=1}^{h}\left(\left(\mathfrak{a}+\mathfrak{p}_{i}\right) / \mathfrak{p}_{i}\right)\left(R / \mathfrak{p}_{i}\right)^{+} \cap\left(R / \mathfrak{p}_{i}\right)^{\infty}\right)\right) \\
& =\left(\psi_{0}\right)^{-1}\left(\left(\nu^{\infty}\right)^{-1}\left(\left(\prod_{i=1}^{h}\left(\left(\mathfrak{a}+\mathfrak{p}_{i}\right) / \mathfrak{p}_{i}\right)\left(R / \mathfrak{p}_{i}\right)^{+}\right) \cap\left(\prod_{i=1}^{h}\left(R / \mathfrak{p}_{i}\right)^{\infty}\right)\right)\right) \\
& =\left(\psi_{0}\right)^{-1}\left(\left(\prod_{i=1}^{h}\left(\left(\mathfrak{a}+\mathfrak{p}_{i}\right) / \mathfrak{p}_{i}\right)\left(R / \mathfrak{p}_{i}\right)^{+}\right) \cap R^{\infty}\right) .
\end{aligned}
$$

When $R$ is a domain, $\mathfrak{a}^{+}$, as defined in [5.3, coincides with the plus closure of $\mathfrak{a}$, as defined by Hochster and Huneke. The next lemma demonstrates that, in the general case, plus closure retains one important property of the Hochster-Huneke concept.

5.4. Lemma. Let $\mathfrak{a}$ be an ideal of $R$, and use the notation of 5.3 ,

(i) We have $\mathfrak{a} \subseteq \mathfrak{a}^{+} \subseteq \mathfrak{a}^{*}$.

(ii) The $f$-sequence of ideals of $\prod_{i=1}^{h} R / \mathfrak{p}_{i}$ to which the ideal

$$
\begin{array}{r}
\prod_{i=1}^{h}\left(\left(\mathfrak{a}+\mathfrak{p}_{i}\right) / \mathfrak{p}_{i}\right)\left(R / \mathfrak{p}_{i}\right)^{+} \cap\left(R / \mathfrak{p}_{i}\right)^{\infty} \\
\text { of } \prod_{i=1}^{h}\left(R / \mathfrak{p}_{i}\right)^{\infty}=\left(\prod_{i=1}^{h} R / \mathfrak{p}_{i}\right)^{\infty} \text { corresponds is } \\
\left(\prod_{i=1}^{h}\left(\left(\mathfrak{a}^{\left[p^{n}\right]}+\mathfrak{p}_{i}\right) / \mathfrak{p}_{i}\right)^{+}\right)_{n \in \mathbb{N}_{0}} .
\end{array}
$$

(iii) Also, $\left(\left(\mathfrak{a}^{\left[p^{n}\right]}\right)^{+}\right)_{n \in \mathbb{N}_{0}}$ is the $f$-sequence of ideals of $R$ corresponding to the ideal

$$
\left(\prod_{i=1}^{h}\left(\left(\mathfrak{a}+\mathfrak{p}_{i}\right) / \mathfrak{p}_{i}\right)\left(R / \mathfrak{p}_{i}\right)^{+}\right) \cap R^{\infty}
$$

of $R^{\infty}$.

Proof. (i) It is clear that $\mathfrak{a} \subseteq \mathfrak{a}^{+}$. Let $r \in \mathfrak{a}^{+}$. Then

$$
\nu(r)=\left(r+\mathfrak{p}_{1}, \ldots, r+\mathfrak{p}_{h}\right) \in \prod_{i=1}^{h}\left(\left(\mathfrak{a}+\mathfrak{p}_{i}\right) / \mathfrak{p}_{i}\right)^{+} \subseteq \prod_{i=1}^{h}\left(\left(\mathfrak{a}+\mathfrak{p}_{i}\right) / \mathfrak{p}_{i}\right)^{*} \subseteq \prod_{i=1}^{h} R / \mathfrak{p}_{i} .
$$

It then follows from [7, Theorem 1.3(c)] that $r \in \mathfrak{a}^{*}$.

(ii) This is an easy consequence of 5.2 .

(iii) This now follows easily from Lemma 4.4 applied to the ring homomorphism $\nu: R \longrightarrow \prod_{i=1}^{h} R / \mathfrak{p}_{i}$.

The next lemma will help us to construct new examples of $f$-sequences from known ones. 
5.5. Lemma. Let $\left(\mathfrak{a}_{n}\right)_{n \in \mathbb{N}_{0}}$ be an $f$-sequence of ideals of $R$ and let $\mathfrak{A}$ be the corresponding ideal of $R^{\infty}$. Let $S$ be a multiplicatively closed subset of $R$, and, with the notation of 4.3 , set $T:=\bigcup_{n \in \mathbb{N}_{0}} x^{-n} \pi(S) x^{n}$, a multiplicatively closed subset of $R^{\infty}$. Further let ${ }^{e}$ and ${ }^{c}$ stand for extension and contraction with respect to the natural ring homomorphism $R \longrightarrow S^{-1} R$, and let ${ }^{E}$ and ${ }^{C}$ stand for extension and contraction with respect to the natural ring homomorphism $R^{\infty} \longrightarrow T^{-1}\left(R^{\infty}\right)$.

Then $\left(\mathfrak{a}_{n}^{e c}\right)_{n \in \mathbb{N}_{0}}$ is also an $f$-sequence of ideals of $R$, and the ideal of $R^{\infty}$ to which it corresponds is $\mathfrak{A}^{E C}$.

Proof. It is straightforward to check that $T$ is a multiplicatively closed subset of $R^{\infty}$. Thus $\mathfrak{A}^{E C}$ is an ideal of $R^{\infty}$. Let $\left(\mathfrak{a}_{n}^{\prime}\right)_{n \in \mathbb{N}_{0}}$ be the corresponding $f$-sequence of ideals of $R$. It is enough for us to show that $\mathfrak{a}_{n}^{\prime}=\mathfrak{a}_{n}^{e c}$ for each $n \in \mathbb{N}_{0}$. Note that $\mathfrak{a}_{n}^{\prime}=\left\{a \in R: x^{-n} \bar{a} x^{n} \in \mathfrak{A}^{E C}\right\}$.

Let $a \in \mathfrak{a}_{n}^{e c}$. Thus there exists $s \in S$ such that $s a \in \mathfrak{a}_{n}$. Hence $x^{-n} \overline{s a} x^{n} \in \mathfrak{A}$, so that $x^{-n} \bar{s} x^{n} x^{-n} \bar{a} x^{n} \in \mathfrak{A}$. Since $x^{-n} \bar{s} x^{n} \in T$, this means that $x^{-n} \bar{a} x^{n} \in \mathfrak{A}^{E C}$ and $a \in \mathfrak{a}_{n}^{\prime}$.

Now let $a \in \mathfrak{a}_{n}^{\prime}$, so that $x^{-n} \bar{a} x^{n} \in \mathfrak{A}^{E C}$ and there exists $\tau \in T$ such that $\tau x^{-n} \bar{a} x^{n} \in \mathfrak{A}$. We can write $\tau=x^{-k} \bar{s} x^{k}$ for some $k \in \mathbb{N}_{0}$ and $s \in S$. If $k \leq n$, then

$$
x^{-n} \overline{s^{p^{n-k}} a} x^{n}=x^{-n} \bar{s}^{p^{n-k}} x^{n} x^{-n} \bar{a} x^{n}=x^{-k} \bar{s} x^{k} x^{-n} \bar{a} x^{n}=\tau x^{-n} \bar{a} x^{n} \in \mathfrak{A} .
$$

Hence, in this case, $s^{p^{n-k}} a \in \mathfrak{a}_{n}$, so that $a \in \mathfrak{a}_{n}^{e c}$ because $s^{p^{n-k}} \in S$.

We now consider the case where $k>n$. Then

$$
x^{-n} \overline{s a} x^{n}=x^{-n} \bar{s} x^{n} x^{-n} \bar{a} x^{n}=x^{-k} \bar{s}^{p^{k-n}} x^{k} x^{-n} \bar{a} x^{n}=x^{-k} \bar{s}^{p^{k-n}-1} x^{k}\left(\tau x^{-n} \bar{a} x^{n}\right) \in \mathfrak{A} .
$$

Therefore $s a \in \mathfrak{a}_{n}$, so that $a \in \mathfrak{a}_{n}^{e c}$.

\section{Primary decompositions in Perfect Closures}

Throughout this section, $R$ will denote a commutative Noetherian ring of prime characteristic $p$. Our aim in the section is to establish necessary and sufficient conditions for a proper ideal $\mathfrak{A}$ of the perfect closure $R^{\infty}$ to have a primary decomposition; our conditions will be phrased in terms of the $f$-sequence of ideals of $R$ that corresponds to $\mathfrak{A}$.

6.1. Theorem. Let $\mathfrak{A}$ be an ideal of $R^{\infty}$, and let $\left(\mathfrak{a}_{n}\right)_{n \in \mathbb{N}_{0}}$ be the corresponding $f$-sequence of ideals of $R$. We use the notation of 4.3 .

(i) The ideal $\mathfrak{A}$ is prime if and only if there is a $\mathfrak{p} \in \operatorname{Spec}(R)$ such that $\mathfrak{p}=\mathfrak{a}_{n}$ for all $n \in \mathbb{N}_{0}$. Thus the $f$-sequence corresponding to a prime ideal $\mathfrak{P}$ of $R^{\infty}$ is the constant $f$-sequence $\left(\pi^{-1}\left(\mathfrak{P} \cap R_{\text {red }}\right)\right)_{n \in \mathbb{N}_{0}}$, and the correspondence of 4.3 yields an inclusion-preserving bijective correspondence between $\operatorname{Spec}\left(R^{\infty}\right)$ and $\operatorname{Spec}(R)$.

(ii) The sequence $\left(\sqrt{\mathfrak{a}_{n}}\right)_{n \in \mathbb{N}_{0}}$ is an $f$-sequence, and it corresponds to the ideal $\sqrt{\mathfrak{A}}$ of $R^{\infty}$. Thus $\sqrt{\bigcup_{n \in \mathbb{N}_{0}} x^{-n} \pi\left(\mathfrak{a}_{n}\right) x^{n}}=\bigcup_{n \in \mathbb{N}_{0}} x^{-n} \pi\left(\sqrt{\mathfrak{a}_{n}}\right) x^{n}$.

(iii) The ideal $\mathfrak{A}$ is radical if and only if there is a radical ideal $\mathfrak{b}$ of $R$ such that $\mathfrak{b}=\mathfrak{a}_{n}$ for all $n \in \mathbb{N}_{0}$.

(iv) Let $\mathfrak{P} \in \operatorname{Spec}\left(R^{\infty}\right)$, and let $\mathfrak{p}$ be the corresponding prime ideal of $R$ : see (i) above. Then $\mathfrak{A}$ is a $\mathfrak{P}$-primary ideal of $R^{\infty}$ if and only if $\mathfrak{a}_{n}$ is $\mathfrak{p}$-primary for all $n \in \mathbb{N}_{0}$. 
(v) Let $\left(\mathfrak{A}_{\lambda}\right)_{\lambda \in \Lambda}$ be a family of ideals of $R^{\infty}$, and, for each $\lambda \in \Lambda$, let $\left(\mathfrak{a}_{\lambda, n}\right)_{n \in \mathbb{N}_{0}}$ be the $f$-sequence corresponding to $\mathfrak{A}_{\lambda}$. Then $\left(\bigcap_{\lambda \in \Lambda} \mathfrak{a}_{\lambda, n}\right)_{n \in \mathbb{N}_{0}}$ is the $f$ sequence of ideals of $R$ corresponding to the ideal $\bigcap_{\lambda \in \Lambda} \mathfrak{A}_{\lambda}$ of $R^{\infty}$. Consequently,

$$
\bigcup_{n \in \mathbb{N}_{0}}\left(x^{-n} \pi\left(\bigcap_{\lambda \in \Lambda} \mathfrak{a}_{\lambda, n}\right) x^{n}\right)=\bigcap_{\lambda \in \Lambda}\left(\bigcup_{n \in \mathbb{N}_{0}} x^{-n} \pi\left(\mathfrak{a}_{\lambda, n}\right) x^{n}\right) .
$$

Proof. Since each term in an $f$-sequence of ideals of $R$, and each prime ideal of $R$, contains $\sqrt{(0)}$, it is enough for us to prove this theorem under the additional assumption that $R$ is reduced; with this assumption, $\psi_{n}=\phi_{n}$ for all $n \in \mathbb{N}_{0}$.

(i) We have $f^{-1}\left(\mathfrak{a}_{n+1}\right)=\mathfrak{a}_{n}=\phi_{n}^{-1}(\mathfrak{A})$ for all $n \in \mathbb{N}_{0}$, and it is immediate from this that if $\mathfrak{A}$ is prime, then $\left(\mathfrak{a}_{n}\right)_{n \in \mathbb{N}_{0}}$ is constant with all its terms equal to the same prime ideal.

Conversely, if there exists $\mathfrak{p} \in \operatorname{Spec}(R)$ such that $\mathfrak{a}_{n}=\mathfrak{p}$ for all $n \in \mathbb{N}_{0}$, then it is routine to check that $\mathfrak{A}=\bigcup_{n \in \mathbb{N}_{0}} x^{-n} \mathfrak{p} x^{n} \in \operatorname{Spec}\left(R^{\infty}\right)$ : note that 1 cannot be written as $x^{-n} r x^{n}$ for any $r \in \mathfrak{p}$, and that if $a, b \in R$ are such that $x^{-i} a x^{i} x^{-j} b x^{j} \in \mathfrak{A}$ for some integers $i, j \in \mathbb{N}_{0}$, then $x^{-i-j} f^{j}(a) f^{i}(b) x^{i+j} \in \mathfrak{A}$, so that $f^{j}(a) f^{i}(b) \in$ $\mathfrak{a}_{i+j}=\mathfrak{p}$, from which it follows that $a$ or $b$ lies in $\mathfrak{p}$.

(ii) Of course, $\sqrt{\mathfrak{A}}$ is an ideal of $R^{\infty}$; by 4.3 , the $f$-sequence to which it corresponds is

$$
\left(\phi_{n}^{-1}(\sqrt{\mathfrak{A}})\right)_{n \in \mathbb{N}_{0}}=\left(\sqrt{\phi_{n}^{-1}(\mathfrak{A})}\right)_{n \in \mathbb{N}_{0}}=\left(\sqrt{\mathfrak{a}_{n}}\right)_{n \in \mathbb{N}_{0}} .
$$

(iii) The ideal $\mathfrak{A}$ is radical if and only if $\mathfrak{A}=\sqrt{\mathfrak{A}}$; by 4.3 this is the case if and only if the $f$-sequences to which $\mathfrak{A}$ and $\sqrt{\mathfrak{A}}$ correspond are the same; and it follows from part (ii) that this is the case if and only if $\mathfrak{a}_{n}=\sqrt{\mathfrak{a}_{n}}$ for all $n \in \mathbb{N}_{0}$. Finally, note that, when this is the case, we have $\mathfrak{a}_{n}=\mathfrak{a}_{n+1}$ for each $n \in \mathbb{N}_{0}$, by 4.2(i).

(iv) If $\mathfrak{A}$ is $\mathfrak{P}$-primary, then, since $\mathfrak{p}=\phi_{n}^{-1}(\mathfrak{P})$ (by part (i)) and $\mathfrak{a}_{n}=\phi_{n}^{-1}(\mathfrak{A})$, it follows that $\mathfrak{a}_{n}$ is $\mathfrak{p}$-primary (for all $n \in \mathbb{N}_{0}$ ).

Conversely, suppose that $\mathfrak{a}_{n}$ is $\mathfrak{p}$-primary for all $i \in \mathbb{N}_{0}$. Then, by parts (i) and (ii), we have $\mathfrak{A} \subseteq \sqrt{\mathfrak{A}}=\mathfrak{P}$, so that $\mathfrak{A}$ is proper. Suppose that $a, b \in R$ are such that $x^{-i} a x^{i} x^{-j} b x^{j} \in \mathfrak{A}$ for some integers $i, j \in \mathbb{N}_{0}$. Then $x^{-i-j} f^{j}(a) f^{i}(b) x^{i+j} \in \mathfrak{A}$, so that $f^{j}(a) f^{i}(b) \in \mathfrak{a}_{i+j}$. It follows from this that either $f^{j}(a) \in \mathfrak{a}_{i+j}$ or $f^{i}(b) \in$ $\sqrt{\mathfrak{a}_{i+j}}=\mathfrak{p}$, that is, either $a \in f^{-j}\left(\mathfrak{a}_{i+j}\right)=\mathfrak{a}_{i}$ or $b \in \mathfrak{p}$. Hence either $x^{-i} a x^{i} \in \mathfrak{A}$ or $x^{-j} b x^{j} \in \mathfrak{P}=\sqrt{\mathfrak{A}}$. Thus $\mathfrak{A}$ is $\mathfrak{P}$-primary.

(v) The $f$-sequence corresponding to the ideal $\bigcap_{\lambda \in \Lambda} \mathfrak{A}_{\lambda}$ of $R^{\infty}$ is, by 4.3 ,

$$
\left(\phi_{n}^{-1}\left(\bigcap_{\lambda \in \Lambda} \mathfrak{A}_{\lambda}\right)\right)_{n \in \mathbb{N}_{0}}=\left(\bigcap_{\lambda \in \Lambda} \phi_{n}^{-1}\left(\mathfrak{A}_{\lambda}\right)\right)_{n \in \mathbb{N}_{0}}=\left(\bigcap_{\lambda \in \Lambda} \mathfrak{a}_{\lambda, n}\right)_{n \in \mathbb{N}_{0}} .
$$

The final claim now follows from 4.3

We can quickly deduce from Theorem 6.1 that $R^{\infty}$ is only Noetherian in rather uninteresting cases.

6.2. Lemma. Let $\mathfrak{P} \in \operatorname{Spec}\left(R^{\infty}\right)$. Then $\mathfrak{P}^{\left[p^{n}\right]}=\mathfrak{P}^{n}=\mathfrak{P}$ for all $n \in \mathbb{N}$.

Proof. Let $\mathfrak{p} \in \operatorname{Spec}(R)$ correspond to $\mathfrak{P}$ in the correspondence of $6.1(\mathrm{i})$. Let $\alpha \in \mathfrak{P}$. With the notation of 4.3, we have $\alpha=x^{-i} \bar{r} x^{i}$ for some $r \in \mathfrak{p}$ and some $i \in \mathbb{N}_{0}$. Now $x^{-i-1} \bar{r} x^{i+1} \in x^{-i-1} \pi(\mathfrak{p}) x^{i+1} \subseteq \mathfrak{P}$. Hence $\alpha=x^{-i} \bar{r} x^{i}=x^{-i-1} \bar{r}^{p} x^{i+1}=$ 
$\left(x^{-i-1} \bar{r} x^{i+1}\right)^{p} \in \mathfrak{P}^{[p]}$. Hence $\mathfrak{P} \subseteq \mathfrak{P}^{[p]}$. It follows that $\mathfrak{P}=\mathfrak{P}^{[p]}$, and then, by induction, that $\mathfrak{P}=\mathfrak{P}^{\left[p^{n}\right]}$ for all $n \in \mathbb{N}$.

Finally, $\mathfrak{P} \supseteq \mathfrak{P}^{n} \supseteq \mathfrak{P}^{\left[p^{n}\right]}=\mathfrak{P}$ for all $n \in \mathbb{N}$.

We next justify our claim that $R^{\infty}$ is only Noetherian in uninteresting cases.

6.3. Theorem. The following conditions are equivalent:

(i) $R^{\infty}$ is Noetherian;

(ii) $R_{\text {red }}$ is a direct product of a finite number of fields;

(iii) $R^{\infty}$ is a direct product of a finite number of fields.

Proof. We can, and do, assume that $R$ is reduced.

(i) $\Rightarrow$ (ii) As $R^{\infty}$ is Noetherian, every prime ideal of $R^{\infty}$ is finitely generated. As for every prime ideal $\mathfrak{P}$ of $R^{\infty}$ we have $\mathfrak{P}^{2}=\mathfrak{P}$ (by 6.2), Nakayama's Lemma implies that each prime ideal in $R^{\infty}$ is a minimal prime. Hence $\operatorname{dim} R^{\infty}=0$. Therefore $R^{\infty}$ is Artinian with finitely many maximal ideals, $\mathfrak{M}_{1}, \ldots, \mathfrak{M}_{n}$, say. By Theorem 6.1(i), Spec $R=\left\{\mathfrak{m}_{1}, \ldots, \mathfrak{m}_{n}\right\}$, where $\mathfrak{m}_{i}$ corresponds to $\mathfrak{M}_{i}$ for $i=1, \ldots, n$ and each $\mathfrak{m}_{i}(1 \leq i \leq n)$ is a maximal ideal of $R$. As $R$ is reduced, the natural ring homomorphism $R \longrightarrow R / \mathfrak{m}_{1} \times \cdots \times R / \mathfrak{m}_{n}$ is an isomorphism.

(ii) $\Rightarrow$ (iii) This follows from 1.3 and 9 , Proposition 3.1].

(iii) $\Rightarrow$ (i) This is clear.

Thus it is not clear whether a given proper ideal $\mathfrak{A}$ of $R^{\infty}$ has a primary decomposition (although we shall present in $\$ 8$ some examples of proper ideals in non-Noetherian perfect closures that do have primary decompositions). But if it does, we can draw some interesting conclusions, as we now show.

6.4. Theorem. Let $\mathfrak{A}$ be an ideal of $R^{\infty}$ and let $\left(\mathfrak{a}_{n}\right)_{n \in \mathbb{N}_{0}}$ be the corresponding $f$-sequence of ideals of $R$. For each $k=1, \ldots, t$, let $\mathfrak{P}_{k}$ be a prime ideal of $R^{\infty}$, let $\mathfrak{p}_{k}$ be the corresponding prime ideal of $R$ (see 6.1(i)), let $\mathfrak{Q}_{k}$ be an ideal of $R^{\infty}$, and let $\left(\mathfrak{q}_{k, n}\right)_{n \in \mathbb{N}_{0}}$ be the $f$-sequence of ideals of $R$ corresponding to $\mathfrak{Q}_{k}$.

Then the following are equivalent:

(i) $\mathfrak{A}$ has a primary decomposition $\mathfrak{A}=\mathfrak{Q}_{1} \cap \cdots \cap \mathfrak{Q}_{t}$, where $\mathfrak{Q}_{k}$ is $\mathfrak{P}_{k}$-primary for $k=1, \ldots, t$

(ii) each $\mathfrak{a}_{n}$ has a primary decomposition $\mathfrak{a}_{n}=\mathfrak{q}_{1, n} \cap \cdots \cap \mathfrak{q}_{t, n}$ such that $\mathfrak{q}_{k, n}$ is $\mathfrak{p}_{k}$-primary for all $k=1, \ldots, t$ and all $n \in \mathbb{N}_{0}$.

Furthermore, when these equivalent conditions are satisfied,

$$
\bigcup_{n \in \mathbb{N}_{0}} \text { ass } \mathfrak{a}_{n}=\left\{\pi^{-1}\left(\mathfrak{P} \cap R_{\text {red }}\right): \mathfrak{P} \in \operatorname{ass}_{R^{\infty}}(\mathfrak{A})\right\}=\operatorname{ass} \mathfrak{a}_{j} \quad \text { for all } j \gg 0,
$$

and so is finite; also, the primary decomposition of $\mathfrak{A}$ given in (i) is minimal if and only if the primary decomposition of $\mathfrak{a}_{n}$ given in (ii) is minimal for all $n \gg 0$.

Proof. Assume that statement (i) is true. By 6.1(iv), $\mathfrak{q}_{k, n}$ is $\mathfrak{p}_{k}$-primary for all $k=1, \ldots, t$ and all $n \in \mathbb{N}_{0}$; furthermore, with the notation of 4.3 , we have, for each $n \in \mathbb{N}_{0}$,

$$
\mathfrak{a}_{n}=\psi_{n}^{-1}(\mathfrak{A})=\psi_{n}^{-1}\left(\bigcap_{k=1}^{t} \mathfrak{Q}_{k}\right)=\bigcap_{k=1}^{t} \psi_{n}^{-1}\left(\mathfrak{Q}_{k}\right)=\bigcap_{k=1}^{t} \mathfrak{q}_{k, n} .
$$

Thus statement (ii) is true. 
Now suppose, in addition, that $\mathfrak{A}=\mathfrak{Q}_{1} \cap \cdots \cap \mathfrak{Q}_{t}$ is a minimal primary decomposition of $\mathfrak{A}$. The bijective correspondence of 6.1(i) shows that $\mathfrak{p}_{1}, \ldots, \mathfrak{p}_{t}$ are all distinct. Choose $j \in\{1, \ldots, t\}$. Since (in view of $6.1(\mathrm{v})$ )

$$
\bigcup_{n \in \mathbb{N}_{0}} x^{-n} \pi\left(\mathfrak{q}_{j, n}\right) x^{n}=\mathfrak{Q}_{j} \nsupseteq \bigcap_{\substack{k=1 \\ k \neq j}}^{t} \mathfrak{Q}_{k}=\bigcup_{n \in \mathbb{N}_{0}}\left(x^{-n} \pi\left(\bigcap_{\substack{k=1 \\ k \neq j}}^{t} \mathfrak{q}_{k, n}\right) x^{n}\right),
$$

there exists $n \in \mathbb{N}_{0}$ such that

$$
\mathfrak{q}_{j, n} \nsupseteq \bigcap_{\substack{k=1 \\ k \neq j}}^{t} \mathfrak{q}_{k, n} .
$$

Hence refinement of the primary decomposition $\mathfrak{a}_{n}=\mathfrak{q}_{1, n} \cap \cdots \cap \mathfrak{q}_{t, n}$ to a minimal primary decomposition cannot result in the removal of $\mathfrak{q}_{j, n}$. Therefore $\mathfrak{p}_{j} \in$ ass $\mathfrak{a}_{n}$. Since the sequence (ass $\left.\mathfrak{a}_{n}\right)_{n \in \mathbb{N}_{0}}$ is increasing (by 4.2 (iv)), it follows that $\mathfrak{p}_{j} \in$ ass $\mathfrak{a}_{n+h}$ for all $h \in \mathbb{N}_{0}$. It follows that the primary decomposition of $\mathfrak{a}_{m}$ given in (ii) is minimal for all $m \gg 0$.

Now assume that statement (ii) is true. It follows from 6.1(iv) that $\mathfrak{Q}_{k}$ is $\mathfrak{P}_{k^{-}}$ primary, and from 6.1(v) that

$$
\begin{aligned}
\mathfrak{A} & =\bigcup_{n \in \mathbb{N}_{0}} x^{-n} \pi\left(\mathfrak{a}_{n}\right) x^{n}=\bigcup_{n \in \mathbb{N}_{0}}\left(x^{-n} \pi\left(\bigcap_{k=1}^{t} \mathfrak{q}_{k, n}\right) x^{n}\right) \\
& =\bigcap_{k=1}^{t}\left(\bigcup_{n \in \mathbb{N}_{0}} x^{-n} \pi\left(\mathfrak{q}_{k, n}\right) x^{n}\right)=\bigcap_{k=1}^{t} \mathfrak{Q}_{k} .
\end{aligned}
$$

Thus statement (i) is true.

Now suppose that the primary decomposition of $\mathfrak{a}_{j}$ given in (ii) is minimal for all $j \gg 0$. This means that $\mathfrak{p}_{1}, \ldots, \mathfrak{p}_{t}$ are all different (so that $\mathfrak{P}_{1}, \ldots, \mathfrak{P}_{t}$ are all different) and ass $\mathfrak{a}_{j}=\left\{\mathfrak{p}_{1}, \ldots, \mathfrak{p}_{t}\right\}$ for all $j \gg 0$. Now if the primary decomposition $\mathfrak{A}=\bigcap_{k=1}^{t} \mathfrak{Q}_{k}$ were not minimal, then it would be possible to refine it to a minimal primary decomposition which would have fewer than $t$ terms; it would then follow from what we have proved above (when dealing with the implication (i) $\Rightarrow$ (ii)) that all the sets ass $\mathfrak{a}_{n}\left(n \in \mathbb{N}_{0}\right)$ would have fewer than $t$ terms, and this would be a contradiction. Hence $\mathfrak{A}=\bigcap_{k=1}^{t} \mathfrak{Q}_{k}$ must be a minimal primary decomposition.

It follows from Theorem 6.3 that, provided $R_{\text {red }}$ is not a direct product of a finite number of fields, there will be ideals in $R^{\infty}$ that are not finitely generated. It will be helpful to have information about exactly when an ideal of $R^{\infty}$ is finitely generated, and our next result provides this. Its proof uses some ideas from the proof of Jordan 9, Theorem 5.6]. Observe that if $\mathfrak{A}=\left(\alpha_{1}, \ldots, \alpha_{t}\right) R^{\infty}$ is a finitely generated ideal of $R^{\infty}$, then there exist $k \in \mathbb{N}_{0}$ and $a_{1}, \ldots, a_{t} \in R$ such that $\alpha_{j}=x^{-k} \overline{a_{j}} x^{k}$ for all $j=1, \ldots, t$.

6.5. Theorem. Let $\mathfrak{A} \subseteq R^{\infty}$ be an ideal and $\left(\mathfrak{a}_{n}\right)_{n \in \mathbb{N}_{0}}$ be the $f$-sequence corresponding to $\mathfrak{A}$. Let $k \in \mathbb{N}_{0}$ and $a_{1}, \ldots, a_{t} \in R$. Then, with the notation of 4.3 , the following statements are equivalent:

(i) $\mathfrak{A}=\left(x^{-k} \overline{a_{1}} x^{k}, \ldots, x^{-k} \overline{a_{t}} x^{k}\right) R^{\infty}$;

(ii) $\mathfrak{a}_{k+n}=\left(\left(a_{1}, \ldots, a_{t}\right)^{\left[p^{n}\right]}\right)^{F}$ for all $n \in \mathbb{N}_{0}$. 
Note. When statements (i) and (ii) in the theorem are satisfied, we can describe in precise terms not only the $\mathfrak{a}_{k+n}$ for $n \in \mathbb{N}_{0}$, but also each $\mathfrak{a}_{j}$ for $0 \leq j<k$ : since $\left(\mathfrak{a}_{n}\right)_{n \in \mathbb{N}_{0}}$ is an $f$-sequence, we must have $\mathfrak{a}_{j}=f^{-(k-j)}\left(\mathfrak{a}_{k}\right)=f^{-(k-j)}\left(\left(a_{1}, \ldots, a_{t}\right)^{F}\right)$.

Note also that a special case of this theorem yields that, if $\mathfrak{a}$ is an ideal of $R$, then the $f$-sequence that corresponds to the ideal $\mathfrak{a} R^{\infty}$ of $R^{\infty}$ is $\left(\left(\mathfrak{a}^{\left[p^{n}\right]}\right)^{F}\right)_{n \in \mathbb{N}_{0}}$.

Proof. (i) $\Rightarrow$ (ii) Let $n \in \mathbb{N}_{0}$. As $x^{-k} \overline{a_{j}} x^{k} \in \mathfrak{A}$, we have $a_{j} \in \mathfrak{a}_{k}$ for all $j=1, \ldots, t$, and so it follows from 4.2(i) that $\left(\left(a_{1}, \ldots, a_{t}\right)^{\left[p^{n}\right]}\right)^{F} \subseteq \mathfrak{a}_{k+n}$.

Next consider $b \in \mathfrak{a}_{k+n}$. Then, by definition, $x^{-(k+n)} \bar{b} x^{k+n} \in \mathfrak{A}$. As $\mathfrak{A}=$ $\left(x^{-k} \overline{a_{1}} x^{k}, \ldots, x^{-k} \overline{a_{t}} x^{k}\right) R^{\infty}$, we can write $x^{-(k+n)} \bar{b} x^{k+n}=\sum_{j=1}^{t} \rho_{j} x^{-k} \overline{a_{j}} x^{k}$ for some $\rho_{1}, \ldots, \rho_{t} \in R^{\infty}$. Hence

$$
\bar{b}=\sum_{j=1}^{t} \rho_{j}^{p^{k+n}} x^{-k} \overline{a_{j}} p^{k+n} x^{k}=\sum_{j=1}^{t} \rho_{j}^{p^{k+n}} \overline{a_{j}} p^{n} .
$$

As $\rho_{1}, \ldots, \rho_{t} \in R^{\infty}$, there exists $m \in \mathbb{N}_{0}$ such that, for all $j=1, \ldots, t$, we have $\rho_{j}^{p^{k+n+m}} \in R_{\text {red }}$. Therefore $\bar{b}^{p^{m}}=\sum_{j=1}^{t} \rho_{j}^{p^{k+n+m}}{\overline{a_{j}}}^{p^{n+m}} \in\left(\overline{a_{1}}, \ldots, \overline{a_{t}}\right)^{\left[p^{n+m}\right]}$. Hence there exists $b^{\prime} \in\left(a_{1}, \ldots, a_{t}\right)^{\left[p^{n+m}\right]}$ such that $b^{p^{m}}-b^{\prime} \in \sqrt{(0)}$, and it follows easily that $b \in\left(\left(a_{1}, \ldots, a_{t}\right)^{\left[p^{n}\right]}\right)^{F}$. Therefore

$$
\mathfrak{a}_{k+n} \subseteq\left(\left(a_{1}, \ldots, a_{t}\right)^{\left[p^{n}\right]}\right)^{F} .
$$

(ii) $\Rightarrow$ (i) As $\mathfrak{a}_{k}=\left(a_{1}, \ldots, a_{t}\right)^{F}$ and $\mathfrak{A}=\bigcup_{n \in \mathbb{N}_{0}} x^{-n} \pi\left(\mathfrak{a}_{n}\right) x^{n}$, it follows that $x^{-k} \overline{a_{1}} x^{k}, \ldots, x^{-k} \overline{a_{t}} x^{k} \in \mathfrak{A}$. Hence $\left(x^{-k} \overline{a_{1}} x^{k}, \ldots, x^{-k} \overline{a_{t}} x^{k}\right) R^{\infty} \subseteq \mathfrak{A}$.

Next consider $\gamma \in \mathfrak{A}$. We can write $\gamma=x^{-i} \bar{a} x^{i}$ for some $i \in \overline{\mathbb{N}}_{0}$ and some $a \in \mathfrak{a}_{i}$. If $i<k$, then $\gamma=x^{-k} \overline{a^{p^{k-i}}} x^{k}$ with $a^{p^{k-i}} \in \mathfrak{a}_{k}$. Hence we can assume that $i \geq k$. Write $i=k+n$, where $n \in \mathbb{N}_{0}$.

Then, as $a \in \mathfrak{a}_{k+n}=\left(\left(a_{1}, \ldots, a_{t}\right)^{\left[p^{n}\right]}\right)^{F}$, there exists $m \in \mathbb{N}_{0}$ such that

$$
a^{p^{m}} \in\left(\left(a_{1}, \ldots, a_{t}\right)^{\left[p^{n}\right]}\right)^{\left[p^{m}\right]}=\left(a_{1}, \ldots, a_{t}\right)^{\left[p^{n+m}\right]} .
$$

Therefore, we can write $a^{p^{m}}=\sum_{j=1}^{t} r_{j} a_{j}^{p^{n+m}}$ for some $r_{1}, \ldots, r_{t} \in R$. Hence

$$
\begin{aligned}
\gamma^{p^{m}} & =\left(x^{-(k+n)} \bar{a} x^{k+n}\right)^{p^{m}}=x^{-(k+n)} \bar{a}^{p^{m}} x^{k+n}=x^{-(k+n)}\left(\sum_{j=1}^{t} \overline{r_{j} a_{j}^{p^{n+m}}}\right) x^{k+n} \\
& =\sum_{j=1}^{t} x^{-(k+n)} \overline{r_{j}} x^{k+n} x^{-(k+n)}{\overline{a_{j}}}^{n+m} x^{k+n} \\
& =\sum_{j=1}^{t} x^{-(k+n+m)} \overline{r_{j}} p^{m} x^{k+n+m} x^{-k}{\overline{a_{j}}}^{p^{m}} x^{k} \\
& =\left(\sum_{j=1}^{t} x^{-(k+n+m)} \overline{r_{j}} x^{k+n+m} x^{-k} \overline{a_{j}} x^{k}\right)^{p^{m}} .
\end{aligned}
$$

Therefore $\gamma=\sum_{j=1}^{t} x^{-(k+n+m)} \overline{r_{j}} x^{k+n+m} x^{-k} \overline{a_{j}} x^{k}$ because the Frobenius homomorphism acts bijectively on $R^{\infty}$; hence $\gamma \in\left(x^{-k} \overline{a_{1}} x^{k}, \ldots, x^{-k} \overline{a_{t}} x^{k}\right) R^{\infty}$. 


\section{LINEAR GROWTH OF PRIMARY DECOMPOSITIONS}

Throughout this section, $R$ will denote a commutative Noetherian ring of prime characteristic $p$. In the following definition, we extend the concept of 'linear growth of primary decompositions', defined in the Introduction for the sequence of Frobenius powers of a given ideal of $R$, to $f$-sequences.

7.1. Definition. Let $\left(\mathfrak{a}_{n}\right)_{n \in \mathbb{N}_{0}}$ be an $f$-sequence of proper ideals of $R$ and let $h$ be a positive integer.

We say that $\left(\mathfrak{a}_{n}\right)_{n \in \mathbb{N}_{0}}$ has $h$-linear growth of primary decompositions if, for every non-negative integer $n$, there exists a minimal primary decomposition

$$
\mathfrak{a}_{n}=\mathfrak{q}_{1, n} \cap \cdots \cap \mathfrak{q}_{k_{n}, n} \quad \text { with }{\sqrt{\mathfrak{q}_{i, n}}}^{\left[p^{n}\right] h} \subseteq \mathfrak{q}_{i, n} \text { for all } i=1, \ldots, k_{n} .
$$

We say that $\left(\mathfrak{a}_{n}\right)_{n \in \mathbb{N}_{0}}$ has linear growth of primary decompositions if it has $k$-linear growth of primary decompositions for some positive integer $k$.

Let $\mathfrak{a}$ be a proper ideal of $R$. Although the sequence of Frobenius powers of $\mathfrak{a}$ need not be an $f$-sequence, we say that the Frobenius powers of $\mathfrak{a}$ have $h$-linear growth of primary decompositions if, for every non-negative integer $n$, there exists a minimal primary decomposition

$$
\mathfrak{a}^{\left[p^{n}\right]}=\mathfrak{q}_{1, n} \cap \cdots \cap \mathfrak{q}_{k_{n}, n} \quad \text { with }{\sqrt{\mathfrak{q}_{i, n}}}^{\left[p^{n}\right] h} \subseteq \mathfrak{q}_{i, n} \text { for all } i=1, \ldots, k_{n} .
$$

An elementary argument will prove the following lemma.

7.2. Lemma. Let $\eta: R \longrightarrow R^{\prime}$ be a homomorphism of commutative Noetherian rings of characteristic $p$, and let $\left(\mathfrak{a}_{n}^{\prime}\right)_{n \in \mathbb{N}_{0}}$ be an $f$-sequence of ideals of $R^{\prime}$. Let $\eta^{\infty}: R^{\infty} \longrightarrow R^{\prime \infty}$ be the ring homomorphism induced by $\eta$ (see $\S 1$ ). By 4.4. the sequence $\left(\eta^{-1}\left(\mathfrak{a}_{n}^{\prime}\right)\right)_{n \in \mathbb{N}_{0}}$ is an $f$-sequence of ideals of $R$.

If $\left(\mathfrak{a}_{n}^{\prime}\right)_{n \in \mathbb{N}_{0}}$ has $h$-linear growth of primary decompositions for some $h \in \mathbb{N}$, then $\left(\eta^{-1}\left(\mathfrak{a}_{n}^{\prime}\right)\right)_{n \in \mathbb{N}_{0}}$ also has h-linear growth of primary decompositions.

Several of the results in this section will involve the hypothesis that $R$ has a $p^{m_{0}}$-weak test element for some $m_{0} \in \mathbb{N}_{0}$. We remind the reader of the definition of such elements.

7.3. Definition (See [3, $\S 6]$ ). Let $m_{0} \in \mathbb{N}_{0}$. A $p^{m_{0}}$-weak test element for $R$ is an element $c \in R^{\circ}$ such that, for every ideal $\mathfrak{b}$ of $R$ and for $r \in R$, it is the case that $r \in \mathfrak{b}^{*}$ if and only if $c r^{p^{n}} \in \mathfrak{b}^{\left[p^{n}\right]}$ for all $n \geq m_{0}$. A $p^{0}$-weak test element for $R$ is called a test element for $R$.

Such a $p^{m_{0}}$-weak test element $c$ for $R$ is said to be a locally stable $p^{m_{0}}$-weak test element for $R$ if, for each $\mathfrak{p} \in \operatorname{Spec}(R)$, the image of $c$ in the localization $R_{\mathfrak{p}}$ is a $p^{m_{0}}$-weak test element for $R_{\mathfrak{p}}$.

Also, a $p^{m_{0}}$-weak test element $c$ for $R$ is said to be a completely stable $p^{m_{0}}$-weak test element for $R$ if it is locally stable and, for each $\mathfrak{p} \in \operatorname{Spec}(R)$, the image of $c$ in the completion $\widehat{R_{\mathfrak{p}}}$ of the localization of $R$ at $\mathfrak{p}$ is a $p^{m_{0}}$-weak test element for $\widehat{R_{\mathfrak{p}}}$.

It is a result of Hochster and Huneke [5, Theorem $(6.1)(b)]$ that an algebra of finite type over an excellent local ring of characteristic $p$ has a completely stable $p^{m_{0}}$-weak test element, for some $m_{0} \in \mathbb{N}_{0}$.

7.4. Lemma. Let $\eta: R \longrightarrow R^{\prime}$ be a homomorphism of commutative Noetherian rings of characteristic $p$, let $S$ be a multiplicatively closed subset of $R$, and let $e$ stand for extension with respect to the natural ring homomorphism $R \longrightarrow S^{-1} R$.

(i) If $\mathfrak{a}^{\prime}$ is an F-closed ideal of $R^{\prime}$, then $\eta^{-1}\left(\mathfrak{a}^{\prime}\right)$ is an $F$-closed ideal of $R$. 
(ii) If $\mathfrak{a}^{\prime}$ is a tightly-closed ideal of $R^{\prime}$ and there exists a $p^{m_{0}}$-weak test element (where $m_{0} \in \mathbb{N}_{0}$ ) c for $R$ such that $\eta(c)$ is a $p^{m_{0}}$-weak test element for $R^{\prime}$, then $\eta^{-1}\left(\mathfrak{a}^{\prime}\right)$ is a tightly-closed ideal of $R$.

(iii) If $\mathfrak{a}$ is an F-closed ideal of $R$, then $\mathfrak{a}^{e}$ is an $F$-closed ideal of $S^{-1} R$.

Proof. (i) Let $r \in\left(\eta^{-1}\left(\mathfrak{a}^{\prime}\right)\right)^{F}$, so there exists $n \in \mathbb{N}_{0}$ such that $r^{p^{n}} \in\left(\eta^{-1}\left(\mathfrak{a}^{\prime}\right)\right)^{\left[p^{n}\right]}$. Thus there exist $a_{1}, \ldots, a_{t} \in \eta^{-1}\left(\mathfrak{a}^{\prime}\right)$ and $r_{1}, \ldots, r_{t} \in R$ such that

$$
r^{p^{n}}=r_{1} a_{1}^{p^{n}}+\cdots+r_{t} a_{t}^{p^{n}}
$$

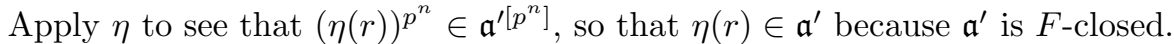

(ii) Let $r \in\left(\eta^{-1}\left(\mathfrak{a}^{\prime}\right)\right)^{*}$, so that $c r^{p^{n}} \in\left(\eta^{-1}\left(\mathfrak{a}^{\prime}\right)\right)^{\left[p^{n}\right]}$ for all $n \geq m_{0}$. Application of $\eta$ then yields that $\eta(c) \eta(r)^{p^{n}} \in \mathfrak{a}^{\prime\left[p^{n}\right]}$ for all $n \geq m_{0}$. Since $\eta(c)$ is a $p^{m_{0}}$-weak test element for $R^{\prime}$ and $\mathfrak{a}^{\prime}$ is tightly closed, $\eta(r) \in \mathfrak{a}^{\prime}$.

(iii) Let $r \in R$ be such that $r / 1 \in\left(\mathfrak{a}^{e}\right)^{F}$ in $S^{-1} R$, so that there exists $n \in \mathbb{N}_{0}$ with $r^{p^{n}} / 1 \in\left(\mathfrak{a}^{e}\right)^{\left[p^{n}\right]}=\left(\mathfrak{a}^{\left[p^{n}\right]}\right)^{e}$. Thus there exists $s \in S$ such that $s r^{p^{n}} \in \mathfrak{a}^{\left[p^{n}\right]}$; since $s r \in \mathfrak{a}^{F}=\mathfrak{a}$, it follows that $r / 1=s r / s \in \mathfrak{a}^{e}$.

The motivation for our study of linear growth of primary decompositions came from work of K. E. Smith and I. Swanson: in [15, Theorem (1.2)], they showed that, in $K\left[X_{1}, \ldots, X_{d}\right] / \mathfrak{b}$, where $K$ is a field of characteristic $p, X_{1}, \ldots, X_{d}$ are indeterminates, and $\mathfrak{b}$ is an ideal of the polynomial ring $K\left[X_{1}, \ldots, X_{d}\right]$ generated by monomials, the Frobenius powers of a proper ideal $\mathfrak{a}$ generated by monomials in the images of $X_{1}, \ldots, X_{d}$ have linear growth of primary decompositions (in the sense of Definition [7.1), and $\bigcup_{n \in \mathbb{N}_{0}}$ ass $\mathfrak{a}^{\left[p^{n}\right]}$ is a finite set.

Our first major result in this section demonstrates a connection between the two definitions in 7.1 it shows that, if the Frobenius powers of the proper ideal $\mathfrak{a}$ of $R$ have $h$-linear growth of primary decompositions and $\bigcup_{n \in \mathbb{N}_{0}}$ ass $\mathfrak{a}^{\left[p^{n}\right]}$ is finite, then the canonical $f$-sequence $\left(\left(\mathfrak{a}^{\left[p^{n}\right]}\right)^{F}\right)_{n \in \mathbb{N}_{0}}$ associated to $\mathfrak{a}$ (see 4.1(iii)) has $h$ linear growth of primary decompositions. However, this result should come with the warning that M. Katzman [10] has provided an example of a proper ideal $\mathfrak{d}$ in a ring of the type under consideration here for which $\bigcup_{n \in \mathbb{N}_{0}}$ ass $\mathfrak{d}^{\left[p^{n}\right]}$ is infinite.

7.5. Theorem. Suppose that the Frobenius powers of the proper ideal a of $R$ have $h$ linear growth of primary decompositions (for some $h \in \mathbb{N}$ ), and that $\bigcup_{n \in \mathbb{N}_{0}}$ ass $\mathfrak{a}^{\left[p^{n}\right]}$ is finite; let the members of the latter set be $\mathfrak{p}_{1}, \ldots, \mathfrak{p}_{t}$, and let the corresponding prime ideals of $R^{\infty}$ (see $6.1(\mathrm{i})$ ) be $\mathfrak{P}_{1}, \ldots, \mathfrak{P}_{t}$, respectively.

For each $i=1, \ldots, t$, let ${ }^{e_{i}}$ and ${ }^{c_{i}}$ stand for extension and contraction with respect to the natural ring homomorphism $R \longrightarrow R_{\mathfrak{p}_{i}}$, and let ${ }^{E_{i}}$ and $C_{i}$ stand for extension and contraction with respect to the natural ring homomorphism $R^{\infty} \longrightarrow$ $\left(R^{\infty}\right)_{\mathfrak{P}_{i}}$

(i) For each $n \in \mathbb{N}_{0}$,

$$
\mathfrak{a}^{\left[p^{n}\right]}=\bigcap_{i=1}^{t}\left(\left(\mathfrak{a}+\mathfrak{p}_{i}^{h}\right)^{\left[p^{n}\right]}\right)^{e_{i} c_{i}}
$$

is a primary decomposition, where $\left(\left(\mathfrak{a}+\mathfrak{p}_{i}^{h}\right)^{\left[p^{n}\right]}\right)^{e_{i} c_{i}}$ is $\mathfrak{p}_{i}$-primary for $i=$ $1, \ldots, t$. 
(ii) For each $n \in \mathbb{N}_{0}$,

$$
\left(\mathfrak{a}^{\left[p^{n}\right]}\right)^{F}=\bigcap_{i=1}^{t}\left(\left(\left(\mathfrak{a}+\mathfrak{p}_{i}^{h}\right)^{\left[p^{n}\right]}\right)^{F}\right)^{e_{i} c_{i}}
$$

is a primary decomposition in which all the primary components are $F$ closed, where $\left(\left(\left(\mathfrak{a}+\mathfrak{p}_{i}^{h}\right)^{\left[p^{n}\right]}\right)^{F}\right)^{e_{i} c_{i}}$ is $\mathfrak{p}_{i}$-primary for $i=1, \ldots, t$.

(iii) The $f$-sequence $\left(\left(\mathfrak{a}^{\left[p^{n}\right]}\right)^{F}\right)_{n \in \mathbb{N}_{0}}$ has h-linear growth of primary decompositions.

(iv) We have $\bigcup_{n \in \mathbb{N}_{0}} \operatorname{ass}\left(\mathfrak{a}^{\left[p^{n}\right]}\right)^{F} \subseteq \bigcup_{n \in \mathbb{N}_{0}}$ ass $\mathfrak{a}^{\left[p^{n}\right]}$, and so $\bigcup_{n \in \mathbb{N}_{0}} \operatorname{ass}\left(\mathfrak{a}^{\left[p^{n}\right]}\right)^{F}$ is finite.

(v) Furthermore,

$$
\mathfrak{a} R^{\infty}=\bigcap_{i=1}^{t}\left(\left(\mathfrak{a}+\mathfrak{p}_{i}^{h}\right) R^{\infty}\right)^{E_{i} C_{i}}
$$

is a primary decomposition of the ideal $\mathfrak{a} R^{\infty}$ of $R^{\infty}$ to which the $f$-sequence $\left(\left(\mathfrak{a}{ }^{\left[p^{n}\right]}\right)^{F}\right)_{n \in \mathbb{N}_{0}}$ corresponds, where $\left(\left(\mathfrak{a}+\mathfrak{p}_{i}^{h}\right) R^{\infty}\right)^{E_{i} C_{i}}$ is $\mathfrak{P}_{i}$-primary for $i=$ $1, \ldots, t$.

Proof. For each $n \in \mathbb{N}_{0}$, there exists a subset $\Lambda_{n}$ of $\{1, \ldots, t\}$ and a minimal primary decomposition $\mathfrak{a}^{\left[p^{n}\right]}=\bigcap_{i \in \Lambda_{n}} \mathfrak{q}_{i, n}$, where $\mathfrak{q}_{i, n}$ is $\mathfrak{p}_{i}$-primary and $\mathfrak{p}_{i}^{h\left[p^{n}\right]} \subseteq \mathfrak{q}_{i, n}$ for all $i \in \Lambda_{n}$.

(i) Now $\mathfrak{a}^{\left[p^{n}\right]} \subseteq \mathfrak{a}^{\left[p^{n}\right]}+\mathfrak{p}_{i}^{h\left[p^{n}\right]}=\left(\mathfrak{a}+\mathfrak{p}_{i}^{h}\right)^{\left[p^{n}\right]} \subseteq \mathfrak{q}_{i, n}$, so that

$$
\mathfrak{a}^{\left[p^{n}\right]} \subseteq\left(\left(\mathfrak{a}+\mathfrak{p}_{i}^{h}\right)^{\left[p^{n}\right]}\right)^{e_{i} c_{i}} \subseteq\left(\mathfrak{q}_{i, n}\right)^{e_{i} c_{i}}=\mathfrak{q}_{i, n} \quad \text { for all } i \in \Lambda_{n}
$$

Hence $\mathfrak{a}^{\left[p^{n}\right]}=\bigcap_{i \in \Lambda_{n}}\left(\left(\mathfrak{a}+\mathfrak{p}_{i}^{h}\right)^{\left[p^{n}\right]}\right)^{e_{i} c_{i}} ;$ moreover, for all $i \in\{1, \ldots, t\}$, we have $\mathfrak{p}_{i}^{h\left[p^{n}\right]} \subseteq\left(\mathfrak{a}+\mathfrak{p}_{i}^{h}\right)^{\left[p^{n}\right]} \subseteq \mathfrak{p}_{i}$, so that $\left(\left(\mathfrak{a}+\mathfrak{p}_{i}^{h}\right)^{\left[p^{n}\right]}\right)^{e_{i} c_{i}}$ is $\mathfrak{p}_{i}$-primary. It follows that

$$
\mathfrak{a}^{\left[p^{n}\right]}=\bigcap_{i=1}^{t}\left(\left(\mathfrak{a}+\mathfrak{p}_{i}^{h}\right)^{\left[p^{n}\right]}\right)^{e_{i} c_{i}}
$$

is a primary decomposition, where $\left(\left(\mathfrak{a}+\mathfrak{p}_{i}^{h}\right)^{\left[p^{n}\right]}\right)^{e_{i} c_{i}}$ is $\mathfrak{p}_{i}$-primary for $i=1, \ldots, t$.

(ii), (iii), (iv) It is clear that

$$
\left(\mathfrak{a}^{\left[p^{n}\right]}\right)^{F} \subseteq \bigcap_{i=1}^{t}\left(\left(\mathfrak{a}+\mathfrak{p}_{i}^{h}\right)^{\left[p^{n}\right]}\right)^{F} \subseteq \bigcap_{i=1}^{t}\left(\left(\left(\mathfrak{a}+\mathfrak{p}_{i}^{h}\right)^{\left[p^{n}\right]}\right)^{F}\right)^{e_{i} c_{i}} .
$$

Note that, for each $i \in\{1, \ldots, t\}$, we have $\mathfrak{p}_{i}^{h\left[p^{n}\right]} \subseteq\left(\mathfrak{a}+\mathfrak{p}_{i}^{h}\right)^{\left[p^{n}\right]} \subseteq \mathfrak{p}_{i}$, so that

$$
\mathfrak{p}_{i}^{h\left[p^{n}\right]} \subseteq\left(\left(\mathfrak{a}+\mathfrak{p}_{i}^{h}\right)^{\left[p^{n}\right]}\right)^{F} \subseteq\left(\left(\left(\mathfrak{a}+\mathfrak{p}_{i}^{h}\right)^{\left[p^{n}\right]}\right)^{F}\right)^{e_{i} c_{i}} \subseteq\left(\mathfrak{p}_{i}^{F}\right)^{e_{i} c_{i}}=\mathfrak{p}_{i}^{e_{i} c_{i}}=\mathfrak{p}_{i} .
$$

It follows that $\left(\left(\left(\mathfrak{a}+\mathfrak{p}_{i}^{h}\right)^{\left[p^{n}\right]}\right)^{F}\right)^{e_{i} c_{i}}$ is $\mathfrak{p}_{i}$-primary and it is $F$-closed by 7.4 (i),(iii). Therefore, the proof of parts (ii), (iii) and (iv) will be completed as soon as it is shown that $\left(\mathfrak{a}^{\left[p^{n}\right]}\right)^{F} \supseteq \bigcap_{i=1}^{t}\left(\left(\left(\mathfrak{a}+\mathfrak{p}_{i}^{h}\right)^{\left[p^{n}\right]}\right)^{F}\right)^{e_{i} c_{i}}$ for each $n \in \mathbb{N}_{0}$.

So let $r \in \bigcap_{i=1}^{t}\left(\left(\left(\mathfrak{a}+\mathfrak{p}_{i}^{h}\right)^{\left[p^{n}\right]}\right)^{F}\right)^{e_{i} c_{i}}$. Then, for each $i \in\{1, \ldots, t\}$, there exists $s_{i} \in R \backslash \mathfrak{p}_{i}$ such that $s_{i} r \in\left(\left(\mathfrak{a}+\mathfrak{p}_{i}^{h}\right)^{\left[p^{n}\right]}\right)^{F}$; this means that there exists $k_{i} \in \mathbb{N}_{0}$ with $\left(s_{i} r\right)^{p^{k_{i}}} \in\left(\left(\mathfrak{a}+\mathfrak{p}_{i}^{h}\right)^{\left[p^{n}\right]}\right)^{\left[p^{k_{i}}\right]}$. Set $k:=\max \left\{k_{i}: i=1, \ldots, t\right\}$. Then $\left(s_{i} r\right)^{p^{k}} \in$ $\left(\left(\mathfrak{a}+\mathfrak{p}_{i}^{h}\right)^{\left[p^{n}\right]}\right)^{\left[p^{k}\right]}=\left(\mathfrak{a}+\mathfrak{p}_{i}^{h}\right)^{\left[p^{n+k}\right]}$, so that

$$
r^{p^{k}} \in\left(\left(\mathfrak{a}+\mathfrak{p}_{i}^{h}\right)^{\left[p^{n+k}\right]}\right)^{e_{i} c_{i}} \quad \text { for all } i=1, \ldots, t \text {. }
$$


Hence, using part (i), we see that $r^{p^{k}} \in \bigcap_{i=1}^{t}\left(\left(\mathfrak{a}+\mathfrak{p}_{i}^{h}\right)^{\left[p^{n+k}\right]}\right)^{e_{i} c_{i}}=\mathfrak{a}^{\left[p^{n+k}\right]}=$ $\left(\mathfrak{a}^{\left[p^{n}\right]}\right)^{\left[p^{k}\right]}$. Therefore $r \in\left(\mathfrak{a}^{\left[p^{n}\right]}\right)^{F}$, as required.

(v) For each $i=1, \ldots, t$, the sequence $\left(\left(\left(\mathfrak{a}+\mathfrak{p}_{i}^{h}\right)^{\left[p^{n}\right]}\right)^{F}\right)_{n \in \mathbb{N}_{0}}$ is the canonical $f$-sequence associated to $\mathfrak{a}+\mathfrak{p}_{i}^{h}$; see 4.1 (iii). This $f$-sequence corresponds to $\left(\mathfrak{a}+\mathfrak{p}_{i}^{h}\right) R^{\infty}$, by Theorem 6.5; similarly, $\mathfrak{a} R^{\infty}$ is the ideal of $R^{\infty}$ corresponding to the $f$-sequence $\left(\left(\mathfrak{a}^{\left[p^{n}\right]}\right)^{F}\right)_{n \in \mathbb{N}_{0}}$. It is easy to check that $\bigcup_{n \in \mathbb{N}_{0}} \psi_{n}\left(R \backslash \mathfrak{p}_{i}\right)=R^{\infty} \backslash \mathfrak{P}_{i}$, and so it follows from Lemma 5.5 that $\left(\left(\mathfrak{a}+\mathfrak{p}_{i}^{h}\right) R^{\infty}\right)^{E_{i} C_{i}}$ corresponds to the $f$-sequence $\left(\left(\left(\left(\mathfrak{a}+\mathfrak{p}_{i}^{h}\right)^{\left[p^{n}\right]}\right)^{F}\right)^{e_{i} c_{i}}\right)_{n \in \mathbb{N}_{0}}$. By part (ii), each term in the latter $f$-sequence is $\mathfrak{p}_{i}$-primary. The result therefore follows from Theorem 6.4

It was explained in the Introduction that our interest in linear growth of primary decompositions of Frobenius powers of a proper ideal $\mathfrak{a}$ of $R$ arose from the argument of Smith and Swanson in [15] that shows that if the Frobenius powers of $\mathfrak{a}$ have linear growth of primary decompositions, then $\mathfrak{a}^{*} R_{u}=\left(\mathfrak{a} R_{u}\right)^{*}$ for all $u \in R$. Our next result shows, among other things, that linear growth of primary decompositions of an $f$-sequence $\left(\mathfrak{a}_{n}\right)_{n \in \mathbb{N}_{0}}$ that approximates to the Frobenius powers of $\mathfrak{a}$ in the sense that $\mathfrak{a}^{\left[p^{n}\right]} \subseteq \mathfrak{a}_{n} \subseteq\left(\mathfrak{a}^{\left[p^{n}\right]}\right)^{*}$ for all $n \in \mathbb{N}_{0}$ would do just as well in this context, provided that $R$ has a $p^{m_{0}}$-weak test element, for some $m_{0} \in \mathbb{N}_{0}$. Note that Theorem 7.5 shows that if the Frobenius powers of $\mathfrak{a}$ have linear growth of primary decompositions, then, provided $\bigcup_{n \in \mathbb{N}_{0}}$ ass $\mathfrak{a}^{\left[p^{n}\right]}$ is a finite set, there is such an $f$ sequence with linear growth of primary decompositions, namely $\left(\left(\mathfrak{a}^{\left[p^{n}\right]}\right)^{F}\right)_{n \in \mathbb{N}_{0}}$.

7.6. Theorem. Assume that $R$ has a $p^{m_{0}}$-weak test element for some $m_{0} \in \mathbb{N}_{0}$; let $\mathfrak{a}$ be a proper ideal of $R$. Suppose that $\left(\mathfrak{a}_{n}\right)_{n \in \mathbb{N}_{0}}$ is an $f$-sequence of ideals of $R$ which has linear growth of primary decompositions and is such that $\mathfrak{a}^{\left[p^{n}\right]} \subseteq \mathfrak{a}_{n} \subseteq\left(\mathfrak{a}^{\left[p^{n}\right]}\right)^{*}$ for all $n \in \mathbb{N}_{0}$.

(i) For each $u \in R$, we have $\mathfrak{a}^{*} R_{u}=\left(\mathfrak{a} R_{u}\right)^{*}$.

(ii) If $\bigcup_{n \in \mathbb{N}_{0}}$ ass $\mathfrak{a}_{n}$ is a finite set, then $\mathfrak{a}^{*} S^{-1} R=\left(\mathfrak{a} S^{-1} R\right)^{*}$ for every multiplicatively closed subset $S$ of $R$.

Note. Note that, if $\left(\mathfrak{a}_{n}^{\prime}\right)_{n \in \mathbb{N}_{0}}$ is an $f$-sequence of ideals of $R$ such that $\mathfrak{a} \subseteq \mathfrak{a}_{0}^{\prime}$, then, for each $n \in \mathbb{N}_{0}$, we have $\mathfrak{a}^{\left[p^{n}\right]} \subseteq \mathfrak{a}_{0}^{\prime\left[p^{n}\right]} \subseteq \mathfrak{a}_{n}^{\prime}$, by $4.2(\mathrm{i})$.

Also, the condition that $\mathfrak{a}^{\left[p^{n}\right]} \subseteq \mathfrak{a}_{n} \subseteq\left(\mathfrak{a}^{\left[p^{n}\right]}\right)^{*}$ (for some $n \in \mathbb{N}_{0}$ ) can be described, in the terminology of [3. (7.11)], by saying that $\mathfrak{a}_{n}$ is trapped over $\mathfrak{a}^{\left[p^{n}\right]}$.

Proof. Let $S$ denote an arbitrary multiplicatively closed subset of $R$. It is clear that $\mathfrak{a}^{*} S^{-1} R \subseteq\left(\mathfrak{a} S^{-1} R\right)^{*}$.

Let $c^{\prime} \in R^{\circ}$ be a $p^{m_{0}}$-weak test element for $R$. Let $r \in R$ be such that $r / 1 \in\left(\mathfrak{a} S^{-1} R\right)^{*}$. Thus there exists $c \in R$ such that $c / 1 \in\left(S^{-1} R\right)^{\circ}$ and $c r^{p^{n}} / 1 \in$ $\left(\mathfrak{a} S^{-1} R\right)^{\left[p^{n}\right]}$ (in the ring of fractions $S^{-1} R$ ) for all $n \gg 0$. If $c \notin R^{\circ}$, let $d$ be an element of $R$ that belongs to those minimal primes of $R$ to which $c$ does not belong, and to no others: an argument in the proof of [3] Proposition 4.14] shows that we may add a suitable power of $d$ to $c$ to see that we can assume that $c \in R^{\circ}$.

By hypothesis, there exists $h \in \mathbb{N}$ such that, for each $n \in \mathbb{N}_{0}$, there is a minimal primary decomposition $\mathfrak{a}_{n}=\mathfrak{q}_{1, n} \cap \cdots \cap \mathfrak{q}_{k_{n}, n}$ with ${\sqrt{\mathfrak{q}_{i, n}}}^{\left[p^{n}\right] h} \subseteq \mathfrak{q}_{i, n}$ for all $i=$ $1, \ldots, k_{n}$.

(i) Here we consider the special case in which $S=\left\{u^{k}: k \in \mathbb{N}_{0}\right\}$, and our conclusion above specialises to the statement that $\mathrm{cr}^{p^{n}} / 1 \in\left(\mathfrak{a} R_{u}\right)^{\left[p^{n}\right]}$ (in the ring of fractions $R_{u}$ ) for all $n \gg 0$, say for all $n \geq n_{0}$. 
Therefore, for each $n \geq n_{0}$, there exists $t(n) \in \mathbb{N}_{0}$ such that $c u^{t(n)} r^{p^{n}} \in \mathfrak{a}^{\left[p^{n}\right]}$. Choose $n \geq n_{0}$ and $i \in\left\{1, \ldots, k_{n}\right\}$. Now $c u^{t(n)} r^{p^{n}} \in \mathfrak{a}^{\left[p^{n}\right]} \subseteq \mathfrak{a}_{n} \subseteq \mathfrak{q}_{i, n}$. If $c r p^{n} \notin \mathfrak{q}_{i, n}$, then $u^{t(n)} \in \sqrt{\mathfrak{q}_{i, n}}$, so that $u \in \sqrt{\mathfrak{q}_{i, n}}$ and $u^{p^{n} h} \in \mathfrak{q}_{i, n}$. It follows that $c\left(r u^{h}\right)^{p^{n}} \in \bigcap_{i=1}^{k_{n}} \mathfrak{q}_{i, n}=\mathfrak{a}_{n} \subseteq\left(\mathfrak{a}^{\left[p^{n}\right]}\right)^{*}$. It should be noted that this is true for each $n \geq n_{0}$. Since $c^{\prime}$ is a $p^{m_{0}}$-weak test element for $R$, we have $c^{\prime}\left(c\left(r u^{h}\right)^{p^{n}}\right)^{p^{m_{0}}} \in$ $\left(\mathfrak{a}^{\left[p^{n}\right]}\right)^{\left[p^{m_{0}}\right]}$ for all $n \geq n_{0}$. Therefore $c^{\prime} c^{p^{m_{0}}}\left(r u^{h}\right)^{p^{n+m_{0}}} \in \mathfrak{a}^{\left[p^{n+m_{0}}\right]}$ for all $n \geq n_{0}$. Since $c^{\prime} c^{p^{m_{0}}} \in R^{\circ}$, it follows that $r u^{h} \in \mathfrak{a}^{*}$, so that $r / 1=r u^{h} / u^{h} \in \mathfrak{a}^{*} R_{u}$.

(ii) Here, we revert to the situation where $S$ is an arbitrary multiplicatively closed subset of $R$.

Note that, by 4.2 (iv), ass $\mathfrak{a}_{n} \subseteq$ ass $\mathfrak{a}_{n+1}$ for all $n \in \mathbb{N}_{0}$. Suppose that the finite set $\bigcup_{n \in \mathbb{N}_{0}}$ ass $\mathfrak{a}_{n}$ has $t$ elements $\mathfrak{p}_{1}, \ldots, \mathfrak{p}_{t}$. We can therefore relabel the terms in the above-mentioned minimal primary decompositions so that $\sqrt{\mathfrak{q}_{i, n}}=\mathfrak{p}_{i}$ for all $i=1, \ldots, k_{n}$ and all $n \in \mathbb{N}_{0}$. It is notationally convenient to define, for any $n \in \mathbb{N}_{0}$ for which $k_{n}<t$ and any $j \in\left\{k_{n}+1, \ldots, t\right\}$, an additional $\mathfrak{p}_{j}$-primary ideal $\mathfrak{q}_{j, n}$ by $\mathfrak{q}_{j, n}=f^{-\left(n^{\prime}-n\right)}\left(\mathfrak{q}_{j, n^{\prime}}\right)$, where $n^{\prime}$ is chosen so large that $j \leq k_{n^{\prime}}$. We shall then have, for every $n \in \mathbb{N}_{0}$, a (not necessarily minimal) primary decomposition $\mathfrak{a}_{n}=\mathfrak{q}_{1, n} \cap \cdots \cap \mathfrak{q}_{t, n}$ with $\sqrt{\mathfrak{q}_{i, n}}\left[p^{n}\right] h=\mathfrak{p}_{i}^{\left[p^{n}\right] h} \subseteq \mathfrak{q}_{i, n}$ for all $i=1, \ldots, t$. (The extra primary ideals will also have the necessary properties to ensure that this holds.)

Recall that we have found $c \in R^{\circ}$ such that $c r^{p^{n}} / 1 \in\left(\mathfrak{a} S^{-1} R\right)^{\left[p^{n}\right]}$ (in the ring of fractions $S^{-1} R$ ) for all $n \gg 0$, say for all $n \geq n_{0}$. Hence, for each $n \geq n_{0}$, there exists $s_{n} \in S$ such that $c s_{n} r^{p^{n}} \in \mathfrak{a}^{\left[p^{n}\right]} \subseteq \mathfrak{a}_{n}=\mathfrak{q}_{1, n} \cap \cdots \cap \mathfrak{q}_{t, n}$. Choose $j \in\{1, \ldots, t\}$. Suppose that there exists an $m \geq n_{0}$ such that $\mathrm{cr}^{p^{m}} \notin \mathfrak{q}_{j, m}$, and choose the least such $m$. Then $s_{m} \in \sqrt{\mathfrak{q}_{j, m}}=\mathfrak{p}_{j}$ : define $s_{\mathfrak{p}_{j}}$ to be this $s_{m}$, and note that $s_{\mathfrak{p}_{j}}^{h p^{n}} \in \mathfrak{q}_{j, n}$ for all $n \in \mathbb{N}_{0}$. If, on the other hand, $c r^{p^{n}} \in \mathfrak{q}_{j, n}$ for all $n \geq n_{0}$, set $s_{\mathfrak{p}_{j}}=1$. In both cases, we have $c s_{\mathfrak{p}_{j}}^{h p^{n}} r^{p^{n}} \in \mathfrak{q}_{j, n}$ for all $n \geq n_{0}$.

Set $s:=s_{\mathfrak{p}_{1}} \ldots s_{\mathfrak{p}_{t}} \in S$; we have $c\left(r s^{h}\right)^{p^{n}} \in \bigcap_{j=1}^{t} \mathfrak{q}_{j, n}=\mathfrak{a}_{n} \subseteq\left(\mathfrak{a}^{\left[p^{n}\right]}\right)^{*}$ for all $n \geq$ $n_{0}$. Since $c^{\prime}$ is a $p^{m_{0}}$-weak test element for $R$, we have $c^{\prime}\left(c\left(r s^{h}\right)^{p^{n}}\right)^{p^{m_{0}}} \in\left(\mathfrak{a}^{\left[p^{n}\right]}\right)^{\left[p^{m_{0}}\right]}$ for all $n \geq n_{0}$; thus $c^{\prime} c^{p^{m_{0}}}\left(r s^{h}\right)^{p^{n+m_{0}}} \in \mathfrak{a}^{\left[p^{n+m_{0}}\right]}$ for all $n \geq n_{0}$. Since $c^{\prime} c^{p^{m_{0}}} \in R^{\circ}$, it follows that $r s^{h} \in \mathfrak{a}^{*}$, so that $r / 1=r s^{h} / s^{h} \in \mathfrak{a}^{*} S^{-1} R$.

Our next major aim is to establish that, if a proper ideal $\mathfrak{A}$ of $R^{\infty}$ has a primary decomposition, then the $f$-sequence $\left(\mathfrak{a}_{n}\right)_{n \in \mathbb{N}_{0}}$ of ideals of $R$ to which it corresponds has linear growth of primary decompositions, and $\bigcup_{n \in \mathbb{N}_{0}}$ ass $\mathfrak{a}_{n}$ is finite. This will enable us to exploit Theorem 7.6(ii). We need one preparatory lemma.

7.7. Lemma. Let $\mathfrak{p} \in \operatorname{Spec}(R)$ and let $\left(\mathfrak{q}_{n}\right)_{n \in \mathbb{N}_{0}}$ be an $f$-sequence of $\mathfrak{p}$-primary ideals of $R$. Let $h \in \mathbb{N}$ be such that $\mathfrak{p}^{h} \subseteq \mathfrak{q}_{0}$. Then $\mathfrak{p}^{\left[p^{n}\right] h}={\sqrt{\mathfrak{q}_{n}}}^{\left[p^{n}\right] h} \subseteq \mathfrak{q}_{n}$ for all $n \in \mathbb{N}_{0}$ (so that, in the language of 7.1 the $f$-sequence $\left(\mathfrak{q}_{n}\right)_{n \in \mathbb{N}_{0}}$ has h-linear growth of primary decompositions).

Proof. By $4.2(\mathrm{i})$, we have $\mathfrak{q}_{0}^{\left[p^{n}\right]} \subseteq \mathfrak{q}_{n} \subseteq \mathfrak{q}_{0}$ for all $n \in \mathbb{N}_{0}$. As $R$ is Noetherian, there exists $h \in \mathbb{N}$ such that $\mathfrak{p}^{h} \subseteq \mathfrak{q}_{0}$. Then $\mathfrak{q}_{n} \supseteq \mathfrak{q}_{0}^{\left[p^{n}\right]} \supseteq\left(\mathfrak{p}^{h}\right)^{\left[p^{n}\right]}={\sqrt{\mathfrak{q}_{0}}}^{\left[p^{n}\right] h}={\sqrt{\mathfrak{q}_{n}}}^{\left[p^{n}\right] h}$ for all $n \in \mathbb{N}_{0}$.

7.8. Remark. Let $\mathfrak{p} \in \operatorname{Spec}(R)$ and let $\left(\mathfrak{q}_{n}\right)_{n \in \mathbb{N}_{0}}$ be an $f$-sequence of $\mathfrak{p}$-primary ideals of $R$. Let $n \in \mathbb{N}_{0}$. By $4.2(\mathrm{i})$, we have $\left(\mathfrak{q}_{0}^{\left[p^{n}\right]}\right)^{F} \subseteq \mathfrak{q}_{n}$. Let ${ }^{e}$ and ${ }^{c}$ stand for extension and contraction with respect to the natural ring homomorphism $R \longrightarrow$ $R_{\mathfrak{p}}$. Then $\left(\left(\mathfrak{q}_{0}^{\left[p^{n}\right]}\right)^{F}\right)^{e c} \subseteq \mathfrak{q}_{n}$, since $\mathfrak{q}_{n}$ is $\mathfrak{p}$-primary. A simple example quickly shows 
that we cannot expect equality here: let $K$ be a field of characteristic $p$, take $R=K[X]$, the polynomial ring in one indeterminate, and take $\mathfrak{q}_{n}=(X)$ for all $n \in \mathbb{N}_{0}$.

7.9. Theorem. If the proper ideal $\mathfrak{A}$ of $R^{\infty}$ has a primary decomposition, then the $f$-sequence $\left(\mathfrak{a}_{n}\right)_{n \in \mathbb{N}_{0}}$ of ideals of $R$ corresponding to $\mathfrak{A}$ has linear growth of primary decompositions, and $\bigcup_{n \in \mathbb{N}_{0}}$ ass $\mathfrak{a}_{n}$ is a finite set.

Proof. By Theorem 6.4, there are prime ideals $\mathfrak{p}_{1}, \ldots, \mathfrak{p}_{t}$ of $R$ such that the following is true: each $\mathfrak{a}_{n}$ has a primary decomposition $\mathfrak{a}_{n}=\mathfrak{q}_{1, n} \cap \cdots \cap \mathfrak{q}_{t, n}$ such that $\mathfrak{q}_{k, n}$ is $\mathfrak{p}_{k}$-primary for all $k=1, \ldots, t$ and all $n \in \mathbb{N}_{0}$, and $\left(\mathfrak{q}_{k, n}\right)_{n \in \mathbb{N}_{0}}$ is an $f$-sequence for all $k=1, \ldots, t$.

Now there exist positive integers $h_{1}, \ldots, h_{t}$ such that $\sqrt{\mathfrak{q}_{k, 0}} h_{k} \subseteq \mathfrak{q}_{k, 0}$ for all $k=1, \ldots, t$. By Lemma [7.7] we have ${\sqrt{\mathfrak{q}_{k, n}}}^{\left[p^{n}\right] h_{k}} \subseteq \mathfrak{q}_{k, n}$ for all $n \in \mathbb{N}_{0}$ and all $k=1, \ldots, t$. Hence, with $h:=\max \left\{h_{1}, \ldots, h_{t}\right\}$, we have ${\sqrt{\mathfrak{q}_{k, n}}}^{\left[p^{n}\right] h} \subseteq \mathfrak{q}_{k, n}$ for all $n \in \mathbb{N}_{0}$ and all $k=1, \ldots, t$. The final claim also follows from Theorem 6.4.

In view of Theorems 7.6 (ii) and 7.9 we are very interested in finding primary decompositions of proper ideals of $R^{\infty}$. However, in Theorem 6.3 we showed that $R^{\infty}$ is only Noetherian in rather uninteresting cases, and so the existence of a primary decomposition for a proper ideal of $R^{\infty}$ would be a bonus that we should not expect in all cases. Indeed, Example 7.10 below is of an $f$-sequence $\left(\mathfrak{a}_{n}\right)_{n \in \mathbb{N}_{0}}$ of proper ideals (in a 2-dimensional regular ring $R$ ) which has linear growth of primary decompositions, but for which the set $\bigcup_{n \in \mathbb{N}_{0}}$ ass $\mathfrak{a}_{n}$ is infinite (so that, by Theorem 7.9, the associated ideal of $R^{\infty}$ cannot have a primary decomposition).

7.10. Example. Let $K$ be an infinite field of prime characteristic $p$, let $\left(\lambda_{j}\right)_{j \in \mathbb{N}}$ be a sequence of distinct elements of $K$, and let $\left(t_{j}\right)_{j \in \mathbb{N}}$ be a sequence of positive integers. Let $R=K[X, Y]$, where $X$ and $Y$ are independent indeterminates. Let $l$ be an integer such that $2 \leq l \leq p$.

Let $\left(\mathfrak{q}_{0, n}\right)_{n \in \mathbb{N}_{0}}$ be the $f$-sequence of ideals of $R$ for which $\mathfrak{q}_{0, n}=(X)$ for all $n \in \mathbb{N}_{0}$; see 6.1)(i).

Note that $R$ is regular, so that, if $\mathfrak{a}$ is an arbitrary ideal of $R$, then $\left(\mathfrak{a}^{\left[p^{n}\right]}\right)_{n \in \mathbb{N}_{0}}$ is the $f$-sequence $\left(\left(\mathfrak{a}^{\left[p^{n}\right]}\right)^{F}\right)_{n \in \mathbb{N}_{0}}$, since every ideal of $R$ is tightly closed (see 4.1(iii)). With this and 4.2 (iii) in mind, we let, for each $j \in \mathbb{N},\left(\mathfrak{q}_{j, n}\right)_{n \in \mathbb{N}_{0}}$ be the $f$-sequence of $\left(X,\left(Y-\lambda_{j}\right)\right)$-primary ideals of $R$ given by

$$
\mathfrak{q}_{j, n}= \begin{cases}\left(X^{l p^{n-j}},\left(Y-\lambda_{j}\right)^{t_{j} p^{n}}\right)=\left(X^{l},\left(Y-\lambda_{j}\right)^{t_{j} p^{j}}\right)^{\left[p^{n-j}\right]} & \text { if } n \geq j \\ f^{-(j-n)}\left(\mathfrak{q}_{j, j}\right) & \text { if } n<j\end{cases}
$$

Let $\left(\mathfrak{a}_{n}\right)_{n \in \mathbb{N}_{0}}$ be the $f$-sequence of ideals of $R$ given by $\mathfrak{a}_{n}:=\bigcap_{j \in \mathbb{N}_{0}} \mathfrak{q}_{j, n}$ for all $n \in$ $\mathbb{N}_{0}$; see6.1(v). Note that, for $m, j \in \mathbb{N}_{0}$ with $j>m$, we have $f^{j-m}(X)=X^{p^{j-m}}=$ $X^{l} X^{p^{j-m}-l} \in\left(X^{l},\left(Y-\lambda_{j}\right)^{t_{j} p^{j}}\right)=\mathfrak{q}_{j, j}$; hence $\mathfrak{q}_{0, m}=(X) \subseteq f^{-(j-m)}\left(\mathfrak{q}_{j, j}\right)=\mathfrak{q}_{j, m}$ for all $j>m$. Thus

$\mathfrak{a}_{m}=(X) \cap\left(X^{l p^{m-1}},\left(Y-\lambda_{1}\right)^{t_{1} p^{m}}\right) \cap\left(X^{l p^{m-2}},\left(Y-\lambda_{2}\right)^{t_{2} p^{m}}\right) \cap \cdots \cap\left(X^{l},\left(Y-\lambda_{m}\right)^{t_{m} p^{m}}\right)$.

It therefore follows that $\mathfrak{a}_{m}=\mathfrak{q}_{0, m} \cap \mathfrak{q}_{1, m} \cap \cdots \cap \mathfrak{q}_{m, m}$ is a primary decomposition (in which the radicals of the primary terms are all different), for each $m \in \mathbb{N}_{0}$. We show next, by induction on $m$, that these primary decompositions are all minimal; it is 
clear that $\mathfrak{a}_{0}=\mathfrak{q}_{0,0}$ is a minimal primary decomposition, and so we now suppose that $m>0$ and make the inductive hypothesis that

$$
\mathfrak{a}_{m-1}=\mathfrak{q}_{0, m-1} \cap \mathfrak{q}_{1, m-1} \cap \cdots \cap \mathfrak{q}_{m-1, m-1}
$$

is a minimal primary decomposition.

Since $\left(\mathfrak{a}_{n}\right)_{n \in \mathbb{N}_{0}}$ is an $f$-sequence, ass $\mathfrak{a}_{m-1} \subseteq$ ass $\mathfrak{a}_{m}$ by 4.2(iv). Therefore, none of $\mathfrak{q}_{0, m}, \ldots, \mathfrak{q}_{m-1, m}$ can be omitted from the primary decomposition $\mathfrak{a}_{m}=\bigcap_{k=0}^{m} \mathfrak{q}_{k, m}$. We can then conclude that this primary decomposition is minimal simply by observing that

$$
X\left(Y-\lambda_{1}\right)^{t_{1} p^{m}} \cdots\left(Y-\lambda_{m-1}\right)^{t_{m-1} p^{m}} \in\left(\mathfrak{q}_{0, m} \cap \mathfrak{q}_{1, m} \cap \cdots \cap \mathfrak{q}_{m-1, m}\right) \backslash \mathfrak{q}_{m, m} .
$$

A consequence of this inductive argument is that $\left(\mathfrak{a}_{n}\right)_{n \in \mathbb{N}_{0}}$ is an $f$-sequence of ideals of $R$ for which

$$
\operatorname{ass} \mathfrak{a}_{n}=\left\{(X),\left(X,\left(Y-\lambda_{1}\right)\right), \ldots,\left(X,\left(Y-\lambda_{n}\right)\right)\right\} \quad \text { for all } n \in \mathbb{N}_{0} .
$$

(Of course, in the case when $n=0$, this statement is to be interpreted as 'ass $\mathfrak{a}_{0}=$ $\left.\{(X)\}^{\prime}.\right)$ It therefore follows from Theorem 6.4 that the ideal of $R^{\infty}$ to which $\left(\mathfrak{a}_{n}\right)_{n \in \mathbb{N}_{0}}$ corresponds does not have a primary decomposition. However, if the sequence $\left(t_{j}\right)_{j \in \mathbb{N}}$ is chosen so that it is bounded, by an integer $t$ say, then it is straightforward to check that ${\sqrt{\mathfrak{q}_{j, n}}}^{\left[p^{n}\right] t} \subseteq \mathfrak{q}_{j, n}$ for all $n, j \in \mathbb{N}_{0}$ with $j \leq n$; thus $\left(\mathfrak{a}_{n}\right)_{n \in \mathbb{N}_{0}}$ does have linear growth of primary decompositions.

We revert now to the general situation of our standard hypotheses. Our next result will show that the primary components of the terms $\mathfrak{a}_{n}$ in an $f$-sequence $\left(\mathfrak{a}_{n}\right)_{n \in \mathbb{N}_{0}}$ corresponding to the minimal prime ideals of the $f$-sequence (see $4.2(\mathrm{v})$ ) never present any obstacle to the $f$-sequence's having linear growth of primary decompositions.

7.11. Proposition. Let $\left(\mathfrak{a}_{n}\right)_{n \in \mathbb{N}_{0}}$ be an $f$-sequence of proper ideals of $R$, and let $\mathfrak{p}_{1}, \ldots, \mathfrak{p}_{l}$ be the minimal primes of this $f$-sequence (see $4.2(\mathrm{v})$ ). For each $n \in \mathbb{N}_{0}$ and each $k \in\{1, \ldots, l\}$, let $\mathfrak{q}_{k, n}$ be the (uniquely determined) $\mathfrak{p}_{k}$-primary component of $\mathfrak{a}_{n}$. Let $k_{1}, \ldots, k_{t}$ be integers with $1 \leq k_{1}<k_{2}<\cdots<k_{t} \leq l$. Then $\left(\mathfrak{q}_{k_{1}, n} \cap \mathfrak{q}_{k_{2}, n} \cap \cdots \cap \mathfrak{q}_{k_{t}, n}\right)_{n \in \mathbb{N}_{0}}$ is an $f$-sequence of ideals of $R$ which has linear growth of primary decompositions, and the ideal of $R^{\infty}$ to which it corresponds has a primary decomposition.

Proof. Set $S:=R \backslash \bigcup_{j=1}^{t} \mathfrak{p}_{k_{j}}$, a multiplicatively closed subset of $R$. Let $e$ and ${ }^{c}$ stand for extension and contraction with respect to the natural ring homomorphism $R \longrightarrow S^{-1} R$. By 5.5, the sequence $\left(\mathfrak{a}_{n}^{e c}\right)_{n \in \mathbb{N}_{0}}$ is an $f$-sequence of ideals of $R$. But $\mathfrak{a}_{n}^{e c}=\mathfrak{q}_{k_{1}, n} \cap \mathfrak{q}_{k_{2}, n} \cap \cdots \cap \mathfrak{q}_{k_{t}, n}$ for all $n \in \mathbb{N}_{0}$. As particular cases, we see that, for each $j=1, \ldots, t$, the sequence $\left(\mathfrak{q}_{k_{j}, n}\right)_{n \in \mathbb{N}_{0}}$ is an $f$-sequence. It therefore follows from Theorem 6.4 that the ideal of $R^{\infty}$ to which the $f$-sequence $\left(\mathfrak{a}_{n}^{e c}\right)_{n \in \mathbb{N}_{0}}$ corresponds has a primary decomposition. Hence $\left(\mathfrak{a}_{n}^{e c}\right)_{n \in \mathbb{N}_{0}}$ has linear growth of primary decompositions, by Theorem 7.9

7.12. Corollary. Let $\left(\mathfrak{a}_{n}\right)_{n \in \mathbb{N}_{0}}$ be an $f$-sequence of proper ideals of $R$ with the property that each $\mathfrak{a}_{n}\left(n \in \mathbb{N}_{0}\right)$ has no embedded prime. Then $\left(\mathfrak{a}_{n}\right)_{n \in \mathbb{N}_{0}}$ has linear growth of primary decompositions, and the corresponding ideal of $R^{\infty}$ has a primary decomposition. 
Thus the problems, in showing that a given $f$-sequence $\left(\mathfrak{a}_{n}\right)_{n \in \mathbb{N}_{0}}$ of proper ideals of $R$ has linear growth of primary decompositions, rest entirely with the embedded primary components of the $\mathfrak{a}_{n}$. None of these is ever uniquely determined, and the issues revolve around whether or not it is possible to make appropriate choices for these embedded primary components. In (the proof of) Theorem 7.9 above, we saw that, if the ideal of $R^{\infty}$ corresponding to the $f$-sequence $\left(\mathfrak{a}_{n}\right)_{n \in \mathbb{N}_{0}}$ has a primary decomposition, then there are natural choices for the above-mentioned embedded primary components that satisfy the conditions necessary for linear growth of primary decompositions.

In the next section, we shall present some examples of proper ideals in nonNoetherian perfect closures that do have primary decompositions.

The results of this section provide a strategy for attempting to show that, for a given proper ideal $\mathfrak{a}$ of $R$, tight closure commutes with localization with respect to an arbitrary multiplicatively closed subset of $R$ : if we can find an $f$-sequence $\left(\mathfrak{a}_{n}\right)_{n \in \mathbb{N}_{0}}$ of ideals of $R$ such that $\mathfrak{a}_{n}$ is trapped over $\mathfrak{a}^{\left[p^{n}\right]}$ for all $n \in \mathbb{N}_{0}$, if $R$ has a $p^{m_{0}}$-weak test element (for some $m_{0} \in \mathbb{N}_{0}$ ) and if the ideal of $R^{\infty}$ to which $\left(\mathfrak{a}_{n}\right)_{n \in \mathbb{N}_{0}}$ corresponds has a primary decomposition, then it follows from Theorem [7.9 that $\left(\mathfrak{a}_{n}\right)_{n \in \mathbb{N}_{0}}$ has linear growth of primary decompositions and that $\bigcup_{n \in \mathbb{N}_{0}}$ ass $\mathfrak{a}_{n}$ is a finite set, and it then follows from Theorem 7.6(ii) that $\mathfrak{a}^{*} S^{-1} R=\left(\mathfrak{a} S^{-1} R\right)^{*}$ for every multiplicatively closed subset $S$ of $R$. We should perhaps mention at this point that, by-and-large, we have only managed to get this strategy to succeed in situations where it had already been proved that tight closure commutes with localization. However, our next result shows that the above-mentioned hypotheses needed for the strategy to work actually ensure that the $f$-sequence $\left(\left(\mathfrak{a}^{\left[p^{n}\right]}\right)^{*}\right)_{n \in \mathbb{N}_{0}}$ of tight closures of the Frobenius powers of $\mathfrak{a}$ (see Lemma 5.1) also has linear growth of primary decompositions, and that $\bigcup_{n \in \mathbb{N}_{0}} \operatorname{ass}\left(\mathfrak{a}^{\left[p^{n}\right]}\right)^{*}$ is finite.

This is interesting, because M. Katzman's approach in 10] to the localization problem for tight closure led to the following question: is it the case that, for every ideal $\mathfrak{b}$ of $R$, the set $\bigcup_{n \in \mathbb{N}_{0}} \operatorname{ass}\left(\mathfrak{b}^{\left[p^{n}\right]}\right)^{*}$ has only finitely many maximal elements? Our strategy outlined above, used in conjunction with Theorem 7.13 below, will enable us to conclude in $₫ 8$ that $\bigcup_{n \in \mathbb{N}_{0}}$ ass $\left(\mathfrak{b}^{\left[p^{n}\right]}\right)^{*}$ is actually a finite set in several cases where it is known that, for $\mathfrak{b}$, tight closure commutes with localization with respect to an arbitrary multiplicatively closed subset of $R$.

7.13. Theorem. Let $\mathfrak{a}$ be a proper ideal of $R$. Suppose that $\left(\mathfrak{a}_{n}\right)_{n \in \mathbb{N}_{0}}$ is an $f$ sequence of ideals of $R$ which is such that $\mathfrak{a}^{\left[p^{n}\right]} \subseteq \mathfrak{a}_{n} \subseteq\left(\mathfrak{a}^{\left[p^{n}\right]}\right)^{*}$ for all $n \in \mathbb{N}_{0}$, and that the ideal $\mathfrak{A}$ of $R^{\infty}$ to which $\left(\mathfrak{a}_{n}\right)_{n \in \mathbb{N}_{0}}$ corresponds has a primary decomposition, so that, by 7.9, the set $\bigcup_{n \in \mathbb{N}_{0}}$ ass $\mathfrak{a}_{n}$ is finite. Let the members of the latter set be $\mathfrak{p}_{1}, \ldots, \mathfrak{p}_{t}$, and let the corresponding prime ideals of $R^{\infty}$ (see 6.1 (i)) be $\mathfrak{P}_{1}, \ldots, \mathfrak{P}_{t}$, respectively.

For each $i=1, \ldots, t$, let $e_{i}$ and $c_{i}$ stand for extension and contraction with respect to the natural ring homomorphism $R \longrightarrow R_{\mathfrak{p}_{i}}$.

(i) There exists $h \in \mathbb{N}$ such that, for each $n \in \mathbb{N}_{0}$,

$$
\mathfrak{a}_{n}=\bigcap_{i=1}^{t}\left(\left(\mathfrak{a}_{n}+\mathfrak{p}_{i}^{h\left[p^{n}\right]}\right)^{F}\right)^{e_{i} c_{i}}
$$

is a primary decomposition in which each primary component is F-closed, where $\left(\left(\mathfrak{a}_{n}+\mathfrak{p}_{i}^{h\left[p^{n}\right]}\right)^{F}\right)^{e_{i} c_{i}}$ is $\mathfrak{p}_{i}$-primary for $i=1, \ldots, t$. 
(ii) Assume that $R$ has a $p^{m_{0}}$-weak test element, for some $m_{0} \in \mathbb{N}_{0}$. Then, with $h$ as in (i),

(a) for each $n \in \mathbb{N}_{0}$,

$$
\left(\mathfrak{a}^{\left[p^{n}\right]}\right)^{*}=\bigcap_{i=1}^{t}\left(\left(\left(\mathfrak{a}+\mathfrak{p}_{i}^{h}\right)^{\left[p^{n}\right]}\right)^{*}\right)^{e_{i} c_{i}}
$$

is a primary decomposition in which each primary component is $F$ closed, where $\left(\left(\left(\mathfrak{a}+\mathfrak{p}_{i}^{h}\right)^{\left[p^{n}\right]}\right)^{*}\right)^{e_{i} c_{i}}$ is $\mathfrak{p}_{i}$-primary for $i=1, \ldots, t$, and the ideal of $R^{\infty}$ to which the $f$-sequence $\left(\left(\mathfrak{a}^{\left[p^{n}\right]}\right)^{*}\right)_{n \in \mathbb{N}_{0}}$ corresponds has a primary decomposition;

(b) the $f$-sequence $\left(\left(\mathfrak{a}^{\left[p^{n}\right]}\right)^{*}\right)_{n \in \mathbb{N}_{0}}$ has h-linear growth of primary decompositions, and the set $\bigcup_{n \in \mathbb{N}_{0}} \operatorname{ass}\left(\mathfrak{a}^{\left[p^{n}\right]}\right)^{*}$ is finite; and

(c) $\bigcup_{n \in \mathbb{N}_{0}} \operatorname{ass}\left(\mathfrak{a}^{\left[p^{n}\right]}\right)^{*} \subseteq \bigcup_{n \in \mathbb{N}_{0}}$ ass $\mathfrak{a}_{n}$.

(iii) If $R$ has a locally stable $p^{m_{0}}$-weak test element, for some $m_{0} \in \mathbb{N}_{0}$, then, for each $n \in \mathbb{N}_{0}$, and with $h$ as in (i),

$$
\left(\mathfrak{a}^{\left[p^{n}\right]}\right)^{*}=\bigcap_{i=1}^{t}\left(\left(\left(\left(\mathfrak{a}+\mathfrak{p}_{i}^{h}\right)^{\left[p^{n}\right]}\right)^{e_{i}}\right)^{*}\right)^{c_{i}}
$$

is a primary decomposition in which each primary component is tightly closed, where $\left(\left(\left(\left(\mathfrak{a}+\mathfrak{p}_{i}^{h}\right)^{\left[p^{n}\right]}\right)^{e_{i}}\right)^{*}\right)^{c_{i}}$ is $\mathfrak{p}_{i}$-primary for $i=1, \ldots, t$.

Proof. By Theorems 6.4 and 7.9 each $\mathfrak{a}_{n}$ has a primary decomposition

$$
\mathfrak{a}_{n}=\mathfrak{q}_{1, n} \cap \cdots \cap \mathfrak{q}_{t, n}
$$

such that $\left(\mathfrak{q}_{i, n}\right)_{n \in \mathbb{N}_{0}}$ is an $f$-sequence of $\mathfrak{p}_{i}$-primary ideals of $R$ for all $i=1, \ldots, t$, and there exists $h \in \mathbb{N}$ such that $\sqrt{\mathfrak{q}_{i, n}} h\left[p^{n}\right]=\mathfrak{p}_{i}^{h\left[p^{n}\right]} \subseteq \mathfrak{q}_{i, n}=\mathfrak{q}_{i, n}^{F}$ for all $n \in \mathbb{N}_{0}$ and all $i=1, \ldots, t$.

(i) Now $\mathfrak{a}_{n} \subseteq \mathfrak{a}_{n}+\mathfrak{p}_{i}^{h\left[p^{n}\right]} \subseteq \mathfrak{q}_{i, n}$, so that

$$
\mathfrak{a}_{n} \subseteq\left(\left(\mathfrak{a}_{n}+\mathfrak{p}_{i}^{h\left[p^{n}\right]}\right)^{F}\right)^{e_{i} c_{i}} \subseteq\left(\mathfrak{q}_{i, n}^{F}\right)^{e_{i} c_{i}}=\mathfrak{q}_{i, n}^{e_{i} c_{i}}=\mathfrak{q}_{i, n} \quad \text { for all } i=1, \ldots, t .
$$

Hence $\mathfrak{a}_{n}=\bigcap_{i=1}^{t}\left(\left(\mathfrak{a}_{n}+\mathfrak{p}_{i}^{h\left[p^{n}\right]}\right)^{F}\right)^{e_{i} c_{i}} ;$ moreover

$$
\mathfrak{p}_{i}^{h\left[p^{n}\right]} \subseteq\left(\mathfrak{a}_{n}+\mathfrak{p}_{i}^{h\left[p^{n}\right]}\right)^{e_{i} c_{i}} \subseteq\left(\left(\mathfrak{a}_{n}+\mathfrak{p}_{i}^{h\left[p^{n}\right]}\right)^{F}\right)^{e_{i} c_{i}} \subseteq \mathfrak{p}_{i} \quad \text { for all } i=1, \ldots, t,
$$

so that $\left(\left(\mathfrak{a}_{n}+\mathfrak{p}_{i}^{h\left[p^{n}\right]}\right)^{F}\right)^{e_{i} c_{i}}$ is $\mathfrak{p}_{i}$-primary. It follows that

$$
\mathfrak{a}_{n}=\bigcap_{i=1}^{t}\left(\left(\mathfrak{a}_{n}+\mathfrak{p}_{i}^{h\left[p^{n}\right]}\right)^{F}\right)^{e_{i} c_{i}}
$$

is a primary decomposition, where $\left(\left(\mathfrak{a}_{n}+\mathfrak{p}_{i}^{h\left[p^{n}\right]}\right)^{F}\right)^{e_{i} c_{i}}$ is $\mathfrak{p}_{i}$-primary for $i=1, \ldots, t$, and parts (iii) and (i) of Lemma 7.4 show that each primary component in this decomposition is $F$-closed.

(ii) Let $c$ be a $p^{m_{0}}$-weak test element for $R$.

It is clear that $\left(\mathfrak{a}^{\left[p^{n}\right]}\right)^{*} \subseteq \bigcap_{i=1}^{t}\left(\left(\mathfrak{a}+\mathfrak{p}_{i}^{h}\right)^{\left[p^{n}\right]}\right)^{*} \subseteq \bigcap_{i=1}^{t}\left(\left(\left(\mathfrak{a}+\mathfrak{p}_{i}^{h}\right)^{\left[p^{n}\right]}\right)^{*}\right)^{e_{i} c_{i}}$. Note that, for each $i \in\{1, \ldots, t\}$, we have $\mathfrak{p}_{i}^{\left[p^{n}\right] h} \subseteq\left(\mathfrak{a}+\mathfrak{p}_{i}^{h}\right)^{\left[p^{n}\right]} \subseteq \mathfrak{p}_{i}$, so that

$$
\mathfrak{p}_{i}^{\left[p^{n}\right] h} \subseteq\left(\left(\mathfrak{a}+\mathfrak{p}_{i}^{h}\right)^{\left[p^{n}\right]}\right)^{*} \subseteq\left(\left(\left(\mathfrak{a}+\mathfrak{p}_{i}^{h}\right)^{\left[p^{n}\right]}\right)^{*}\right)^{e_{i} c_{i}} \subseteq\left(\mathfrak{p}_{i}^{*}\right)^{e_{i} c_{i}}=\mathfrak{p}_{i}^{e_{i} c_{i}}=\mathfrak{p}_{i} .
$$


It therefore follows that $\left(\left(\left(\mathfrak{a}+\mathfrak{p}_{i}^{h}\right)^{\left[p^{n}\right]}\right)^{*}\right)^{e_{i} c_{i}}$ is $\mathfrak{p}_{i}$-primary. We show next that $\bigcap_{i=1}^{t}\left(\left(\left(\mathfrak{a}+\mathfrak{p}_{i}^{h}\right)^{\left[p^{n}\right]}\right)^{*}\right)^{e_{i} c_{i}} \subseteq\left(\mathfrak{a}^{\left[p^{n}\right]}\right)^{*}$.

So let $r \in \bigcap_{i=1}^{t}\left(\left(\left(\mathfrak{a}+\mathfrak{p}_{i}^{h}\right)^{\left[p^{n}\right]}\right)^{*}\right)^{e_{i} c_{i}}$. Then, for each $i \in\{1, \ldots, t\}$, there exists $s_{i} \in R \backslash \mathfrak{p}_{i}$ such that $s_{i} r \in\left(\left(\mathfrak{a}+\mathfrak{p}_{i}^{h}\right)^{\left[p^{n}\right]}\right)^{*}$; therefore, for all $m \geq m_{0}$, we have $c\left(s_{i} r\right)^{p^{m}} \in\left(\left(\mathfrak{a}+\mathfrak{p}_{i}^{h}\right)^{\left[p^{n}\right]}\right)^{\left[p^{m}\right]}$. Therefore

$$
\begin{aligned}
c s_{i}^{p^{m}} r^{p^{m}} & \in \mathfrak{a}^{\left[p^{n+m}\right]}+\mathfrak{p}_{i}^{h\left[p^{n+m}\right]} \subseteq \mathfrak{a}_{n+m}+\mathfrak{p}_{i}^{h\left[p^{n+m}\right]} \\
& \subseteq\left(\mathfrak{a}_{n+m}+\mathfrak{p}_{i}^{h\left[p^{n+m}\right]}\right)^{F} \quad \text { for all } m \geq m_{0} .
\end{aligned}
$$

Hence, for all $i=1, \ldots, t$, we have $c r^{p^{m}} \in\left(\left(\mathfrak{a}_{n+m}+\mathfrak{p}_{i}^{h\left[p^{n+m}\right]}\right)^{F}\right)^{e_{i} c_{i}}$ for all $m \geq m_{0}$. One can now use part (i) to deduce that $c r^{p^{m}} \in \mathfrak{a}_{n+m} \subseteq\left(\mathfrak{a}^{\left[p^{n+m}\right]}\right)^{*}$ for all $m \geq m_{0}$.

We again use the fact that $c$ is a $p^{m_{0}}$-weak test element to deduce that

$$
c\left(c r^{p^{m}}\right)^{p^{k}} \in\left(\mathfrak{a}^{\left[p^{n+m}\right]}\right)^{\left[p^{k}\right]}=\mathfrak{a}^{\left[p^{n+m+k}\right]} \quad \text { for all } m, k \geq m_{0} .
$$

Take $k=m_{0}$ to see that $c^{1+p^{m_{0}}} r^{p^{m+m_{0}}} \in\left(\mathfrak{a}^{\left[p^{n}\right]}\right)^{\left[p^{m+m_{0}}\right]}$ for all $m \geq m_{0}$. Since $c^{1+p^{m_{0}}} \in R^{\circ}$, it follows that $r \in\left(\mathfrak{a}^{\left[p^{n}\right]}\right)^{*}$. Therefore

$$
\left(\mathfrak{a}^{\left[p^{n}\right]}\right)^{*}=\bigcap_{i=1}^{t}\left(\left(\left(\mathfrak{a}+\mathfrak{p}_{i}^{h}\right)^{\left[p^{n}\right]}\right)^{*}\right)^{e_{i} c_{i}}
$$

is a primary decomposition, where $\left(\left(\left(\mathfrak{a}+\mathfrak{p}_{i}^{h}\right)^{\left[p^{n}\right]}\right)^{*}\right)^{e_{i} c_{i}}$ is $\mathfrak{p}_{i}$-primary for $i=1, \ldots, t$. Since a tightly-closed ideal is $F$-closed, it again follows from parts (iii) and (i) of Lemma 7.4 that each primary component in this decomposition is $F$-closed.

By Lemmas 5.1 and 5.5, for each $i=1, \ldots, t$, the sequence

$$
\left(\left(\left(\left(\mathfrak{a}+\mathfrak{p}_{i}^{h}\right)^{\left[p^{n}\right]}\right)^{*}\right)^{e_{i} c_{i}}\right)_{n \in \mathbb{N}_{0}}
$$

is an $f$-sequence of $\mathfrak{p}_{i}$-primary ideals. It follows from Theorem 6.4 that the ideal of $R^{\infty}$ to which the $f$-sequence $\left(\left(\mathfrak{a}^{\left[p^{n}\right]}\right)^{*}\right)_{n \in \mathbb{N}_{0}}$ corresponds has a primary decomposition; all the remaining claims in part (ii) are now clear.

(iii) Let $c$ be a locally stable $p^{m_{0}}$-weak test element for $R$.

Arguments similar to those used in the above proof of part (ii) will show that

$$
\left(\mathfrak{a}^{\left[p^{n}\right]}\right)^{*} \subseteq \bigcap_{i=1}^{t}\left(\left(\left(\left(\mathfrak{a}+\mathfrak{p}_{i}^{h}\right)^{\left[p^{n}\right]}\right)^{e_{i}}\right)^{*}\right)^{c_{i}}
$$

and that $\left(\left(\left(\left(\mathfrak{a}+\mathfrak{p}_{i}^{h}\right)^{\left[p^{n}\right]}\right)^{e_{i}}\right)^{*}\right)^{c_{i}}$ is $\mathfrak{p}_{i}$-primary for each $i=1, \ldots, t$.

Now let $r \in \bigcap_{i=1}^{t}\left(\left(\left(\left(\mathfrak{a}+\mathfrak{p}_{i}^{h}\right)^{\left[p^{n}\right]}\right)^{e_{i}}\right)^{*}\right)^{c_{i}}$ and $j \in\{1, \ldots, t\}$. Then we have that $r / 1 \in\left(\left(\left(\mathfrak{a}+\mathfrak{p}_{j}^{h}\right)^{\left[p^{n}\right]}\right)^{e_{j}}\right)^{*}$; since $c / 1$ is a $p^{m_{0}}$-weak test element for $R_{\mathfrak{p}_{j}}$, we see that, for all $m \geq m_{0}$,

$$
\begin{aligned}
c r^{p^{m}} / 1 \in\left(\left(\left(\mathfrak{a}+\mathfrak{p}_{j}^{h}\right)^{\left[p^{n}\right]}\right)^{e_{j}}\right)^{\left[p^{m}\right]} & =\left(\left(\mathfrak{a}+\mathfrak{p}_{j}^{h}\right)^{\left[p^{m+n}\right]}\right)^{e_{j}}=\left(\mathfrak{a}^{\left[p^{m+n}\right]}+\mathfrak{p}_{j}^{h\left[p^{m+n}\right]}\right)^{e_{j}} \\
& \subseteq\left(\mathfrak{a}_{m+n}+\mathfrak{p}_{j}^{h\left[p^{m+n}\right]}\right)^{e_{j}} \subseteq\left(\left(\mathfrak{a}_{m+n}+\mathfrak{p}_{j}^{h\left[p^{m+n}\right]}\right)^{F}\right)^{e_{j}} .
\end{aligned}
$$

Therefore $c r^{p^{m}} \in\left(\left(\mathfrak{a}_{n+m}+\mathfrak{p}_{j}^{h\left[p^{n+m}\right]}\right)^{F}\right)^{e_{j} c_{j}}$ for all $m \geq m_{0}$. One can now use part (i) once again to deduce that $c r^{p^{m}} \in \mathfrak{a}_{n+m} \subseteq\left(\mathfrak{a}^{\left[p^{n+m}\right]}\right)^{*}$ for all $m \geq m_{0}$, and proceed as in the proof of part (ii) to conclude that $r \in\left(\mathfrak{a}^{\left[p^{n}\right]}\right)^{*}$. 
Thus

$$
\left(\mathfrak{a}^{\left[p^{n}\right]}\right)^{*}=\bigcap_{i=1}^{t}\left(\left(\left(\left(\mathfrak{a}+\mathfrak{p}_{i}^{h}\right)^{\left[p^{n}\right]}\right)^{e_{i}}\right)^{*}\right)^{c_{i}}
$$

is a primary decomposition, where $\left(\left(\left(\left(\mathfrak{a}+\mathfrak{p}_{i}^{h}\right)^{\left[p^{n}\right]}\right)^{e_{i}}\right)^{*}\right)^{c_{i}}$ is $\mathfrak{p}_{i}$-primary for $i=$ $1, \ldots, t$, and this time one can use Lemma 7.4 ii) to see that $\left.\left(\left(\left(\mathfrak{a}+\mathfrak{p}_{i}^{h}\right)^{\left[p^{n}\right]}\right)^{e_{i}}\right)^{*}\right)^{c_{i}}$ is tightly closed (for each $i=1, \ldots, t$ ).

The next theorem draws together results that now follow, in the presence of additional hypotheses, from the original hypothesis of Smith and Swanson that the Frobenius powers of the proper ideal $\mathfrak{a}$ of $R$ have linear growth of primary decompositions.

7.14. Theorem. Suppose that the Frobenius powers of the proper ideal $\mathfrak{a}$ of $R$ have linear growth of primary decompositions, and that $\bigcup_{n \in \mathbb{N}_{0}}$ ass $\mathfrak{a}^{\left[p^{n}\right]}$ is finite. Then

(i) the ideal $\mathfrak{a} R^{\infty}$ of $R^{\infty}$ to which the $f$-sequence $\left(\left(\mathfrak{a}^{\left[p^{n}\right]}\right)^{F}\right)_{n \in \mathbb{N}_{0}}$ corresponds has a primary decomposition;

(ii) the $f$-sequence $\left(\left(\mathfrak{a}^{\left[p^{n}\right]}\right)^{F}\right)_{n \in \mathbb{N}_{0}}$ has linear growth of primary decompositions;

(iii) $\bigcup_{n \in \mathbb{N}_{0}} \operatorname{ass}\left(\mathfrak{a}^{\left[p^{n}\right]}\right)^{F} \subseteq \bigcup_{n \in \mathbb{N}_{0}}$ ass $\mathfrak{a}^{\left[p^{n}\right]}$, and so the set $\bigcup_{n \in \mathbb{N}_{0}} \operatorname{ass}\left(\mathfrak{a}^{\left[p^{n}\right]}\right)^{F}$ is finite.

Furthermore, if $R$ has a $p^{m_{0}}$-weak test element, for some $m_{0} \in \mathbb{N}_{0}$, then, in addition,

(iv) $\mathfrak{a}^{*} S^{-1} R=\left(\mathfrak{a} S^{-1} R\right)^{*}$ for every multiplicatively closed subset $S$ of $R$;

(v) the ideal of $R^{\infty}$ to which the f-sequence $\left(\left(\mathfrak{a}^{\left[p^{n}\right]}\right)^{*}\right)_{n \in \mathbb{N}_{0}}$ corresponds has a primary decomposition, so that the $f$-sequence $\left(\left(\mathfrak{a}^{\left[p^{n}\right]}\right)^{*}\right)_{n \in \mathbb{N}_{0}}$ has linear growth of primary decompositions; and

(vi) $\bigcup_{n \in \mathbb{N}_{0}}$ ass $\left(\mathfrak{a}^{\left[p^{n}\right]}\right)^{*} \subseteq \bigcup_{n \in \mathbb{N}_{0}} \operatorname{ass}\left(\mathfrak{a}^{\left[p^{n}\right]}\right)^{F}$, so that the set $\bigcup_{n \in \mathbb{N}_{0}} \operatorname{ass}\left(\mathfrak{a}^{\left[p^{n}\right]}\right)^{*}$ is finite.

Proof. The claims in parts (i), (ii) and (iii) follow from Theorem 7.5. Since $\mathfrak{a}^{\left[p^{n}\right]} \subseteq$ $\left(\mathfrak{a}^{\left[p^{n}\right]}\right)^{F} \subseteq\left(\mathfrak{a}^{\left[p^{n}\right]}\right)^{*}$ for all $n \in \mathbb{N}_{0}$, all the other claims therefore follow from Theorems 7.6(ii) and 7.13(ii).

We end this section by drawing together in one theorem the conclusions of this section which together provide a strategy for attempting to show, for a given proper ideal $\mathfrak{a}$ of $R$, that tight closure commutes with localization at an arbitrary multiplicatively closed subset of $R$ and that $\bigcup_{n \in \mathbb{N}_{0}}$ ass $\left(\mathfrak{a}^{\left[p^{n}\right]}\right)^{*}$ is finite.

7.15. Theorem. Let $\mathfrak{a}$ be a proper ideal of $R$. Suppose that $\left(\mathfrak{a}_{n}\right)_{n \in \mathbb{N}_{0}}$ is an $f$ sequence of ideals of $R$ such that $\mathfrak{a}^{\left[p^{n}\right]} \subseteq \mathfrak{a}_{n} \subseteq\left(\mathfrak{a}^{\left[p^{n}\right]}\right)^{*}$ for all $n \in \mathbb{N}_{0}$. Suppose that the ideal $\mathfrak{A}$ of $R^{\infty}$ to which $\left(\mathfrak{a}_{n}\right)_{n \in \mathbb{N}_{0}}$ corresponds has a primary decomposition. Then

(i) the $f$-sequence $\left(\mathfrak{a}_{n}\right)_{n \in \mathbb{N}_{0}}$ has linear growth of primary decompositions;

(ii) the set $\bigcup_{n \in \mathbb{N}_{0}}$ ass $\mathfrak{a}_{n}$ is finite.

Furthermore, if $R$ has a $p^{m_{0}}$-weak test element, for some $m_{0} \in \mathbb{N}_{0}$, then, in addition,

(iii) $\mathfrak{a}^{*} S^{-1} R=\left(\mathfrak{a} S^{-1} R\right)^{*}$ for every multiplicatively closed subset $S$ of $R$; 
(iv) the ideal of $R^{\infty}$ to which the $f$-sequence $\left(\left(\mathfrak{a}^{\left[p^{n}\right]}\right)^{*}\right)_{n \in \mathbb{N}_{0}}$ corresponds has a primary decomposition, so that the $f$-sequence $\left(\left(\mathfrak{a}^{\left[p^{n}\right]}\right)^{*}\right)_{n \in \mathbb{N}_{0}}$ has linear growth of primary decompositions; and

(v) $\bigcup_{n \in \mathbb{N}_{0}}$ ass $\left(\mathfrak{a}^{\left[p^{n}\right]}\right)^{*} \subseteq \bigcup_{n \in \mathbb{N}_{0}}$ ass $\mathfrak{a}_{n}$, so that the set $\bigcup_{n \in \mathbb{N}_{0}}$ ass $\left(\mathfrak{a}^{\left[p^{n}\right]}\right)^{*}$ is finite.

Proof. The claims in (i) and (ii) follow from Theorem 17.9. The claim in (iii) then follows from Theorem 17.6(ii), while those in (iv) and (v) follow from Theorem 7.13(ii).

\section{Applications of the strategy}

Throughout this section, $R$ will denote a commutative Noetherian ring of prime characteristic $p$. The aim of this section is to give several examples of choices of $(R$ and) the proper ideal $\mathfrak{a}$ of $R$ for which the set $\bigcup_{n \in \mathbb{N}_{0}} \operatorname{ass}\left(\mathfrak{a}^{\left[p^{n}\right]}\right)^{*}$ is a finite set. We use either the strategy of Theorem 7.14 or that of Theorem 7.15, but in most of the examples presented below, it is already known that $\mathfrak{a}^{*} S^{-1} R=\left(\mathfrak{a} S^{-1} R\right)^{*}$ for every multiplicatively closed subset $S$ of $R$. Our first application uses Theorem 7.14.

8.1. Proposition. Let $\mathfrak{a}$ be a proper ideal of $R$ such that, for every $n \in \mathbb{N}_{0}$, all the associated primes of $\mathfrak{a}^{\left[p^{n}\right]}$ are minimal. Then

(i) the Frobenius powers of $\mathfrak{a}$ have linear growth of primary decompositions;

(ii) the ideal $\mathfrak{a} R^{\infty}$ of $R^{\infty}$ has a primary decomposition;

(iii) the $f$-sequence $\left(\left(\mathfrak{a}^{\left[p^{n}\right]}\right)^{F}\right)_{n \in \mathbb{N}_{0}}$ has linear growth of primary decompositions;

(iv) $\bigcup_{n \in \mathbb{N}_{0}}$ ass $\left(\mathfrak{a}^{\left[p^{n}\right]}\right)^{F}$ is equal to the set of minimal primes of $\mathfrak{a}$.

Furthermore, if $R$ has a $p^{m_{0}}$-weak test element, for some $m_{0} \in \mathbb{N}_{0}$, then, in addition,

(v) $\mathfrak{a}^{*} S^{-1} R=\left(\mathfrak{a} S^{-1} R\right)^{*}$ for every multiplicatively closed subset $S$ of $R$;

(vi) the ideal of $R^{\infty}$ to which the $f$-sequence $\left(\left(\mathfrak{a}^{\left[p^{n}\right]}\right)^{*}\right)_{n \in \mathbb{N}_{0}}$ corresponds has a primary decomposition, and so the $f$-sequence $\left(\left(\mathfrak{a}^{\left[p^{n}\right]}\right)^{*}\right)_{n \in \mathbb{N}_{0}}$ has linear growth of primary decompositions; and

(vii) the set $\bigcup_{n \in \mathbb{N}_{0}}$ ass $\left(\mathfrak{a}^{\left[p^{n}\right]}\right)^{*}$ is (finite and) equal to the set of minimal primes of $\mathfrak{a}$.

Proof. Let $\mathfrak{p}_{1}, \ldots, \mathfrak{p}_{t}$ be the minimal prime ideals of $\mathfrak{a}$. For each $i=1, \ldots, t$, let $e_{i}$ and $c_{i}$ stand for extension and contraction with respect to the natural ring homomorphism $R \longrightarrow R_{\mathfrak{p}_{i}}$.

Now $\mathfrak{a}^{\left[p^{n}\right]}=\bigcap_{i=1}^{t}\left(\mathfrak{a}^{\left[p^{n}\right]}\right)^{e_{i} c_{i}}$ is the unique minimal primary decomposition of $\mathfrak{a}^{\left[p^{n}\right]}$ (for each $n \in \mathbb{N}_{0}$ ). Choose $h \in \mathbb{N}$ such that $\mathfrak{p}_{i}^{h} \subseteq \mathfrak{a}^{e_{i} c_{i}}$ for all $i=1, \ldots, t$. Let $i \in\{1, \ldots, t\}$ and $r \in \mathfrak{p}_{i}^{h}$. Then there exists $s_{i} \in R \backslash \mathfrak{p}_{i}$ such that $s_{i} r \in \mathfrak{a}$, so that $s_{i}^{p^{n}} r^{p^{n}} \in \mathfrak{a}^{\left[p^{n}\right]}$ and $r^{p^{n}} \in\left(\mathfrak{a}^{\left[p^{n}\right]}\right)^{e_{i} c_{i}}$ (for all $n \in \mathbb{N}_{0}$ ). This shows that the Frobenius powers of $\mathfrak{a}$ have $h$-linear growth of primary decompositions.

The remaining claims follow from Theorem 7.14 (and the fact that a prime ideal of $R$ is tightly closed).

8.2. Example. If $R$ is a Cohen-Macaulay ring and $\mathfrak{a}$ is an ideal generated by a regular sequence $\left(r_{i}\right)_{i=1}^{k}$, then, for all $n \in \mathbb{N}_{0}$, the $n$-th Frobenius power $\mathfrak{a}^{\left[p^{n}\right]}$ is generated by the regular sequence $\left(r_{i}^{p^{n}}\right)_{i=1}^{k}$, and so is unmixed; thus the initial hypothesis of 8.1 is satisfied, and conclusions (i), (ii), (iii), (iv) of 8.1 hold. If, in 
addition, $R$ has a $p^{m_{0}}$-weak test element, for some $m_{0} \in \mathbb{N}_{0}$, then the other three conclusions of 8.1 also hold.

Some of the results in this section will concern situations where we can, in some sense, 'approximate' $R$ by a regular commutative Noetherian ring of characteristic $p$.

8.3. Remark. Suppose that $R$ is regular.

(i) As the Frobenius homomorphism $f: R \longrightarrow R$ is flat (see [11]), every ideal of $R$ is $F$-closed (and tightly closed).

(ii) It also follows from the fact that $f$ is flat that, if $\mathfrak{q}$ is a $\mathfrak{p}$-primary ideal of $R$, then so too is $\mathfrak{q}^{\left[p^{n}\right]}$ for all $n \in \mathbb{N}_{0}$, and that if $\mathfrak{a}=\mathfrak{q}_{1} \cap \cdots \cap \mathfrak{q}_{t}$ is a minimal primary decomposition of the proper ideal $\mathfrak{a}$ of $R$, then $\mathfrak{a}^{\left[p^{n}\right]}=$ $\mathfrak{q}_{1}^{\left[p^{n}\right]} \cap \cdots \cap \mathfrak{q}_{t}^{\left[p^{n}\right]}$ is also a minimal primary decomposition of $\mathfrak{a}^{\left[p^{n}\right]}$, for all $n \in \mathbb{N}_{0}$.

(iii) It is even the case that, if $\mathfrak{q}$ is a $\mathfrak{p}$-primary irreducible ideal of $R$, then $\mathfrak{q}^{\left[p^{n}\right]}$ is also irreducible, for each $n \in \mathbb{N}_{0}$. To see this, let $F_{R}$ denote the Frobenius functor $R^{\prime} \otimes_{R}(\cdot)$ on the category of $R$-modules and $R$-homomorphisms, where $R^{\prime}$ is $R$ considered as a left $R$-module in the usual manner and as a right $R$-module via the Frobenius homomorphism.

Since $E_{R}(R / \mathfrak{q}) \cong E_{R}(R / \mathfrak{p})$, it follows that $R / \mathfrak{q}^{[p]} \cong F(R / \mathfrak{q})$ is isomorphic to a submodule of $F\left(E_{R}(R / \mathfrak{p})\right)$; however, $F\left(E_{R}(R / \mathfrak{p})\right) \cong E_{R}(R / \mathfrak{p})$, by [8, Proposition 1.5], and so $E_{R}\left(R / \mathfrak{q}^{[p]}\right)$ must be isomorphic to an injective submodule of the indecomposable injective $R$-module $E_{R}(R / \mathfrak{p})$; therefore $E_{R}\left(R / \mathfrak{q}^{[p]}\right) \cong E_{R}(R / \mathfrak{p})$ and $\mathfrak{q}^{[p]}$ is irreducible.

8.4. Proposition. Suppose that $R$ is regular. Then each finitely generated proper ideal of $R^{\infty}$ has a primary decomposition.

Proof. Let $\mathfrak{A}$ be a finitely generated proper ideal in $R^{\infty}$. Let $\left(\mathfrak{a}_{n}\right)_{n \in \mathbb{N}_{0}}$ be the $f$ sequence corresponding to $\mathfrak{A}$. As every ideal in $R$ is $F$-closed (see 8.3(i)), it follows from Theorem 6.5 that there exists $k \in \mathbb{N}_{0}$ such that $\mathfrak{a}_{k+n}=\mathfrak{a}_{k}^{\left[p^{n}\right]}$ for all $n \in \mathbb{N}_{0}$.

Consider a minimal primary decomposition $\mathfrak{a}_{k}=\mathfrak{q}_{1, k} \cap \cdots \cap \mathfrak{q}_{t, k}$ of $\mathfrak{a}_{k}$. By [8.3](ii), for each $n \in \mathbb{N}_{0}$,

$$
\mathfrak{a}_{k+n}=\mathfrak{a}_{k}^{\left[p^{n}\right]}=\mathfrak{q}_{1, k}^{\left[p^{n}\right]} \cap \cdots \cap \mathfrak{q}_{t, k}^{\left[p^{n}\right]}
$$

is a minimal primary decomposition. Since every ideal of $R$ is $F$-closed, it follows from 4.1(iii) and4.2(iii) that there is, for each $j=1, \ldots, t$, an $f$-sequence $\left(\mathfrak{q}_{j, n}\right)_{n \in \mathbb{N}_{0}}$ of $\sqrt{\mathfrak{q}_{j, k}}$-primary ideals of $R$ with

$$
\mathfrak{q}_{j, n}= \begin{cases}\mathfrak{q}_{j, k}^{\left[p^{n-k}\right]} & \text { if } n \geq k, \\ f^{-(k-n)}\left(\mathfrak{q}_{j, k}\right) & \text { if } n<k .\end{cases}
$$

Note that $\mathfrak{a}_{n}=\mathfrak{q}_{1, n} \cap \cdots \cap \mathfrak{q}_{t, n}$ for all $n \in \mathbb{N}_{0}$. It now follows from Theorem 6.4 that $\mathfrak{A}$ has a primary decomposition.

We shall extend the result of Proposition 8.4 to a wider class of rings. Recall that a homomorphism of commutative rings $g: A \longrightarrow B$ is said to be pure if, for every $A$-module $M$, the map $M \otimes_{A} A \longrightarrow M \otimes_{A} B$ is injective. When this is the case, $g$ must be injective, we identify $A$ as a subring of $B$, and we say that $A$ is a pure subring of $B$; also, for each ideal $\mathfrak{a}$ of $A$, we have $\mathfrak{a} B \cap A=\mathfrak{a}$, so that $A$ is 
Noetherian if $B$ is. Note that if $A$ is a direct summand of $B$ as an $A$-module, then $A$ is a pure subring of $B$.

8.5. Lemma. Suppose that $R$ is a pure subring of the reduced commutative Noetherian ring $R^{\prime}$. Let $\mathfrak{A}$ be a proper ideal of $R^{\infty}$. Then

(i) $R^{\infty}$ is a pure subring of $R^{\prime \infty}$;

(ii) if $\mathfrak{A} R^{\prime \infty}$ has a primary decomposition as an ideal of $R^{\prime \infty}$, then $\mathfrak{A}$ has a primary decomposition in $R^{\infty}$; and

(iii) if $R^{\prime}$ is regular, then every finitely generated proper ideal of $R^{\infty}$ has a primary decomposition.

Proof. (i) It is straightforward to use [12, Theorem 7.13] to show that $R^{\infty}$ is a pure subring of $R^{\prime \infty}$.

(ii) This is now immediate, since it follows from part (i) and the comments preceding the statement of the lemma that $\mathfrak{A}=\mathfrak{A} R^{\prime \infty} \cap R^{\infty}$.

(iii) By Proposition 8.4 every finitely generated proper ideal of $R^{\prime \infty}$ has a primary decomposition. If $\mathfrak{A}$ is a finitely generated proper ideal of $R^{\infty}$, then $\mathfrak{A} R^{\prime \infty}$ is a finitely generated ideal of $R^{\prime \infty}$, and so the claim follows from part (ii).

8.6. Theorem. Let $R^{\prime \prime}$ be a pure subring of a regular (commutative Noetherian) ring of characteristic $p$, and assume that either $R$ is a purely inseparable extension ring of $R^{\prime \prime}$ or that $R^{\prime \prime}$ is a purely inseparable extension ring of $R$. Let $\mathfrak{a}$ be a proper ideal of $R$. Then

(i) every finitely generated proper ideal of $R^{\infty}$ has a primary decomposition;

(ii) the $f$-sequence $\left(\left(\mathfrak{a}^{\left[p^{n}\right]}\right)^{F}\right)_{n \in \mathbb{N}_{0}}$ has linear growth of primary decompositions;

(iii) the set $\bigcup_{n \in \mathbb{N}_{0}} \operatorname{ass}\left(\mathfrak{a}^{\left[p^{n}\right]}\right)^{F}$ is finite.

Furthermore, if $R$ has a $p^{m_{0}}$-weak test element, for some $m_{0} \in \mathbb{N}_{0}$, then, in addition,

(iv) $\mathfrak{a}^{*} S^{-1} R=\left(\mathfrak{a} S^{-1} R\right)^{*}$ for every multiplicatively closed subset $S$ of $R$;

(v) the ideal of $R^{\infty}$ to which the f-sequence $\left(\left(\mathfrak{a}^{\left[p^{n}\right]}\right)^{*}\right)_{n \in \mathbb{N}_{0}}$ corresponds has a primary decomposition, and so the $f$-sequence $\left(\left(\mathfrak{a}^{\left[p^{n}\right]}\right)^{*}\right)_{n \in \mathbb{N}_{0}}$ has linear growth of primary decompositions; and

(vi) $\bigcup_{n \in \mathbb{N}_{0}}$ ass $\left(\mathfrak{a}^{\left[p^{n}\right]}\right)^{*} \subseteq \bigcup_{n \in \mathbb{N}_{0}} \operatorname{ass}\left(\mathfrak{a}^{\left[p^{n}\right]}\right)^{F}$, so that the set $\bigcup_{n \in \mathbb{N}_{0}} \operatorname{ass}\left(\mathfrak{a}^{\left[p^{n}\right]}\right)^{*}$ is finite.

Proof. (i) Since $R^{\infty} \cong R^{\prime \prime \infty}$ (by 1.4) and $R^{\prime \prime}$ is Noetherian, we can assume that $R$ itself is a pure subring of a regular (commutative Noetherian) ring of characteristic $p$, and then the result follows from Lemma 8.5 because a regular ring is reduced.

All the other claims now follow from Theorem 7.15 , because the $f$-sequence $\left(\left(\mathfrak{a}^{\left[p^{n}\right]}\right)^{F}\right)_{n \in \mathbb{N}_{0}}$ corresponds to the finitely generated proper ideal $\mathfrak{a} R^{\infty}$ of $R^{\infty}$ (by Theorem [6.5), and so has a primary decomposition by part (i); note that $\mathfrak{a}^{\left[p^{n}\right]} \subseteq$ $\left(\mathfrak{a}^{\left[p^{n}\right]}\right)^{F} \subseteq\left(\mathfrak{a}^{\left[p^{n}\right]}\right)^{*}$ for all $n \in \mathbb{N}_{0}$.

Note that a ring $R$ satisfying the hypotheses of Theorem 8.6 need not have the property that every ideal is $F$-closed. As an example, consider $K\left[X^{2}, X^{3}\right] \subseteq K[X]$, where $K$ is a field of characteristic 2 and $X$ is an indeterminate. The regular ring $K[X]$ is a purely inseparable extension of $K\left[X^{2}, X^{3}\right]$. The ideal $\mathfrak{a}=\left(X^{2}\right) K\left[X^{2}, X^{3}\right]$ 
does not contain $X^{3}$, but

$$
\left(X^{3}\right)^{2}=X^{6} \in\left(X^{4}\right) K\left[X^{2}, X^{3}\right]=\left(\left(X^{2}\right) K\left[X^{2}, X^{3}\right]\right)^{[2]} .
$$

Therefore $\mathfrak{a}$ is not $F$-closed.

Our next two results are concerned with the case where $R$ is a domain and involve the absolute integral closure $R^{+}$of $R$; it should be noted that $R^{\infty}$ can be identified with the subring

$$
\left\{\sigma \in R^{+}: \text {there exists } n \in \mathbb{N}_{0} \text { such that } \sigma^{p^{n}} \in R\right\} .
$$

It is known that, if $R$ is an excellent regular local ring, then $R^{+}$is flat over $R$; see [7. Theorem 9.1] and [].

8.7. Proposition. Suppose that $R$ is a domain and that $R^{+}$is flat over $R$.

(i) Let $\mathfrak{q}$ be a $\mathfrak{p}$-primary ideal of $R$, and let $\mathfrak{P}$ be the prime ideal of $R^{\infty}$ corresponding to $\mathfrak{p}$. Then $\mathfrak{q} R^{+} \cap R^{\infty}$ is a $\mathfrak{P}$-primary ideal of $R^{\infty}$.

(ii) Let $\mathfrak{a}$ be a proper ideal of $R$, suppose that

$$
\mathfrak{a}=\mathfrak{q}_{1} \cap \cdots \cap \mathfrak{q}_{t}
$$

is a primary decomposition, where $\mathfrak{q}_{i}$ is $\mathfrak{p}_{i}$-primary for $i=1, \ldots, t$, and let $\mathfrak{P}_{1}, \ldots, \mathfrak{P}_{t}$ be the prime ideals of $R^{\infty}$ corresponding to $\mathfrak{p}_{1}, \ldots, \mathfrak{p}_{t}$, respectively. Then

$$
\mathfrak{a} R^{+} \cap R^{\infty}=\bigcap_{i=1}^{t}\left(\mathfrak{q}_{i} R^{+} \cap R^{\infty}\right)
$$

is a primary decomposition in $R^{\infty}$, where $\mathfrak{q}_{i} R^{+} \cap R^{\infty}$ is $\mathfrak{P}_{i}$-primary for $i=$ $1, \ldots, t$.

Proof. (i) Let $\rho \in R^{\infty}$. Then $\rho=r^{1 / p^{n}}$ for some $r \in R$ and $n \in \mathbb{N}_{0}$.

If $\rho \notin \mathfrak{P}$, then $r \notin \mathfrak{p}$ and multiplication by $r$ provides a monomorphism of the $R$-module $R / \mathfrak{q}$. Since $R^{+}$is flat over $R$, it follows that multiplication by $r$ provides a monomorphism of the $R$-module $(R / \mathfrak{q}) \otimes_{R} R^{+}$; the latter module is isomorphic to $R^{+} / \mathfrak{q} R^{+}$, and this has a submodule isomorphic to $R^{\infty} / \mathfrak{q} R^{+} \cap R^{\infty}$. Hence multiplication by $r$ provides a monomorphism of $R^{\infty} / \mathfrak{q} R^{+} \cap R^{\infty}$, and so multiplication by $r^{1 / p^{n}}=\rho$ also provides a monomorphism of this $R^{\infty}$-module.

On the other hand, if $\rho \in \mathfrak{P}$, then $r \in \mathfrak{p}$, and some power of $r$ belongs to $\mathfrak{q}$; hence some power of $r^{1 / p^{n}}=\rho$ belongs to $\mathfrak{q} R^{+} \cap R^{\infty}$. It follows that $\mathfrak{q} R^{+} \cap R^{\infty}$ is a $\mathfrak{P}$-primary ideal of $R^{\infty}$.

(ii) Since $R^{+}$is flat over $R$, we have $\mathfrak{a} R^{+}=\bigcap_{i=1}^{t} \mathfrak{q}_{i} R^{+}$, and so $\mathfrak{a} R^{+} \cap R^{\infty}=$ $\bigcap_{i=1}^{t}\left(\mathfrak{q}_{i} R^{+} \cap R^{\infty}\right)$. Also, it follows from part (i) that $\mathfrak{q}_{i} R^{+} \cap R^{\infty}$ is $\mathfrak{P}_{i}$-primary for all $i=1, \ldots, t$.

8.8. Corollary. Suppose that $R$ is a subring of an excellent regular local ring $R^{\prime}$ and that $R^{\prime}$ is integral over $R$. Let $\mathfrak{a}$ be a proper ideal of $R$. Then

(i) the ideal of $R^{\infty}$ to which the $f$-sequence $\left(\left(\mathfrak{a}^{\left[p^{n}\right]}\right)^{+}\right)_{n \in \mathbb{N}_{0}}$ corresponds has a primary decomposition;

(ii) the $f$-sequence $\left(\left(\mathfrak{a}^{\left[p^{n}\right]}\right)^{+}\right)_{n \in \mathbb{N}_{0}}$ has linear growth of primary decompositions;

(iii) the set $\bigcup_{n \in \mathbb{N}_{0}} \operatorname{ass}\left(\mathfrak{a}^{\left[p^{n}\right]}\right)^{+}$is finite. 
Furthermore, if $R$ has a $p^{m_{0}}$-weak test element, for some $m_{0} \in \mathbb{N}_{0}$, then, in addition,

(iv) $\mathfrak{a}^{*} S^{-1} R=\left(\mathfrak{a} S^{-1} R\right)^{*}$ for every multiplicatively closed subset $S$ of $R$;

(v) the ideal of $R^{\infty}$ to which the f-sequence $\left(\left(\mathfrak{a}^{\left[p^{n}\right]}\right)^{*}\right)_{n \in \mathbb{N}_{0}}$ corresponds has a primary decomposition, and so the $f$-sequence $\left(\left(\mathfrak{a}^{\left[p^{n}\right]}\right)^{*}\right)_{n \in \mathbb{N}_{0}}$ has linear growth of primary decompositions; and

(vi) $\bigcup_{n \in \mathbb{N}_{0}}$ ass $\left(\mathfrak{a}^{\left[p^{n}\right]}\right)^{*} \subseteq \bigcup_{n \in \mathbb{N}_{0}} \operatorname{ass}\left(\mathfrak{a}^{\left[p^{n}\right]}\right)^{+}$, so that the set $\bigcup_{n \in \mathbb{N}_{0}} \operatorname{ass}\left(\mathfrak{a}^{\left[p^{n}\right]}\right)^{*}$ is

Note. Actually, the conclusion of part (iv) can be established easily, even without the existence of a $p^{m_{0}}$-weak test element, by a combination of arguments in the proof of K. E. Smith [14, Lemma 2] and in Huneke [7, p. 15].

Proof. (i) We have $R^{\prime+}=R^{+}$; since $R^{\prime+}$ is flat over $R^{\prime}$ (see [7, Proposition 9.1]), it follows from Proposition 8.7 that the ideal

$$
\mathfrak{a} R^{+} \cap R^{\prime \infty}=\mathfrak{a} R^{\prime+} \cap R^{\prime \infty}=\left(\mathfrak{a} R^{\prime}\right) R^{\prime+} \cap R^{\prime \infty}
$$

of $R^{\prime \infty}$ has a primary decomposition. Now $R^{\infty}$ is a subring of $R^{\prime \infty}$, and so it follows that $\mathfrak{a} R^{+} \cap R^{\infty}$, which is the contraction of $\mathfrak{a} R^{+} \cap R^{\prime \infty}$ to $R^{\infty}$, has a primary decomposition. However, by Lemma 5.2 the ideal $\mathfrak{a} R^{+} \cap R^{\infty}$ of $R^{\infty}$ corresponds to the $f$-sequence $\left(\left(\mathfrak{a}^{\left[p^{n}\right]}\right)^{+}\right)_{n \in \mathbb{N}_{0}}$.

All the remaining claims now follow from Theorem 7.15 because $\mathfrak{a}^{\left[p^{n}\right]} \subseteq\left(\mathfrak{a}^{\left[p^{n}\right]}\right)^{+}$ $\subseteq\left(\mathfrak{a}^{\left[p^{n}\right]}\right)^{*}$ for all $n \in \mathbb{N}_{0}$.

The next theorem is one of the main results of this section. It should be noted that many cases of the result of 8.9 (iv) about localization of tight closure follow from the result of I. M. Aberbach, Hochster and Huneke in [1, Theorem (6.9)]; however, the proof below is very different from theirs. All the other results of 8.9 are believed to be new.

8.9. Theorem. Suppose that $R$ is equidimensional [12 p. 250] and integral over a regular excellent subring $A$, and let $\mathfrak{a}$ be an ideal of $R$ which is the extension of a proper ideal $\mathfrak{b}$ of $A$, that is, $\mathfrak{a}=\mathfrak{b} R$. Then

(i) the ideal of $R^{\infty}$ to which the $f$-sequence $\left(\left(\mathfrak{a}^{\left[p^{n}\right]}\right)^{+}\right)_{n \in \mathbb{N}_{0}}$ (see 5.3 and 5.4) corresponds has a primary decomposition;

(ii) the $f$-sequence $\left(\left(\mathfrak{a}^{\left[p^{n}\right]}\right)^{+}\right)_{n \in \mathbb{N}_{0}}$ has linear growth of primary decompositions;

(iii) the set $\bigcup_{n \in \mathbb{N}_{0}} \operatorname{ass}\left(\mathfrak{a}^{\left[p^{n}\right]}\right)^{+}$is finite.

Furthermore, if $R$ has a $p^{m_{0}}$-weak test element, for some $m_{0} \in \mathbb{N}_{0}$, then, in addition,

(iv) (see Aberbach-Hochster-Huneke [1, Theorem (6.9)]) $\mathfrak{a}^{*} S^{-1} R=\left(\mathfrak{a} S^{-1} R\right)^{*}$ for every multiplicatively closed subset $S$ of $R$;

(v) the ideal of $R^{\infty}$ to which the f-sequence $\left(\left(\mathfrak{a}^{\left[p^{n}\right]}\right)^{*}\right)_{n \in \mathbb{N}_{0}}$ corresponds has a primary decomposition, and so the $f$-sequence $\left(\left(\mathfrak{a}^{\left[p^{n}\right]}\right)^{*}\right)_{n \in \mathbb{N}_{0}}$ has linear growth of primary decompositions; and (vi) $\bigcup_{n \in \mathbb{N}_{0}} \operatorname{ass}\left(\mathfrak{a}^{\left[p^{n}\right]}\right)^{*} \subseteq \bigcup_{n \in \mathbb{N}_{0}} \operatorname{ass}\left(\mathfrak{a}^{\left[p^{n}\right]}\right)^{+}$, so that the set $\bigcup_{n \in \mathbb{N}_{0}} \operatorname{ass}\left(\mathfrak{a}^{\left[p^{n}\right]}\right)^{*}$ is

Proof. (i) Suppose that there are $h$ minimal prime ideals $\mathfrak{p}_{1}, \ldots, \mathfrak{p}_{h}$ of $R$. Let $i \in\{1, \ldots, h\}$. Since $R$ is equidimensional, we have

$$
\operatorname{dim} A / A \cap \mathfrak{p}_{i}=\operatorname{dim} R / \mathfrak{p}_{i}=\operatorname{dim} R=\operatorname{dim} A
$$


and so $A \cap \mathfrak{p}_{i}$ is a minimal prime ideal of $A$. Therefore $A / A \cap \mathfrak{p}_{i}$ is a direct factor of $A$, and so is itself a regular excellent domain, and the extension integral domain $R / \mathfrak{p}_{i}$ is integral over it. One can now use Lemma 5.4 to see that it is sufficient to establish part (i) under the additional hypothesis that $R$ is a domain, and so it is assumed that that is the case for the remainder of the proof of part (i). However, in that case, since the class of excellent rings is closed under localization, it follows from [7, Theorem 9.1] that $A^{+}$is flat over $A$. Note that $A^{+}=R^{+}$, and when we identify $R^{\infty}$ and $A^{\infty}$ with subrings of $A^{+}=R^{+}$in the natural way, we have $A^{\infty} \subseteq R^{\infty} \subseteq A^{+}=R^{+}$.

Let $\mathfrak{q}$ be a $\mathfrak{p}$-primary ideal of $A$. We next show that $(\mathfrak{q} R) R^{+} \cap R$ has no embedded associated prime ideal. Note that $(\mathfrak{q} R) R^{+}=\mathfrak{q} A^{+}$. Hence $\left((\mathfrak{q} R) R^{+} \cap R\right) \cap A=$ $\mathfrak{q} A^{+} \cap A=\mathfrak{q}^{+}=\mathfrak{q}$ since $A$ is regular, so that every ideal of $A$ is tightly closed. Suppose that $\mathfrak{r} \in \operatorname{Spec}(R)$ is an embedded prime ideal of $(\mathfrak{q} R) R^{+} \cap R$, and seek a contradiction. Then there exists a minimal prime ideal $\mathfrak{s} \in \operatorname{ass}_{R}\left((\mathfrak{q} R) R^{+} \cap R\right)$ with $\mathfrak{s} \subset \mathfrak{r}$ (the symbol ' $\subset$ ' is reserved to denote strict inclusion). Since $R$ is integral over $A$, it follows that $\mathfrak{p} \subseteq \mathfrak{s} \cap A \subset \mathfrak{r} \cap A$. This means that there exists $a \in \mathfrak{r} \cap A$ such that multiplication by $a$ provides a monomorphism of $A / \mathfrak{q}$. Since $A^{+}$is flat over $A$, multiplication by a provides a monomorphism of $A^{+} / \mathfrak{q} A^{+}=R^{+} /(\mathfrak{q} R) R^{+}$; hence multiplication by a provides a monomorphism of $R /(\mathfrak{q} R) R^{+} \cap R$. But this is a contradiction, because $\mathfrak{r}$ consists of zerodivisors on $R /(\mathfrak{q} R) R^{+} \cap R$. This shows that $(\mathfrak{q} R)^{+}=(\mathfrak{q} R) R^{+} \cap R$ has no embedded prime.

Furthermore, since $A$ is regular, the Frobenius powers of the p-primary ideal $\mathfrak{q}$ of $A$ are again $\mathfrak{p}$-primary (see 8.3 (ii)), so that, by the above paragraph,

$$
\left(\mathfrak{q}^{\left[p^{n}\right]} R\right) R^{+} \cap R=\left((\mathfrak{q} R)^{\left[p^{n}\right]}\right)^{+}
$$

has no embedded prime (for each $n \in \mathbb{N}_{0}$ ). It now follows from 7.12 that the $f$ sequence $\left(\left((\mathfrak{q} R)^{\left[p^{n}\right]}\right)^{+}\right)_{n \in \mathbb{N}_{0}}$ has linear growth of primary decompositions, and the ideal of $R^{\infty}$ to which it corresponds has a primary decomposition.

Now let $\mathfrak{b}=\mathfrak{q}_{1} \cap \cdots \cap \mathfrak{q}_{t}$, where $\mathfrak{q}_{i}$ is $\mathfrak{p}_{i}$-primary for $i=1, \ldots, t$, be a minimal primary decomposition of $\mathfrak{b}$ in $A$. Then, by 8.3(ii), for each $n \in \mathbb{N}_{0}$,

$$
\mathfrak{b}^{\left[p^{n}\right]}=\mathfrak{q}_{1}^{\left[p^{n}\right]} \cap \cdots \cap \mathfrak{q}_{t}^{\left[p^{n}\right]}, \quad \text { where } \mathfrak{q}_{i}^{\left[p^{n}\right]} \text { is } \mathfrak{p}_{i} \text {-primary for } i=1, \ldots, t,
$$

is again a minimal primary decomposition. Since $A^{+}$is flat over $A$, we have, again for each $n \in \mathbb{N}_{0}$, that $\mathfrak{b}^{\left[p^{n}\right]} A^{+}=\bigcap_{i=1}^{t} \mathfrak{q}_{i}^{\left[p^{n}\right]} A^{+}$, that is, $(\mathfrak{b} R)^{\left[p^{n}\right]} R^{+}=$ $\bigcap_{i=1}^{t}\left(\mathfrak{q}_{i} R\right)^{\left[p^{n}\right]} R^{+}$. Now contract back to $R$, and recall that $\mathfrak{a}=\mathfrak{b} R$ : one obtains that

$$
\left(\mathfrak{a}^{\left[p^{n}\right]}\right)^{+}=\bigcap_{i=1}^{t}\left(\left(\mathfrak{q}_{i} R\right)^{\left[p^{n}\right]}\right)^{+} \quad \text { for all } n \in \mathbb{N}_{0} .
$$

By the immediately preceding paragraph, the ideal $\mathfrak{Q}_{i}$ of $R^{\infty}$ to which the $f$ sequence $\left(\left(\left(\mathfrak{q}_{i} R\right)^{\left[p^{n}\right]}\right)^{+}\right)_{n \in \mathbb{N}_{0}}$ corresponds has a primary decomposition (for each $i=1, \ldots, t)$. Therefore $\bigcap_{i=1}^{t} \mathfrak{Q}_{i}$ has a primary decomposition; however, by 6.1 $(\mathrm{v})$, the ideal $\bigcap_{i=1}^{t} \mathfrak{Q}_{i}$ corresponds to the $f$-sequence $\left(\left(\mathfrak{a}^{\left[p^{n}\right]}\right)^{+}\right)_{n \in \mathbb{N}_{0}}$. This completes the proof of part (i).

All the remaining claims now follow from Theorem[7.15 because $\mathfrak{a}^{\left[p^{n}\right]} \subseteq\left(\mathfrak{a}^{\left[p^{n}\right]}\right)^{+}$ $\subseteq\left(\mathfrak{a}^{\left[p^{n}\right]}\right)^{*}$ for all $n \in \mathbb{N}_{0}$, by Lemma 5.4 (i).

8.10. Corollary. Assume that $R$ is an equidimensional complete local ring of dimension $d$, and let $x_{1}, \ldots, x_{d}$ form a system of parameters for $R$. Let $\mathfrak{a}$ denote a 
proper ideal of $R$ generated by 'polynomials' or 'formal power series' in $x_{1}, \ldots, x_{d}$ with coefficients in the prime subfield of $R$ (or even in a coefficient field $K$ of $R$ ). Then

(i) the ideal of $R^{\infty}$ to which the $f$-sequence $\left(\left(\mathfrak{a}^{\left[p^{n}\right]}\right)^{+}\right)_{n \in \mathbb{N}_{0}}$ corresponds has a primary decomposition;

(ii) the $f$-sequence $\left(\left(\mathfrak{a}^{\left[p^{n}\right]}\right)^{+}\right)_{n \in \mathbb{N}_{0}}$ has linear growth of primary decompositions;

(iii) the set $\bigcup_{n \in \mathbb{N}_{0}} \operatorname{ass}\left(\mathfrak{a}^{\left[p^{n}\right]}\right)^{+}$is finite;

(iv) $\mathfrak{a}^{*} S^{-1} R=\left(\mathfrak{a} S^{-1} R\right)^{*}$ for every multiplicatively closed subset $S$ of $R$;

(v) the ideal of $R^{\infty}$ to which the $f$-sequence $\left(\left(\mathfrak{a}^{\left[p^{n}\right]}\right)^{*}\right)_{n \in \mathbb{N}_{0}}$ corresponds has a primary decomposition, and so the $f$-sequence $\left(\left(\mathfrak{a}^{\left[p^{n}\right]}\right)^{*}\right)_{n \in \mathbb{N}_{0}}$ has linear growth of primary decompositions; and

(vi) $\bigcup_{n \in \mathbb{N}_{0}} \operatorname{ass}\left(\mathfrak{a}^{\left[p^{n}\right]}\right)^{*} \subseteq \bigcup_{n \in \mathbb{N}_{0}}$ ass $\left(\mathfrak{a}^{\left[p^{n}\right]}\right)^{+}$, so that the set $\bigcup_{n \in \mathbb{N}_{0}} \operatorname{ass}\left(\mathfrak{a}^{\left[p^{n}\right]}\right)^{*}$ is finite.

Proof. By Cohen's structure theorems for complete local rings, there exists a coefficient field $K$ of $R$, and $R$ is a finite module over its complete regular local subring $A:=K\left[\left[x_{1}, \ldots, x_{d}\right]\right]$; see [12, Theorem 29.4], for example. Now a complete local ring is excellent, and so $A$ is an excellent regular local ring, and $R$ has a $p^{m_{0}}$-weak test element, for some $m_{0} \in \mathbb{N}_{0}$, by Hochster-Huneke [5, Theorem (6.1)(b)]. All the claims therefore follow from Theorem 8.9 .

The final corollary gives a partial extension of the results of Corollary 8.10(iv)(vi) to an equidimensional excellent local ring.

8.11. Corollary. Assume that $R$ is an equidimensional excellent local ring of dimension $d$, and let $x_{1}, \ldots, x_{d}$ form a system of parameters for $R$. Let $\mathfrak{a}$ denote a proper ideal of $R$ generated by 'polynomials' in $x_{1}, \ldots, x_{d}$ with coefficients in the prime subfield of $R$. Then

(i) $\mathfrak{a}^{*} S^{-1} R=\left(\mathfrak{a} S^{-1} R\right)^{*}$ for every multiplicatively closed subset $S$ of $R$;

(ii) the ideal of $R^{\infty}$ to which the $f$-sequence $\left(\left(\mathfrak{a}^{\left[p^{n}\right]}\right)^{*}\right)_{n \in \mathbb{N}_{0}}$ corresponds has a primary decomposition, so that the $f$-sequence $\left(\left(\mathfrak{a}^{\left[p^{n}\right]}\right)^{*}\right)_{n \in \mathbb{N}_{0}}$ has linear growth of primary decompositions; and

(iii) the set $\bigcup_{n \in \mathbb{N}_{0}} \operatorname{ass}\left(\mathfrak{a}^{\left[p^{n}\right]}\right)^{*}$ is finite.

Proof. We first show that we can apply Corollary 8.10 to the completion $\widehat{R}$ of $R$, by showing that $\widehat{R}$ is also equidimensional. Let $\widehat{\mathfrak{p}}$ be a minimal prime ideal of $\widehat{R}$, and let $\mathfrak{p}:=\widehat{\mathfrak{p}} \cap R$. Then $\operatorname{dim} R / \mathfrak{p}=d$, and $\widehat{\mathfrak{p}} / \mathfrak{p} \widehat{R}$ is a minimal prime of the completion $\widehat{R} / \mathfrak{p} \widehat{R}$ of $R / \mathfrak{p}$. Since $R$ is universally catenary, it follows from a theorem of L. J. Ratliff (see [12, Theorem 31.7]) that

$$
\operatorname{dim} \widehat{R} / \widehat{\mathfrak{p}}=\operatorname{dim}(\widehat{R} / \mathfrak{p} \widehat{R}) /(\widehat{\mathfrak{p}} / \mathfrak{p} \widehat{R})=\operatorname{dim} \widehat{R} / \mathfrak{p} \widehat{R}=\operatorname{dim} R / \mathfrak{p}=\operatorname{dim} R=\operatorname{dim} \widehat{R} .
$$

Hence $\widehat{R}$ is equidimensional.

It now follows from Corollary 8.10 that the ideal of $\widehat{R}^{\infty}$ to which the $f$-sequence $\left(\left((\mathfrak{a} \widehat{R})^{\left[p^{n}\right]}\right)^{+}\right)_{n \in \mathbb{N}_{0}}$ (of ideals of $\left.\widehat{R}\right)$ corresponds has a primary decomposition. Hence, by 4.4, the ideal of $R^{\infty}$ to which the $f$-sequence $\left(\left((\mathfrak{a} \widehat{R})^{\left[p^{n}\right]}\right)^{+} \cap R\right)_{n \in \mathbb{N}_{0}}$ (of ideals of $R$ ) corresponds has a primary decomposition; note that

$$
\mathfrak{a}^{\left[p^{n}\right]} \subseteq\left((\mathfrak{a} \widehat{R})^{\left[p^{n}\right]}\right)^{+} \cap R \subseteq\left((\mathfrak{a} \widehat{R})^{\left[p^{n}\right]}\right)^{*} \cap R
$$


for all $n \in \mathbb{N}_{0}$. But $R$ has a completely stable $p^{m_{0}}$-weak test element, for some $m_{0} \in \mathbb{N}_{0}$, by Hochster-Huneke [5] Theorem (6.1)(b)], and it is easy to use this to see that $\left((\mathfrak{a} \widehat{R})^{\left[p^{n}\right]}\right)^{*} \cap R=\left(\mathfrak{a}^{\left[p^{n}\right]}\right)^{*}$ for all $n \in \mathbb{N}_{0}$. All the claims now follow from Theorem 7.15.

\section{REFERENCES}

1. I. M. Aberbach, M. Hochster and C. Huneke, Localization of tight closure and modules of finite phantom projective dimension, J. reine angew. Math. 434 (1993) 67-114. MR 94h:13005

2. M. J. Greenberg, Perfect closures of rings and schemes, Proc. Amer. Math. Soc. 16 (1965) 313-317. MR 32:7583

3. M. Hochster and C. Huneke, Tight closure, invariant theory and the Briançon-Skoda Theorem, J. Amer. Math. Soc. 3 (1990) 31-116. MR 91g:13010

4. M. Hochster and C. Huneke, Infinite integral extensions and big Cohen-Macaulay algebras, Annals of Math. 135 (1992) 53-89. MR 92m:13023

5. M. Hochster and C. Huneke, F-regularity, test elements, and smooth base change, Transactions Amer. Math. Soc. 346 (1994) 1-62. MR 95d:13007

6. M. Hochster and C. Huneke, Applications of the existence of big Cohen-Macaulay algebras, Advances in Math. 113 (1995) 45-117. MR 96d:13014

7. C. Huneke, Tight closure and its applications, Conference Board of the Mathematical Sciences Regional Conference Series in Mathematics 88, American Mathematical Society, Providence, 1996. MR 96m:13001

8. C. Huneke and R. Y. Sharp, Bass numbers of local cohomology modules, Transactions Amer. Math. Soc. 339 (1993) 765-779. MR 93m:13008

9. D. A. Jordan, Bijective extensions of injective ring endomorphisms, J. London Math. Soc. (2) 25 (1982) 435-448. MR 83j:16002

10. M. Katzman, Finiteness of $\bigcup_{e} \operatorname{Ass} F^{e}(M)$ and its connections to tight closure, Illinois J. of Math. 40 (1996) 330-337. MR 97i:13003

11. E. Kunz, Characterizations of regular local rings of characteristic p, Amer. J. Math. 91 (1969) 772-784. MR 40:5609

12. H. Matsumura, Commutative ring theory, Cambridge Studies in Advanced Mathematics 8, Cambridge University Press, 1986. MR 88h:13001

13. R. Y. Sharp, Injective modules and linear growth of primary decompositions, Proc. Amer. Math. Soc. 128 (2000) 717-722. MR 2000e:13004

14. K. E. Smith, Tight closure commutes with localization in binomial rings, Proc. Amer. Math. Soc. 129 (2001) 667-669. MR 2001f:13008

15. K. E. Smith and I. Swanson, Linear bounds on growth of associated primes, Comm. Algebra 25 (1997) 3071-3079. MR 98k:13003

16. I. Swanson, Powers of ideals. Primary decompositions, Artin-Rees lemma and regularity, Math. Ann. 307 (1997) 299-313. MR 97j:13005

Department of Pure Mathematics, University of Sheffield, Hicks Building, Sheffield S3 7RH, UNITED KINGDOM

E-mail address: R.Y.Sharp@sheffield.ac.uk

Department of Pure Mathematics, University of Sheffield, Hicks Building, Sheffield S3 7RH, United Kingdom

E-mail address: N.Nossem@sheffield.ac.uk 